\title{
The efficient inclusion of rotation-induced inertia effects in a shaft-blisk assembly model using zero-speed modes
}

DOI:

10.1016/j.jsv.2020.115357

Document Version

Accepted author manuscript

Link to publication record in Manchester Research Explorer

Citation for published version (APA):

Mohamed, M. E., \& Bonello, P. (2020). The efficient inclusion of rotation-induced inertia effects in a shaft-blisk assembly model using zero-speed modes. Journal of Sound and Vibration.

https://doi.org/10.1016/j.jsv.2020.115357

\section{Published in:}

Journal of Sound and Vibration

\section{Citing this paper}

Please note that where the full-text provided on Manchester Research Explorer is the Author Accepted Manuscript or Proof version this may differ from the final Published version. If citing, it is advised that you check and use the publisher's definitive version.

\section{General rights}

Copyright and moral rights for the publications made accessible in the Research Explorer are retained by the authors and/or other copyright owners and it is a condition of accessing publications that users recognise and abide by the legal requirements associated with these rights.

\section{Takedown policy}

If you believe that this document breaches copyright please refer to the University of Manchester's Takedown Procedures [http://man.ac.uk/04Y6Bo] or contact uml.scholarlycommunications@manchester.ac.uk providing relevant details, so we can investigate your claim.

\section{OPEN ACCESS}




\title{
The Efficient Inclusion of Rotation-Induced Inertia Effects in a Shaft- Blisk Assembly Model using Zero-Speed Modes
}

\author{
Mohamed Elsayed Mohamed ${ }^{\mathrm{a}, \mathrm{b}_{*}}$, Philip Bonello ${ }^{\mathrm{a}}$ \\ ${ }^{\mathrm{a}}$ School of Mechanical, Aerospace and Civil Engineering, University of Manchester, Manchester, \\ United Kingdom \\ bepartment of Mechanical Design and Production, Faculty of Engineering, Cairo University, Giza, \\ Egypt \\ *Corresponding author. \\ E-mail address: mohamed.mohamed-2@manchester.ac.uk (M. E. Mohamed)
}

\begin{abstract}
A successful design of rotating shaft-bladed disk ("blisk") assemblies requires the facility to reliably predict the resonance speeds, which must be avoided during operation to avoid failure. Rotation-induced inertia effects (centrifugal stiffening, spin softening, Coriolis forces and gyroscopic moments) can cause significant variation of the modal frequencies with speed. A review of the state-of-the-art highlights the need for a methodology that can efficiently include all rotation-induced inertia effects in a generic shaft-blisk system. The novel contribution of this paper is a methodology to include such effects in a generic shaftblisk system, using zero-speed finite element (FE) modal data, without the need for additional FE analysis at each speed, or the derivation of equations from first principles. This contribution is motivated by the need to upgrade an existing blisk simulator designed to generate blade tip timing (BTT) data for the development and validation of BTT algorithms. Rotational effects are added as discretised "external" excitations to the modal equations, which remain based on the zero-speed modes. The method is progressively validated on six examples, using results from the literature and commercial FE rotordynamics software, to demonstrate its accuracy and high efficiency of computation for both Campbell diagrams and forced response.
\end{abstract}




\section{Keywords}

Bladed Disk Assembly; Rotational Effects; Finite Element Analysis; Modal Analysis; Campbell Diagrams

\section{INTRODUCTION}

Shaft-blisk ("bladed disk") assemblies in turbomachinery often fail due to fatigue when they are exposed to excessive vibration in the resonant condition. To avoid such failure, it is of importance to accurately predict the vibration modes during the design stage. The deformations of the shaft, disk and blades are always to be coupled to some extent [1]. However, owing to the complexity of the problem, the majority of the early studies have been limited to analyses of individual elements, which is appropriate for cases where the fundamental natural frequency of the individual element is much lower than that of the other elements. The high speed, light weight, and large scale of modern turbomachinery have increased the importance of the higher critical speeds of such systems. This has consequently increased the need to study the effect of rotation-induced inertia effects on the vibration characteristics. These effects are: centrifugal stiffening, spin softening, Coriolis forces and gyroscopic moments [2]. In the absence of such effects, the natural frequencies of the shaftblisk system are independent of speed. Rotation-induced inertia effects result in the splitting of certain natural frequencies with increasing speed on the Campbell diagram (natural frequencies vs speed graph) [3].

The early analysis of blades was based on one-dimensional beam theory. Later on, rotationinduced inertia effects were included in the beam model [4]. For beams, these effects come from centrifugal and Coriolis forces. Such effects were neglected in some studies [5-8] and considered in others [9-11]. For shaft-blisk assemblies, the accurate modelling of rotation effects requires consideration of disk flexibility. Preliminary studies based on simple models have shown the possible effect of disk flexibility on the critical speeds of rotating solid shafts 
[12-15]. Crawley et al. [16] showed that the blade modes with one nodal diameter dynamically couple to the rigid body whirling motion of the shaft-disk system. Shahab and Thomas [17] showed that the transverse movement of the shaft can excite the backward or forward whirling of the shaft-blisk system.

In order to get an accurate prediction of the dynamic characteristics of turbomachinery, one ought to model a shaft-blisk system as an assembly, in which all the elements are dynamically coupled together [18]. Methods such as finite element (FEM), dynamic stiffness (DSM), assumed modes (AMM), and experimental methods, or combinations of these methods were used for modelling and analysis of rotating assemblies including the flexibility and dynamic couplings between different components [18-22].

In case of a single nodal diameter whirling mode of the blisk, a pitching force/moment is exerted on the shaft which couples the bending behaviour of the blisk to the shaft whirl through gyroscopic effect, resulting in forward and backward whirl modes [23]. The gyroscopic effects, rotary inertia, and shear deformation of the shaft can be ignored relative to those of the blisk at intermediate shaft speeds [23].

Some early studies, such as the one of Suyehiro, assumed that the gyroscopic load is acting on the mid-plane of a stationary disk [24]. Dopkin and Shoup [12] observed that the disk bending modes with one nodal diameter produce a net moment on the shaft and therefore coupled with the shaft flexural modes through gyroscopic effect. In their study, the shaft was modelled as lumped mass segments, while constant thickness plate elements (rings) and concentrated mass elements were used to model the disk. Many other studies considered the gyroscopic effects of one or more components such as $[1,2,25,26]$. A new dynamic model of a rotor/rigid disk/blade system was presented in 2015 by Ma et al. [2]. The model considered the gyroscopic effects of the disk and shaft, the Coriolis forces, centrifugal stiffening, and 
spin softening of the blades, in addition to both the lateral and torsional deformations of the shaft. The blades were modelled using Timoshenko beam elements. The equations of motion were derived by using Hamilton's principle and the assumed mode method. The analytical model was validated against both the finite element model by ANSYS FE software and experimentally. The centrifugal stiffening and spin softening effects were included in the ANSYS model stiffness matrix by using pre-stressed modal analysis which requires a separate analysis at each speed based on a static analysis.

The trend towards increased efficiency at higher speeds has led to larger blades and stronger stagger angles, which generate large centrifugal and Coriolis forces [26]. Some early studies ignored the Coriolis effects since they were limited to small motions or to beams that are rigid in bending except at disk connection [27]. Krupka and Baumanis [28] found that both shear deformation and rotary inertia should be included in the analysis, as they result in changes of about $4 \%$ to $8 \%$ in first and second natural frequencies at high speeds. Sisto et al. [29] showed with high accuracy that the Coriolis forces alter the stability of the blades.

A discrete system representation, at which the blade is divided into a number of segments has been employed in some studies [26, 29]. This facilitates the inclusion of Coriolis forces, shear and inertia effects. Xin and Wang found that the maximum frequency split due to Coriolis effects occurs for single nodal diameter modes [31]. A new test rig was developed in 2015 by Ruffini et al. [32] to study the Coriolis effects on blisk-shaft assemblies, and to provide validation data for the available numerical methods. The effect of Coriolis forces on the forced response of mistuned bladed-disk systems was studied by Kan et al. [33].

Rotating cantilever beam models have been used for turbomachinery blades. The vibration characteristics of rotating beams vary significantly with the increase in speed. One of the factors responsible for this variation is the centrifugal inertia force, which causes a significant 
stretch in the blades, resulting in a change in the bending stiffness. This is an example of geometric stiffening [34]. Ramamurti and Kielb [35] used the stress smoothing technique in which the rotational stresses were first computed and then used to update the stiffness matrix, which is then used in the eigenvalue analysis. Banerjee derived the dynamic stiffness matrix of the rotating beams including the effect of axial force for both Euler-Bernoulli and Timoshenko beam elements [36,37] and later for tapered beams [38]. According to Banerjee, the dynamic stiffness method gives exact solutions for the natural frequencies and mode shapes without making any approximation [38]. A procedure based on the method of variational iteration was presented in 2016 by Chen et al. [39] for the determination of the natural frequencies and mode shapes of rotating Euler-Bernoulli beams. They showed that the procedure is able to predict the frequencies of higher order modes with acceptable accuracy and relatively low number of iteration steps.

The above mentioned studies, concerning centrifugal stiffening of blades, neglected the flexibility of the disk. Other research works have considered centrifugal stiffening, and other rotation-induced inertia effects, in shaft-blisk assemblies. Chun and Lee developed an analytical synthesis and assumed mode method to study the effect of blisk flexibility on the dynamic behaviour of rotor systems, taking into account the Coriolis forces and centrifugal stiffening [18]. The assumed mode method, finite element method, and experimental method have been adopted by Chiu et al. in 2017 [22] in order to explore the dynamic coupling of multi flexible disk rotor system, considering centrifugal stiffening.

The finite element (FE) method is used in several studies in order to cope with the complicated configurations of rotating systems. However, shaft-blisk assemblies including Coriolis effects are rarely modelled and simulated using FE packages [26]. An investigation of different FE software packages has been done by Ruffini et al. [26] in order to examine their capabilities for predicting the dynamic behaviour of rotor systems in case of Coriolis 
and gyroscopic effects, especially with regard to the frequency splits. The FE packages used were Abaqus v6.11-13, Ansys v12.1, NX Nastran v8.5, and SAMCEF v14.1. Three test cases were presented, and the resulting Campbell diagrams from all software packages for the three cases were compared to each other and to the results from the literature. One of the test cases was the Stodola-Green rotor, which is a classic model in rotordynamics, and has strong frequency splits due to gyroscopic effects. All software predicted the frequency splits correctly, except at high speed in the vicinity of instabilities.

The non-rotational (i.e. zero-speed) mode shapes of the system obtained using FE analysis were used in a modal analysis approach by Khader and Loewy [30] to study the steady-state forced response of a flexible bladed disk supported by a shaft modelled as a massless, flexible cantilever. Although the method represented the motion using zero-speed modes, it required the derivation of the equations of motion (with rotation effects) from first principles via Lagrange's method. This in turn required the laborious derivation of kinetic energy and potential energy expressions. The part of the potential energy contributed by centrifugal stiffening was derived by discretising the centrifugal force at a number of locations along the blade and calculating its local deformation components. The zero-speed mode shapes were used again by Jacquet-Richardet et al. [15] in a modal analysis method that included gyroscopic effects, spin softening, and centrifugal stiffening. The zero-speed modal method of [15] required static analysis for the geometric stiffness matrix (that contains the centrifugal effect) which had to be repeated at each speed. This is similar to the approach used in the ANSYS rotordynamics solver [40] and can be computationally expensive (as shown in section 3 of this paper).

The above literature review highlights the need for a methodology that can efficiently include all rotation-induced inertia effects in a generic shaft-blisk system. The novel contribution of this paper is a methodology to include such effects in a generic shaft-blisk system, using 
zero-speed modal data and without the need for additional FE analysis at each speed or the derivation of equations from first principles (i.e. unlike [15] and [30] respectively).

The contribution of this paper is motivated by the need to upgrade a blisk simulator designed to generate blade tip timing (BTT) data for the development and validation of BTT algorithms [41]. In this simulator, the blisk motion is a superposition of rigid rotation, prescribed steady movement (i.e. non-oscillatory movement like axial float of the shaft), and vibratory movement due to dynamic excitation forces. This latter component of the movement is represented by a modal superposition that uses modal data from the FE model at zero rotational speed. Transformation to modal coordinates is crucial for the efficient operation of the simulator since the effective number of blisk modes required for accurate convergence of the vibration response is typically much less than the number of degrees of freedom of the FE model [41]. In the current version of the simulator (i.e. as in [41]), the modal equations of motion neglect rotational effects. This simulator was first used by the authors in [42] to investigate the effect of steady movement on BTT data. In the same year, independent researchers [43] presented a simulator that was also based on FE-derived system modes. Although the simulator in [43] accommodated gyroscopic/Coriolis effects as an optional input, these were based on a constant gyroscopic matrix (obtained from FE) applicable to a single speed only. The corresponding natural frequencies used in the modal equations included rotational effects but were only applicable for a single speed. Hence, the variation in rotational speed considered in [43] was only around $10 \%$. Using the methodology presented in this paper, the authors' blisk simulator of $[41,42]$ is upgraded to accommodate rotation-induced inertia effects, without speed restriction, by adding them to the modal equations as "external" excitations at discrete locations, so that the modal equations continue to be based on the zero-speed modes. The simulator is also upgraded to include the deflection of the shaft, but the parametric excitation effect introduced by the 
consequent in-plane vibration of the blisk centre [44] is not considered. A similar approach appears to have been taken by Ma et al. [2], Chun and Lee [18].

Section 2 starts with an overview of the existing blisk simulator (which neglects rotational effects on the dynamic response). The methodology for adding rotational effects (Coriolis/spin softening; centrifugal stiffening; gyroscopic) is then presented. Since the method requires angular displacement modal data, a method is presented to calculate such data from the translational modal data. This is a one-off computation that is needed when solid elements are used in the FE modal analysis at zero-speed (since such elements use translational degrees of freedom). The procedures for the computation of the Campbell diagram and the forced response are then described, followed by an extension of the analysis to shaft-blisk systems. Section 3 presents and discusses the results of six examples that are validated against results from the literature or from commercial software.

\section{MODELLING FOR ROTATIONAL EFFECTS}

\subsection{Overview of Existing FE-Based Modal Simulator of Blisk System [41]}

The description in this section is taken from [41], where rotation-induced inertia effects were neglected and the system consisted of a blisk only (transverse displacement of shaft neglected). If $\mathbf{u}(t)$ denotes the $3 N \times 1$ vector containing the instantaneous absolute coordinates of a subset of FE nodes $Q_{n}(n=1 \ldots N)$ covering a certain region (e.g. the tip of a given blade), the simulator computes $\mathbf{u}(t)$ as follows

$$
\mathbf{u}(t)=\mathbf{u}^{(\mathrm{rgd})}(t)+\Delta \mathbf{u}^{(\mathrm{off})}(t)+\mathrm{T}_{\mathrm{aug}}(\varphi(t)) \Delta \mathbf{u}^{(\mathrm{def})}(t)
$$

where: 
- $\mathbf{u}^{(\mathrm{rgd})}(t)$ represents the absolute blade tip positions due to rotation of the blisk as a rigid body (in the absence of deformation and any applied shifting) following a rotation $\varphi(t)$ from a reference angular position of the blisk.

- $\Delta \mathbf{u}^{\text {(off) }}(t)$ represents a prescribed steady (non-oscillatory) shift that is used to simulate “dc" bias errors on BTT data generated by the simulator [41].

- $\Delta \mathbf{u}^{(\mathrm{def})}(t)$ represents the response to the applied dynamic excitation forces.

- $\mathbf{T}_{\text {aug }}(\varphi(t))$ is a matrix that transforms displacements in a rotating coordinate frame to displacements in an inertial frame following a rigid rotation $\varphi(t)$.

Since $\Delta \mathbf{u}^{(\mathrm{def})}(t)$ is superimposed on the rigid body rotation and the prescribed steady shift, it is determined by considering the dynamic response of the non-rotating system in a fixed reference angular position. The elements of $\boldsymbol{\Delta} \mathbf{u}^{(\mathrm{def})}(t)$ are calculated using a modal transformation based on the first $H$ undamped modes of vibration of the non-rotating system in the reference angular position:

$\Delta \mathbf{u}^{(\mathrm{def})}(t)=\mathbf{H}_{\mathrm{P}} \mathbf{q}(t)$

where $\mathbf{q}(t)$ is the $H \times 1$ vector of modal co-ordinates that are governed by the modal equations of motion:

$$
\ddot{\mathbf{q}}(t)+\mathbf{C} \dot{\mathbf{q}}(t)+\mathbf{D q}(t)=\mathbf{H}_{\mathbf{f}}^{\mathrm{T}} \mathbf{f}(t)
$$

and, in Eqs. (2), (3):

- $\mathbf{H}_{\mathrm{P}}, \mathbf{H}_{\mathbf{f}}$ are modal transformation matrices whose $H$ columns are mass-normalised eigenvectors corresponding to the undamped natural circular frequencies $\varpi_{r} \quad(r=$ $1, \ldots, H)$ evaluated at the appropriate degrees of freedom.

- The matrices $\mathbf{D}$ and $\mathbf{C}$ are given by 


$$
\mathbf{D}=\operatorname{diag}\left(\left[\cdots \varpi_{r}^{2} \cdots\right]\right), \quad \mathbf{C}=\operatorname{diag}\left(\left[\cdots 2 \zeta_{r} \varpi_{r} \cdots\right]\right)
$$

where $\zeta_{r}$ is the modal damping ratio. In this work $\mathbf{C}$ is only included to suppress initial transients in the time domain integration for the forced response.

- $\mathbf{f}(t)$ is the $3 S \times 1$ vector of external dynamic excitation forces applied to the system (frozen at its reference angular position) at nodes $\mathrm{A}_{s}(s=1 \ldots S)$ in the directions of the Cartesian degrees of freedom there [45].

\subsection{Inclusion of Rotation-Induced Inertia Effects}

This section describes how the model in section 2.1 is altered to account for rotation-induced inertia effects through the addition of terms to the right hand side of Eq. (3). Fig. 1 shows the coordinate system to be used, which is compatible with the previously described system.

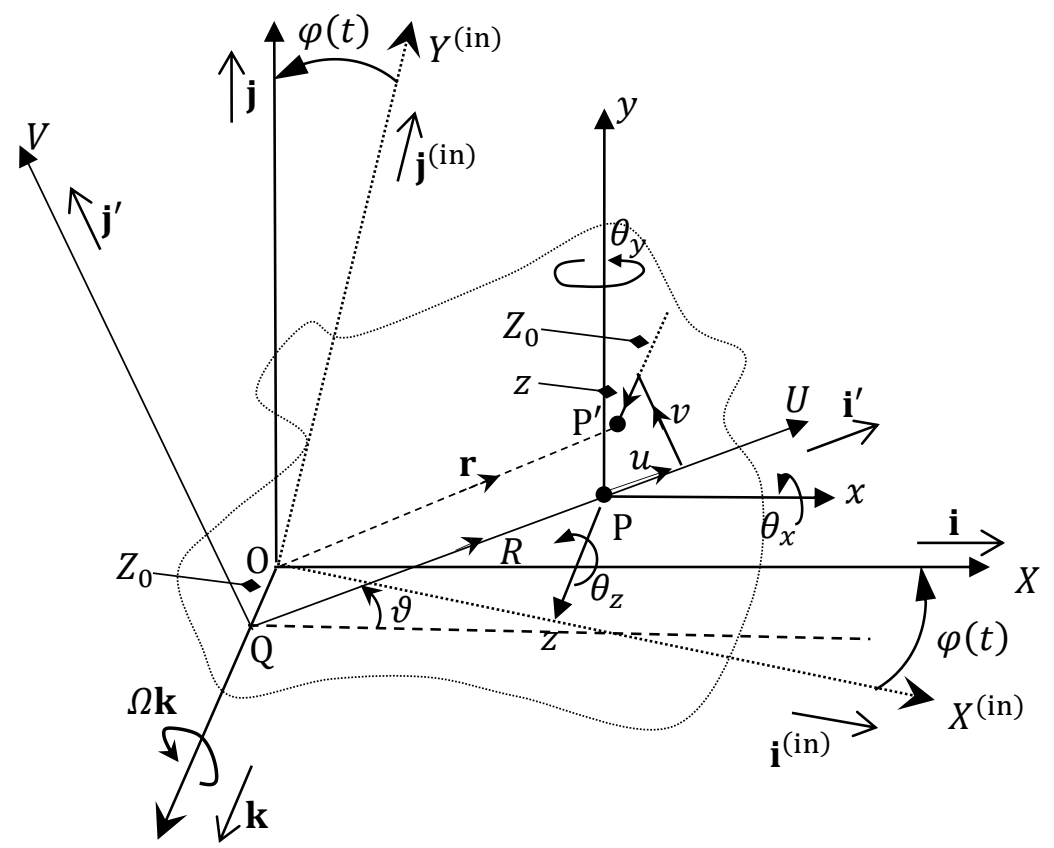

Fig. 1. Rotating structure (inertial axes $\mathrm{O} X^{(\mathrm{in})}, \mathrm{O} Y^{(\mathrm{in})}$ are horizontal, vertical respectively)

With reference to Fig. 1,

- axis $\mathrm{OZ}$ is completely fixed and along the nominal axis of rotation (independent of tilt); 
- the $X^{(\mathrm{in})}, Y^{(\mathrm{in})}$ axes through $\mathrm{O}$ are inertial, and $\mathbf{i}^{\text {(in) }}, \mathbf{j}^{\text {(in) }}$ are associated unit vectors;

- the $X, Y$ axes through $\mathrm{O}$ rotate at the spin velocity $\Omega \mathbf{k}$, where $\Omega=\dot{\varphi}$;

- $\mathbf{i}, \mathbf{j}$ are unit vectors associated with $X, Y$;

- the local Cartesian frame $(x, y, z)$ is always parallel to $X Y Z$;

- the blisk's reference angular position is $\varphi=0$.

The undeformed position $\mathrm{P}$ of an arbitrary point is fixed relative to $X Y Z$ and is located within this frame using either Cartesian coordinates $\left(X_{0}, Y_{0}, Z_{0}\right)$ or cylindrical coordinates $\left(R, \vartheta, Z_{0}\right)$. Axis $U$ coincides with the perpendicular from $\mathrm{P}$ to $\mathrm{OZ}$ and axis $V$ is normal to both $U$ and $Z$. $\mathrm{Q}$ is the point on the undeformed shaft where the perpendicular from $\mathrm{P}$ meets $\mathrm{OZ}$. It shall be assumed until section 2.5 that the transverse deflection of $Q$ (i.e. the component of its deformation in the plane of rotation $U V$ ) is zero.

The deflection of $\mathrm{P}$ to $\mathrm{P}^{\prime}$ is defined using either the local Cartesian frame $(x, y, z)$ (always parallel to $X Y Z)$ or the $U V Z$ frame $(u, v, z)$. Transformation from $u, v$ to $x, y$ and vice-versa is given by

$\left[\begin{array}{l}x \\ y\end{array}\right]=\mathbf{T}(\vartheta)\left[\begin{array}{l}u \\ v\end{array}\right],\left[\begin{array}{l}u \\ v\end{array}\right]=\mathbf{T}^{\mathrm{T}}(\vartheta)\left[\begin{array}{l}x \\ y\end{array}\right], \mathbf{T}(\vartheta)=\left[\begin{array}{cc}\cos \vartheta & -\sin \vartheta \\ \sin \vartheta & \cos \vartheta\end{array}\right]$

In Fig. 1, the angular deformations $\theta_{x}, \theta_{y}, \theta_{z}$ refer to the rotations, about the respective local Cartesian axes, of a vector $\mathbf{n}$ that is attached, and always perpendicular to, a local plane at $\mathrm{P}$, where $\mathbf{n}$ is initially parallel to axis $\mathrm{P} z$ (in case of $\theta_{x}, \theta_{y}$ ) or axis $\mathrm{P} x$ (in case of $\theta_{z}$ ).

As in Eq. (1), rigid rotation is superimposed on the dynamic deformation, and the latter is determined by modal equations with the blisk fixed in the reference angular position. Hence, the transformation to modal coordinates uses the same zero-speed mass-normalised eigenvectors used previously (which neglect rotation-induced inertia effects): 


$$
\left[\begin{array}{l}
x \\
y \\
z
\end{array}\right]=\left[\begin{array}{lll}
\psi_{x}^{(1)} & \cdots & \psi_{x}^{(H)} \\
\psi_{y}^{(1)} & \cdots & \psi_{y}^{(H)} \\
\psi_{z}^{(1)} & \cdots & \psi_{z}^{(H)}
\end{array}\right] \mathbf{q}, \quad\left[\begin{array}{l}
\theta_{x} \\
\theta_{y} \\
\theta_{z}
\end{array}\right]=\left[\begin{array}{ccc}
\psi_{\theta_{x}}^{(1)} & \cdots & \psi_{\theta_{x}}^{(H)} \\
\psi_{\theta_{y}}^{(1)} & \cdots & \psi_{\theta_{y}}^{(H)} \\
\psi_{\theta_{z}}^{(1)} & \cdots & \psi_{\theta_{z}}^{(H)}
\end{array}\right] \mathbf{q}
$$

Using this modal transformation, but accounting for rotational effects, Eq. (3) is updated as follows:

$$
\begin{aligned}
\ddot{\mathbf{q}}+\mathbf{C} \dot{\mathbf{q}}+\mathbf{D q}= & \mathbf{H}_{\mathbf{f}}^{\mathrm{T}} \mathbf{f}(t) \\
& +\sum_{\mathrm{P}_{k}}\left\{\mathbf{H}_{\mathbf{h}_{k}}^{\mathrm{T}}\left[\mathbf{h}_{k}(\mathbf{q}, \dot{\mathbf{q}}, \Omega, \dot{\Omega})+\mathbf{h}_{\mathbf{0}_{k}}(\Omega, \dot{\Omega})\right]+\mathbf{H}_{\mathbf{m}_{k}}^{\mathrm{T}} \mathbf{m}_{k}(\mathbf{q}, \Omega)+\mathbf{H}_{\mathbf{g}_{k}}^{\mathrm{T}} \mathbf{g}_{k}(\dot{\mathbf{q}}, \Omega)\right\}
\end{aligned}
$$

In the above approach, the blisk is divided into radial/angular segments (Fig. 2) and the rotational inertia effects from the individual segments, with mass centres at selected FE nodes $\mathrm{P}_{k}(k=1 \ldots K)$, are summed over the entire system and added as additional "external" forces to the modal equation. The nodes $\mathrm{P}_{k}$ are selected from the nodes of the FE mesh used in the non-rotational free undamped vibration analysis (for the mode shapes) so as to coincide as closely as possible with the mass centres of the radial/angular segments in Fig. 2. In Eq. (8):

- $\mathbf{h}_{k}$ is a $2 \times 1$ force vector that contains the Coriolis and spin-softening effects;

- $\mathbf{m}_{k}$ is a $3 \times 1$ moment vector that contains the centrifugal stiffening effect;

- $\mathbf{g}_{k}$ is a $2 \times 1$ moment vector that contains the gyroscopic effect;

- $\mathbf{h}_{\mathbf{0}_{k}}$ is a $2 \times 1$ force vector that is not vibration-dependent, unlike the above vectors.

- $\quad \mathbf{H}_{\mathbf{h}_{k}}, \mathbf{H}_{\mathbf{m}_{k}}, \mathbf{H}_{\mathbf{g}_{k}}$ are (speed-independent) modal matrices whose $H$ columns are the massnormalised eigenvectors evaluated at the degrees of freedom associated with $\mathbf{h}_{k}, \mathbf{m}_{k}$, and $\mathbf{g}_{k}$ respectively.

Expressions for the vectors $\mathbf{h}_{k}, \mathbf{h}_{\mathbf{0}_{k}}, \mathbf{m}_{k}, \mathbf{g}_{k}$ are derived in the following subsections. 


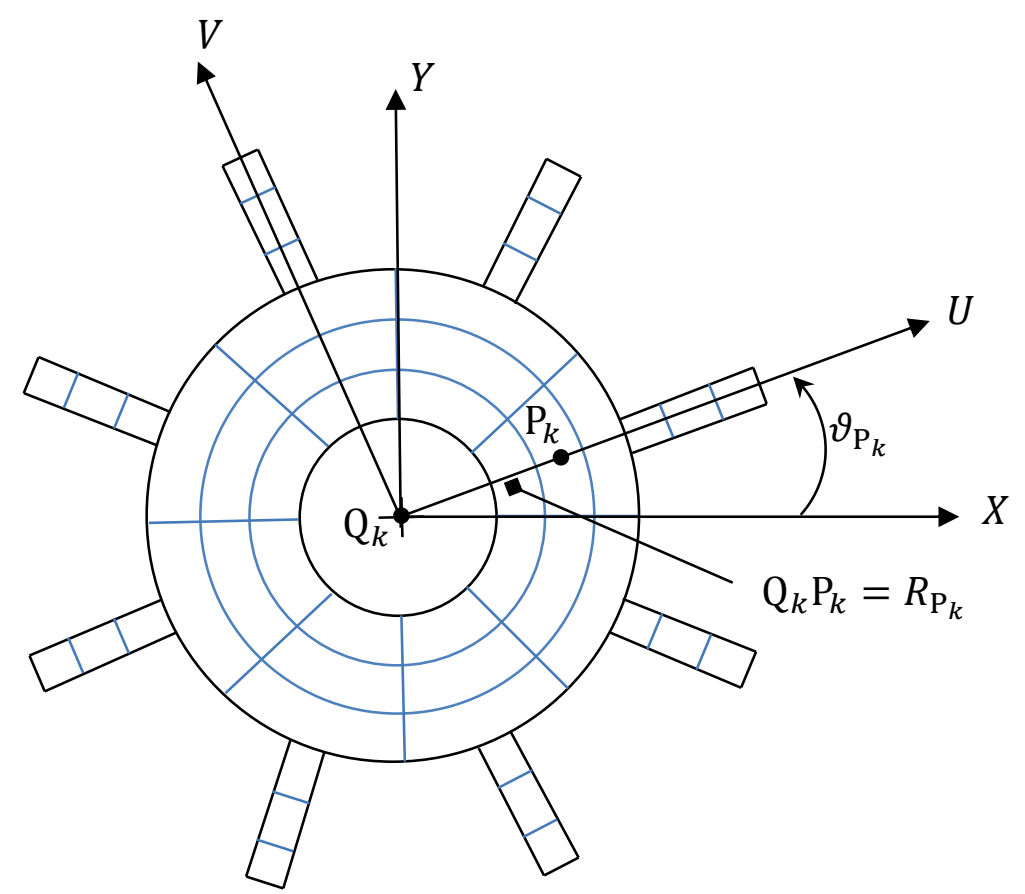

Fig. 2. Blisk radial/angular divisions for rotational inertia effects, where a generic segment has mass centre $\mathrm{P}_{k}$ and mass $\Delta m_{\mathrm{P}_{k}}$ (same nomenclature as Figure 1 but with $\mathrm{P}$ replaced by $\mathrm{P}_{k}$ and $\mathrm{Q}$ replaced by $\mathrm{Q}_{k}$, the shadow point of $\mathrm{P}_{k}$ on axis of rotation).

\subsubsection{Coriolis and Spin Softening Effects}

With reference to Fig. 1, the position vector of an arbitrary point $\mathrm{P}^{\prime}$ that was at $\mathrm{P}$ prior to deformation is given by

$$
\mathbf{r}(t)=(R+u(t)) \mathbf{i}^{\prime}+v(t) \mathbf{j}^{\prime}+\left(Z_{0}+z(t)\right) \mathbf{k}
$$

where the unit vectors $\mathbf{i}^{\prime}, \mathbf{j}^{\prime}$ vary with time:

$\frac{\mathrm{d}}{\mathrm{d} t}\left(\mathbf{i}^{\prime}\right)=\Omega \mathbf{k} \times \mathbf{i}^{\prime}=\Omega \mathbf{j}^{\prime} \quad, \quad \frac{\mathrm{d}}{\mathrm{d} t}\left(\mathbf{j}^{\prime}\right)=\Omega \mathbf{k} \times \mathbf{j}^{\prime}=-\Omega \mathbf{i}^{\prime}$

Differentiating Eq. (9) twice with respect to time, and noting Eqs. (10a,b), results in:

$$
\begin{aligned}
& \ddot{\mathbf{r}}=\ddot{u} \mathbf{i}^{\prime}+\ddot{v} \mathbf{j}^{\prime}+\ddot{z} \mathbf{k}-\left[(R+u(t)) \Omega^{2}+2 \dot{v} \Omega+v(t) \dot{\Omega}\right] \mathbf{i}^{\prime}+[(R+u(t)) \dot{\Omega}+2 \dot{u} \Omega- \\
& \left.v(t) \Omega^{2}\right] \mathbf{j}^{\prime}
\end{aligned}
$$


It is evident that the term $\mathbf{h}_{k}+\mathbf{h}_{\mathbf{0}_{k}}$ in Eq. (8) is the inertial reaction (in Cartesian components) to the rotational acceleration terms in Eq. (11). Hence, applying the transformation Eq. (6a) to the last two terms of Eq. (11), and denoting the mass of the radial/angular segment of $\mathrm{P}_{k}$ as $\Delta m_{\mathrm{P}_{k}}$, and relating Fig. 2 to Fig. 1, one obtains

$\mathbf{h}_{k}+\mathbf{h}_{\mathbf{0}_{k}}=\Delta m_{\mathrm{P}_{k}} \mathbf{T}\left(\vartheta_{\mathrm{P}_{k}}\right)\left[\begin{array}{c}\left(R_{\mathrm{P}_{k}}+u_{\mathrm{P}_{k}}\right) \Omega^{2}+2 \dot{v}_{\mathrm{P}_{k}} \Omega+v_{\mathrm{P}_{k}} \dot{\Omega} \\ -\left(R_{\mathrm{P}_{k}}+u_{\mathrm{P}_{k}}\right) \dot{\Omega}-2 \dot{u}_{\mathrm{P}_{k}} \Omega+v_{\mathrm{P}_{k}} \Omega^{2}\end{array}\right]$

from which

$$
\mathbf{h}_{\mathbf{0}_{k}}=\Delta m_{\mathrm{P}_{k}} \mathbf{T}\left(\vartheta_{\mathrm{P}_{k}}\right)\left[\begin{array}{l}
R_{\mathrm{P}_{k}} \Omega^{2} \\
-R_{\mathrm{P}_{k}} \dot{\Omega}
\end{array}\right]
$$

Expressing the $u, v$ terms in Eq. (12) in terms of $x, y$ (using Eq. (6b)), and then the latter to modal coordinates, yields the following expression for $\mathbf{h}_{k}$ :

$$
\mathbf{h}_{k}=\Delta m_{\mathrm{P}_{k}} \mathbf{T}\left(\vartheta_{\mathrm{P}_{k}}\right)\left\{\left[\begin{array}{cc}
0 & 2 \Omega \\
-2 \Omega & 0
\end{array}\right] \mathbf{T}^{\mathrm{T}}\left(\vartheta_{\mathrm{P}_{k}}\right) \mathbf{H}_{\mathbf{h}_{k}} \dot{\mathbf{q}}+\left[\begin{array}{cc}
\Omega^{2} & \dot{\Omega} \\
-\dot{\Omega} & \Omega^{2}
\end{array}\right] \mathbf{T}^{\mathrm{T}}\left(\vartheta_{\mathrm{P}_{k}}\right) \mathbf{H}_{\mathbf{h}_{k}} \mathbf{q}\right\}
$$

Following Eq. (7a), the matrix $\mathbf{H}_{\mathbf{h}_{k}}$ (used in Eq. (14) and Eq. (8)) is given by:

$$
\mathbf{H}_{\mathbf{h}_{k}}=\left[\begin{array}{lll}
\psi_{x_{\mathrm{P}_{k}}}^{(1)} & \cdots & \psi_{x_{\mathrm{P}_{k}}}^{(H)} \\
\psi_{y_{\mathrm{P}_{k}}}^{(1)} & \cdots & \psi_{y_{\mathrm{P}_{k}}}^{(H)} \\
\psi_{\mathrm{P}_{\mathrm{P}_{k}}}^{(1)} & \cdots & \psi_{z_{\mathrm{P}_{k}}}^{(H)}
\end{array}\right]
$$




\subsubsection{Centrifugal Stiffening}

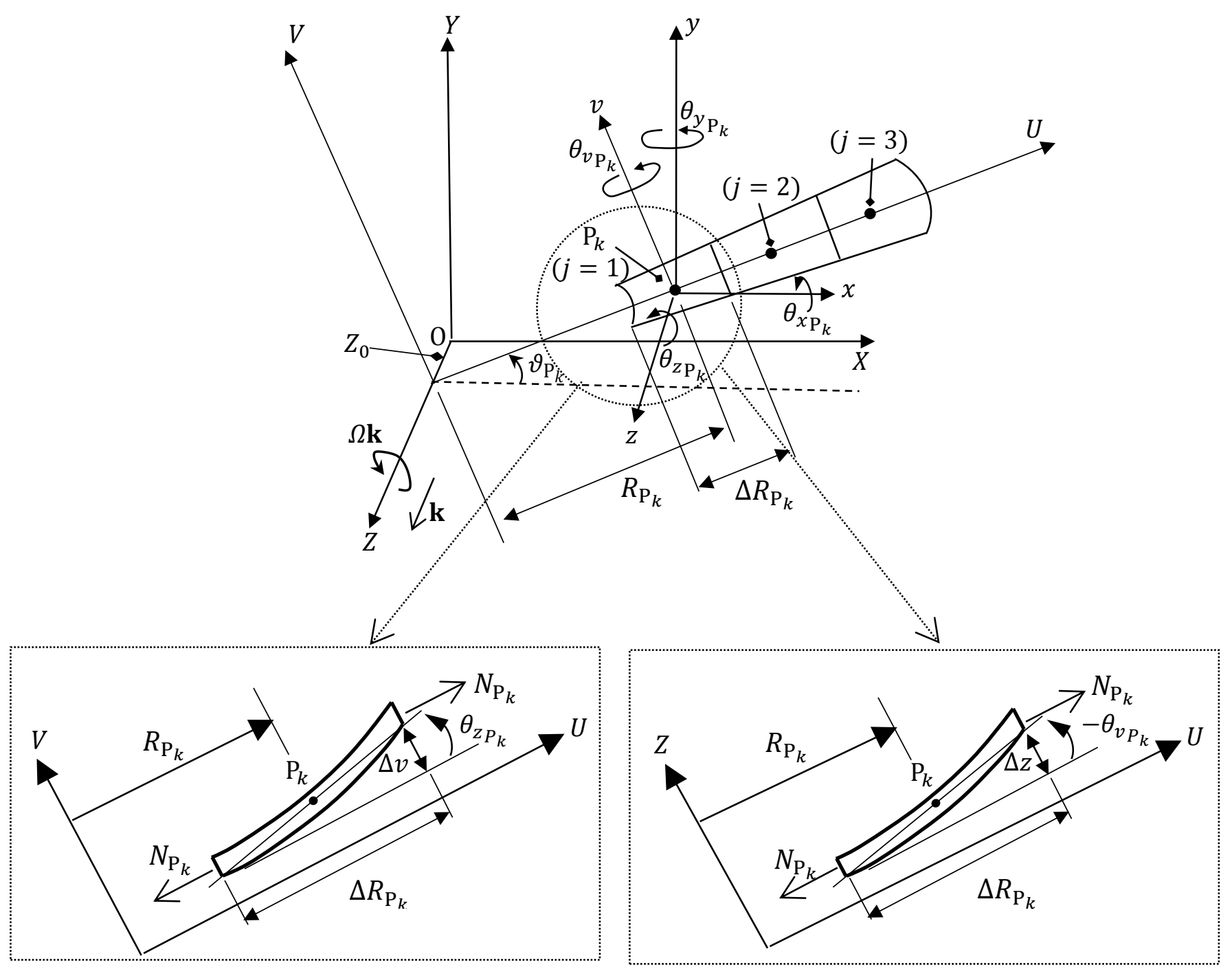

Fig. 3. Modelling of centrifugal stiffening effect

The centrifugal tension $N_{\mathrm{P}_{k}}$ on a radial/angular segment with mass centre $\mathrm{P}_{k}\left(R_{\mathrm{P}_{k}}, \vartheta_{\mathrm{P}_{k}}, Z_{0_{\mathrm{P}_{k}}}\right)$ of radial extent $\Delta R_{\mathrm{P}_{k}}$ (see Fig. 3) is given by: 


$$
N_{\mathrm{P}_{k}}=\left.\Omega^{2}\left\{\sum_{j=1}^{J} \Delta m_{j} R_{j}\right\}\right|_{R_{1}=R_{P_{k}}}
$$

where the summation is applied to radial/angular segments of respective masses $\Delta m_{j}$ and radial positions $R_{j}$, with mass centres at nodes located along the same angular and axial position of $\mathrm{P}_{k}\left(\vartheta_{\mathrm{P}_{k}}, Z_{\mathrm{P}_{\mathrm{P}}}\right)$, starting from $\mathrm{P}_{k}$ (i.e. $\left.R_{1}=R_{\mathrm{P}_{k}}\right)$ and proceeding radially outward. With reference to Fig. 3, let the deflections $v, z$ increase by $\Delta v, \Delta z$ respectively over the radial extent $\Delta R_{\mathrm{P}_{k}}$. The centrifugal tension $N_{\mathrm{P}_{k}}$ stiffens the segment by inducing moments in the edge-wise $(U V)$ and flap-wise $(U Z)$ planes $\left(M_{z_{\mathrm{P}_{k}}}, M_{v_{\mathrm{P}_{k}}}\right.$ respectively) which oppose the corresponding angular deformations $\theta_{\mathrm{Z}_{\mathrm{P}}}, \theta_{v_{\mathrm{P}_{k}}}$ :

$$
\begin{gathered}
M_{z_{\mathrm{P}_{k}}}=-N_{\mathrm{P}_{k}} \Delta v=-N_{\mathrm{P}_{k}} \Delta R_{\mathrm{P}_{k}} \frac{\Delta v}{\Delta R_{\mathrm{P}_{k}}}=-N_{\mathrm{P}_{k}} \Delta R_{\mathrm{P}_{k}} \theta_{\mathrm{P}_{\mathrm{P}_{k}}} \\
M_{v_{\mathrm{P}_{k}}}=N_{\mathrm{P}_{k}} \Delta z=N_{\mathrm{P}_{k}} \Delta R_{\mathrm{P}_{k}} \frac{\Delta z}{\Delta R_{\mathrm{P}_{k}}}=-N_{\mathrm{P}_{k}} \Delta R_{\mathrm{P}_{k}} \theta_{\mathrm{P}_{\mathrm{P}_{k}}}
\end{gathered}
$$

Considering Eq. (18) and applying the transformation of Eq. (6a) and then Eq. (6b):

$$
\begin{aligned}
{\left[\begin{array}{l}
M_{x_{\mathrm{P}_{k}}} \\
M_{y_{\mathrm{P}_{k}}}
\end{array}\right]=M_{v_{\mathrm{P}_{k}}}\left[\begin{array}{c}
-\sin \vartheta_{\mathrm{P}_{k}} \\
\cos \vartheta_{\mathrm{P}_{k}}
\end{array}\right]=-N_{\mathrm{P}_{k}} \Delta R_{\mathrm{P}_{k}} \theta_{\mathrm{P}_{\mathrm{P}_{k}}}\left[\begin{array}{c}
-\sin \vartheta_{\mathrm{P}_{k}} \\
\cos \vartheta_{\mathrm{P}_{k}}
\end{array}\right] } \\
=-N_{\mathrm{P}_{k}} \Delta R_{\mathrm{P}_{k}}\left(-\theta_{x_{\mathrm{P}_{k}}} \sin \vartheta_{\mathrm{P}_{k}}+\theta_{y_{\mathrm{P}_{k}}} \cos \vartheta_{\mathrm{P}_{k}}\right)\left[\begin{array}{c}
-\sin \vartheta_{\mathrm{P}_{k}} \\
\cos \vartheta_{\mathrm{P}_{k}}
\end{array}\right]
\end{aligned}
$$

Hence, combining Eqs. (19) and (17), the centrifugal stiffening moment vector in Eq. (8) is given by:

$$
\mathbf{m}_{k}=-N_{\mathrm{P}_{k}} \Delta R_{\mathrm{P}_{k}} \widetilde{\mathbf{T}}\left(\vartheta_{\mathrm{P}_{k}}\right)\left[\begin{array}{lll}
\theta_{\mathrm{P}_{\mathrm{P}_{k}}} & \theta_{y_{\mathrm{P}_{k}}} & \theta_{\mathrm{Z}_{\mathrm{P}}}
\end{array}\right]^{\mathrm{T}}=-N_{\mathrm{P}_{k}} \Delta R_{\mathrm{P}_{k}} \breve{\mathbf{T}}\left(\vartheta_{\mathrm{P}_{k}}\right) \mathbf{H}_{\mathbf{m}_{k}} \mathbf{q}
$$


Where

$$
\widetilde{\mathbf{T}}\left(\vartheta_{\mathrm{P}_{k}}\right)=\left[\begin{array}{ccc}
\sin ^{2} \vartheta_{\mathrm{P}_{k}} & -\sin \vartheta_{\mathrm{P}_{k}} \cos \vartheta_{\mathrm{P}_{k}} & 0 \\
-\sin \vartheta_{\mathrm{P}_{k}} \cos \vartheta_{\mathrm{P}_{k}} & \cos ^{2} \vartheta_{\mathrm{P}_{k}} & 0 \\
0 & 0 & 1
\end{array}\right]
$$

and, from Eq. (7b):

$$
\mathbf{H}_{\mathbf{m}_{k}}=\left[\begin{array}{lll}
\psi_{\theta_{x_{\mathrm{P}_{k}}}}^{(1)} & \cdots & \psi_{\theta_{x_{\mathrm{P}_{k}}}}^{(H)} \\
\psi_{\theta_{y_{\mathrm{P}_{k}}}}^{(1)} & \cdots & \psi_{\theta_{\mathrm{P}_{\mathrm{P}}}^{(H)}}^{(1)} \\
\psi_{\theta_{\mathrm{z}_{k}}}^{(1)} & \cdots & \psi_{\theta_{\mathrm{z}_{\mathrm{P}}}}^{(H)}
\end{array}\right]
$$

\subsubsection{Gyroscopic Effects}

The angular momentum of a radial/angular segment with mass centre $\mathrm{P}_{k}$ is given by

$$
\mathbf{L}_{k}=\Delta I_{\mathrm{P}_{k}} \Omega \mathbf{n}_{k}
$$

where $\Delta I_{\mathrm{P}_{k}}$ is the moment of inertia of the segment about the nominal axis of rotation $\mathrm{OZ}$ (Fig. 1) and $\mathbf{n}_{k}$ is a unit vector that is attached, and always perpendicular to, a local plane at $\mathrm{P}_{k}$, where $\mathbf{n}_{k}$ is originally parallel to $\mathrm{OZ}$. Due to deformation, $\mathbf{n}_{k}$ is given by

$$
\mathbf{n}_{k}=\mathbf{k}+\mathbf{n}_{\mathrm{def}_{k}}(t)
$$

where $\mathbf{n}_{\text {def }}(t)$ is the variable part of $\mathbf{n}_{k}$ due to its change in direction at a precession velocity of $\dot{\theta}_{x_{P_{k}}} \mathbf{i}+\dot{\theta}_{y_{P_{k}}} \mathbf{j}$.

Hence 
$\dot{\mathbf{L}}_{k}=\Delta I_{\mathrm{P}_{k}} \Omega \dot{\mathbf{n}}_{k}=\Delta I_{\mathrm{P}_{k}} \Omega\left(\dot{\theta}_{x_{P_{k}}} \mathbf{i}+\dot{\theta}_{y_{P_{k}}} \mathbf{j}\right) \times \mathbf{n}_{k}$

Neglecting second order quantities from Eq. (25) by assuming $\mathbf{i} \times \mathbf{n}_{k} \approx-\mathbf{j}$ and $\mathbf{j} \times \mathbf{n}_{k} \approx \mathbf{i}$, one obtains the gyroscopic moment reaction vector $\mathbf{g}_{k}$ in Eq. (8) as:

$\mathbf{g}_{k}=-\dot{\mathbf{L}}_{k}=\Omega\left[\begin{array}{cc}0 & -\Delta I_{\mathrm{P}_{k}} \\ \Delta I_{\mathrm{P}_{k}} & 0\end{array}\right]\left[\begin{array}{l}\dot{\theta}_{x_{\mathrm{P}_{k}}} \\ \dot{\theta}_{y_{\mathrm{P}_{k}}}\end{array}\right]=\Omega\left[\begin{array}{cc}0 & -\Delta I_{\mathrm{P}_{k}} \\ \Delta I_{\mathrm{P}_{k}} & 0\end{array}\right] \mathbf{H}_{\mathbf{g}_{k}} \dot{\mathbf{q}}$

where, from Eq. (7b):

$\mathbf{H}_{\mathbf{g}_{k}}=\left[\begin{array}{lll}\psi_{\theta_{x_{\mathrm{P}_{k}}}}^{(1)} & \cdots & \psi_{\theta_{x_{\mathrm{P}_{k}}}}^{(H)} \\ \psi_{\theta_{y_{\mathrm{P}_{k}}}}^{(1)} & \cdots & \psi_{\theta_{y_{\mathrm{P}_{k}}}}^{(H)}\end{array}\right]$

Notice that, for the simple case where the blisk is rigid and deformation only occurs in the shaft and/or bearings, the time-varying vectors $\mathbf{n}_{k}$ across the blisk would all be in the same direction at any given instant, which means that the matrices $\mathbf{H}_{\mathbf{g}_{k}}$ would be identical for all points $\mathrm{P}_{k}$ on the (rigid) blisk.

\subsection{Calculation of modal angular displacements}

The eigenvectors computed from FE analysis using solid elements express the mode shapes in terms of the Cartesian translational deflections at the nodes i.e. $(x, y, z)$ and do not give angular deformation data i.e. $\left(\theta_{x}, \theta_{y}, \theta_{z}\right)$. This section describes how the translational deflections of the mode shape are used to obtain the corresponding angular deformation data required for the matrices of Eqs. (22) and (27). 


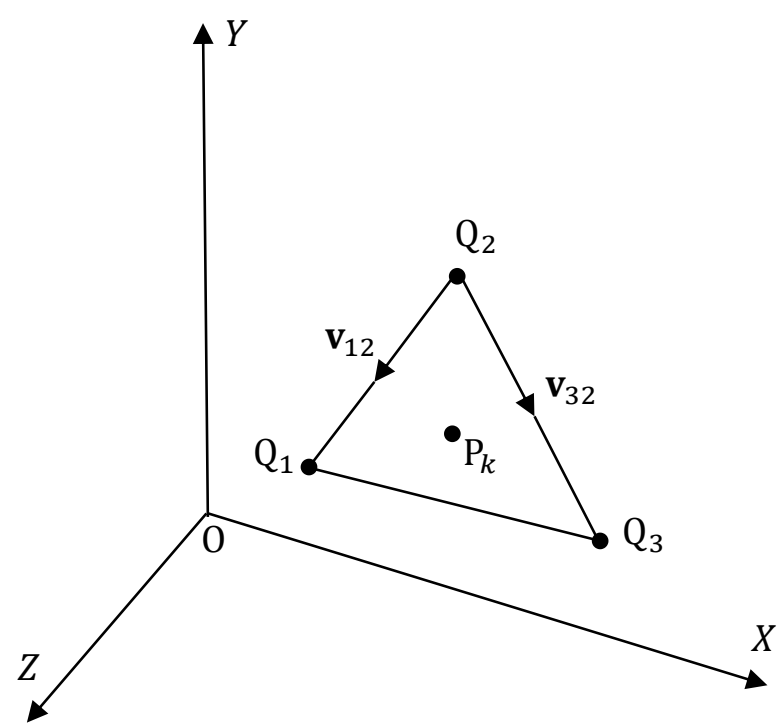

Fig. 4. Local plane through segment mass centre $\mathrm{P}_{k}$ formed by three adjacent nodes

For each FE node corresponding to the centre of mass $\left(\mathrm{P}_{k}\right)$ of one of the blisk inertia sectors, three different FE nodes $\mathrm{Q}_{1}, \mathrm{Q}_{2}, \mathrm{Q}_{3}$ with respective coordinates $\left(X_{0_{\mathrm{Q}_{i}}} Y_{0_{\mathrm{Q}_{i}}}{ }{ }_{{ }_{0} \mathrm{Q}_{i}}\right), i=1,2,3$, are selected around $\mathrm{P}_{k}$ such that they form a plane parallel to $X Y$ plane and containing $\mathrm{P}_{k}$ as shown in Fig. 4. The normal vector $\mathbf{n}$ of the plane is therefore parallel to the $Z$-axis before deformation. The $\theta_{x}, \theta_{y}$ deformations at $\mathrm{P}_{k}$ corresponding to known translational deformations at $\mathrm{Q}_{i}\left(x_{\mathrm{Q}_{i}}, y_{\mathrm{Q}_{i}}, z_{\mathrm{Q}_{i}}\right)$ can be determined from the rotation of $\mathbf{n}$ about the $y$ and $x$ axes:

$$
\theta_{x_{\mathrm{P}_{k}}}=-\frac{n_{y}}{n_{z}} \quad, \quad \theta_{y_{\mathrm{P}_{k}}}=\frac{n_{x}}{n_{z}}
$$

where

$$
\begin{gathered}
\mathbf{n}=n_{x} \mathbf{i}+n_{x} \mathbf{j}+n_{x} \mathbf{k}=\mathbf{v}_{12} \times \mathbf{v}_{32} \\
\mathbf{v}_{12}=\left(X_{\mathrm{Q}_{1}}-X_{\mathrm{Q}_{2}}\right) \mathbf{i}+\left(Y_{\mathrm{Q}_{1}}-Y_{\mathrm{Q}_{2}}\right) \mathbf{j}+\left(Z_{\mathrm{Q}_{1}}-Z_{\mathrm{Q}_{2}}\right) \mathbf{k} \\
\mathbf{v}_{32}=\left(X_{\mathrm{Q}_{3}}-X_{\mathrm{Q}_{2}}\right) \mathbf{i}+\left(Y_{\mathrm{Q}_{3}}-Y_{\mathrm{Q}_{2}}\right) \mathbf{j}+\left(Z_{\mathrm{Q}_{3}}-Z_{\mathrm{Q}_{2}}\right) \mathbf{k}
\end{gathered}
$$




$$
X_{\mathrm{Q}_{i}}=X_{0_{\mathrm{Q}_{i}}}+x_{\mathrm{Q}_{i}}, Y_{\mathrm{Q}_{i}}=Y_{0_{\mathrm{Q}_{i}}}+y_{\mathrm{Q}_{i}}, Z_{\mathrm{Q}_{i}}=Z_{0 \mathrm{Q}_{i}}+z_{\mathrm{Q}_{i}}
$$

The translational and angular deformations at nodes $\mathrm{P}_{k}$ and $\mathrm{Q}_{i}$ in response to a harmonic force $F_{\mathrm{ZP}_{k}}(t)=\operatorname{Re}\left\{\widetilde{F}_{\mathrm{P}_{k}} \mathrm{e}^{\mathrm{j} \omega t}\right\}$ applied at point $\mathrm{P}_{k}$ in the $z$-direction are given by the following receptance frequency response functions (FRFs):

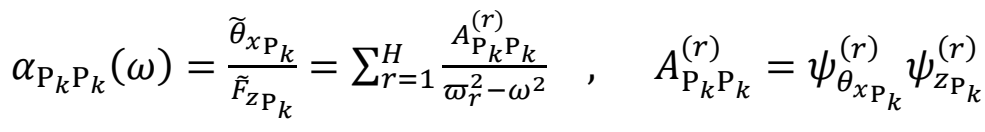

$$
\begin{aligned}
& \beta_{\mathrm{P}_{k} \mathrm{P}_{k}}(\omega)=\frac{\widetilde{\theta}_{\mathrm{P}_{k}}}{\tilde{F}_{\mathrm{P}_{k}}}=\sum_{r=1}^{H} \frac{B_{\mathrm{P}_{k} \mathrm{P}_{k}}^{(r)}}{\omega_{r}^{2}-\omega^{2}} \quad, \quad B_{\mathrm{P}_{k} \mathrm{P}_{k}}^{(r)}=\psi_{\theta_{y_{\mathrm{P}_{k}}}}^{(r)} \psi_{\mathrm{P}_{\mathrm{P}_{k}}}^{(r)} \\
& \gamma_{\mathrm{Q}_{i} \mathrm{P}_{k}}(\omega)=\frac{{\tilde{Q_{Q}}}_{\mathrm{Q}_{i}}}{\tilde{F}_{\mathrm{z}_{k}}}=\sum_{r=1}^{H} \frac{C_{\mathrm{Q}_{i} \mathrm{P}_{k}}^{(r)}}{\varpi_{r}^{2}-\omega^{2}} \quad, \quad C_{\mathrm{Q}_{i} \mathrm{P}_{k}}^{(r)}=\psi_{\chi_{\mathrm{Q}_{i}}}^{(r)} \psi_{\mathrm{z}_{\mathrm{P}_{k}}}^{(r)} \\
& \sigma_{\mathrm{Q}_{i} \mathrm{P}_{k}}(\omega)=\frac{\tilde{y}_{\mathrm{Q}_{i}}}{\tilde{F}_{\mathrm{P}_{\mathrm{P}}}}=\sum_{r=1}^{H} \frac{D_{\mathrm{Q}_{\mathrm{P}}}^{(r)}}{\bar{\omega}_{r}^{2}-\omega^{2}} \quad, \quad D_{\mathrm{Q}_{i} \mathrm{P}_{k}}^{(r)}=\psi_{{\mathrm{Q}_{i}}_{\mathrm{Q}}}^{(r)} \psi_{\mathrm{P}_{\mathrm{P}_{k}}}^{(r)} \\
& \varepsilon_{\mathrm{Q}_{i} \mathrm{P}_{k}}(\omega)=\frac{\tilde{Q}_{\mathrm{Q}_{i}}}{\tilde{F}_{\mathrm{P}_{k}}}=\sum_{r=1}^{H} \frac{E_{\mathrm{Q}_{i} \mathrm{P}_{k}}^{(r)}}{\varpi_{r}^{2}-\omega^{2}} \quad, \quad E_{\mathrm{Q}_{i} \mathrm{P}_{k}}^{(r)}=\psi_{z_{\mathrm{Q}_{i}}}^{(r)} \psi_{\mathrm{P}_{\mathrm{P}_{k}}}^{(r)}
\end{aligned}
$$

where:

- $\quad x_{\mathrm{P}_{k}}(t)=\operatorname{Re}\left\{\tilde{x}_{\mathrm{P}_{k}} \mathrm{e}^{\mathrm{j} \omega t}\right\}, \ldots$ etc, $\theta_{x_{\mathrm{P}_{k}}}(t)=\operatorname{Re}\left\{\tilde{\theta}_{x_{\mathrm{P}_{k}}} \mathrm{e}^{\mathrm{j} \omega t}\right\}, \ldots$ etc;

- $x_{\mathrm{Q}_{i}}(t)=\operatorname{Re}\left\{\tilde{x}_{\mathrm{Q}_{i}} \mathrm{e}^{\mathrm{j} \omega t}\right\}, \ldots$. etc, $(i=1,2.3)$;

- $\varpi_{r}, r=1 \ldots H$, are the undamped natural circular frequencies.

The only unknowns in Eqs. (30-34) are the modal angular deformations $\psi_{\theta_{x_{\mathrm{P}_{k}}}}^{(r)}, \psi_{\theta_{y_{\mathrm{P}_{k}}}}^{(r)}$, all other modal displacements being translational and output from FE analysis. The values of $\psi_{\theta_{x_{\mathrm{P}_{k}}}}^{(r)}, \psi_{\theta_{{y_{\mathrm{P}}}_{k}}^{(r)}}^{(r e}$ are determined as follows. 
1. Set $\widetilde{F}_{\mathrm{Z}_{\mathrm{P}}}=1$.

2. For a given excitation frequency $\omega$, calculate the amplitudes $\tilde{x}_{\mathrm{Q}_{i}}, \tilde{y}_{\mathrm{Q}_{i}}, \tilde{z}_{\mathrm{Q}_{i}}(i=1,2.3)$ from Eqs. (32-34).

3. Determine the corresponding amplitudes $\tilde{\theta}_{x_{\mathrm{P}_{k}}}, \tilde{\theta}_{y_{\mathrm{P}_{k}}}$ (and hence the FRFs $\alpha_{\mathrm{P}_{k} \mathrm{P}_{k}}(\omega)$, $\beta_{\mathrm{P}_{k} \mathrm{P}_{k}}(\omega)$ on the left hand sides of Eqs. (30a), (31a)) using Eqs. (28) and (29) with $x_{\mathrm{Q}_{i}}, y_{\mathrm{Q}_{i}}, z_{\mathrm{Q}_{i}}$ replaced by $\tilde{x}_{\mathrm{Q}_{i}}, \tilde{y}_{\mathrm{Q}_{i}}, \tilde{z}_{\mathrm{Q}_{i}}$.

4. Repeat steps 1-3 for excitation frequencies $\omega=\omega_{n}, n=1 \ldots N \quad(N>H)$ thus determining the FRF values $\alpha_{\mathrm{P}_{k} \mathrm{P}_{k}}\left(\omega_{n}\right), \beta_{\mathrm{P}_{k} \mathrm{P}_{k}}\left(\omega_{n}\right)$ on the left hand sides of Eqs. (30a), (31a).

5. Determine $A_{\mathrm{P}_{k} \mathrm{P}_{k}}^{(r)}$ on the right hand side of Eq. (30a) by solving the least-squares problem:

$$
\left[\begin{array}{c}
\alpha_{\mathrm{P}_{k} \mathrm{P}_{k}}\left(\omega_{1}\right) \\
\alpha_{\mathrm{P}_{k} \mathrm{P}_{k}}\left(\omega_{2}\right) \\
\vdots \\
\alpha_{\mathrm{P}_{k} \mathrm{P}_{k}}\left(\omega_{N}\right)
\end{array}\right]_{N \times 1}=\left[\begin{array}{cccc}
\frac{1}{\varpi_{1}^{2}-\omega_{1}^{2}} & \frac{1}{\varpi_{2}^{2}-\omega_{1}^{2}} & \cdots & \frac{1}{\varpi_{H}^{2}-\omega_{1}^{2}} \\
\frac{1}{\varpi_{1}^{2}-\omega_{2}^{2}} & \frac{1}{\varpi_{2}^{2}-\omega_{2}^{2}} & \cdots & \frac{1}{\varpi_{H}^{2}-\omega_{2}^{2}} \\
\vdots & \vdots & \cdots & \vdots \\
\frac{1}{\varpi_{1}^{2}-\omega_{N}^{2}} & \frac{1}{\varpi_{2}^{2}-\omega_{N}^{2}} & \cdots & \frac{1}{\varpi_{H}^{2}-\omega_{N}^{2}}
\end{array}\right]_{N \times H}\left[\begin{array}{c}
A_{\mathrm{P}_{k} \mathrm{P}_{k}}^{(1)} \\
A_{\mathrm{P}_{k} \mathrm{P}_{k}}^{(2)} \\
\vdots \\
A_{\mathrm{P}_{k} \mathrm{P}_{k}}^{(H)}
\end{array}\right]_{H \times 1}
$$

6. From Eq. (30b), determine $\psi_{\theta_{x_{\mathrm{P}_{k}}}^{(r)}}^{\text {. }}$

7. Determine $\psi_{\theta_{y_{\mathrm{P}_{k}}}}^{(r)}$ by repeating steps 5-6 for $B_{\mathrm{P}_{k} \mathrm{P}_{k}}^{(r)}$ using Eqs. (31a) and (31b).

A similar procedure is followed to obtain the modal angular deformations $\psi_{\theta_{\mathrm{zP}_{k}}}^{(r)}$, with the following modifications:

- The nodes $\mathrm{Q}_{1}, \mathrm{Q}_{2}, \mathrm{Q}_{3}$ are chosen to lie in a plane which is parallel to the $Y Z$ plane. 
- Equations (30), (31) are replaced by the FRF relating ${\widetilde{F_{x}}}_{\mathrm{P}_{k}}$ with $\theta_{z_{\mathrm{P}_{k}}}$, and Eqs. (32-34) modified by replacing subscript " $\mathrm{Z}_{\mathrm{P}_{k}}$ " with " $\mathrm{P}_{\mathrm{P}_{k}}$ ".

- Replace Eq. (28) with the following formula:

$$
\theta_{\mathrm{P}_{k}}=\frac{n_{y}}{n_{x}}
$$

\subsection{Campbell Diagrams and Forced Response}

Substituting, Eqs. (13), (14), (20), and (26) into Eq. (8), results in the following modal equation:

$\ddot{\mathbf{q}}+\left\{\mathbf{C}+\Omega\left(\mathbf{G}+\mathbf{A}_{1}\right)\right\} \dot{\mathbf{q}}+\left\{\mathbf{D}+\Omega^{2}\left(\mathbf{N}+\mathbf{A}_{2}\right)+\dot{\Omega} \mathbf{A}_{3}\right\} \mathbf{q}=\mathbf{H}_{\mathbf{f}}^{\mathrm{T}} \mathbf{f}(t)+\mathbf{a}_{0}\left(\Omega^{2}, \dot{\Omega}\right)$

where the speed-independent matrices $\mathbf{G}, \mathbf{A}_{1}, \mathbf{N}, \mathbf{A}_{2}, \mathbf{A}_{3}$ respectively contain the gyroscopic, Coriolis, centrifugal stiffening, spin-softening and angular acceleration effects. These matrices, along with the speed-dependent vector $\mathbf{a}_{0}$ are given by the following expressions.

$$
\begin{gathered}
\mathbf{G}=\sum_{\mathrm{P}_{k}}\left\{\Delta I_{\mathrm{P}_{k}} \mathbf{H}_{\mathbf{g}_{k}}^{\mathrm{T}}\left[\begin{array}{cc}
0 & 1 \\
-1 & 0
\end{array}\right] \mathbf{H}_{\mathbf{g}_{k}}\right\} \\
\mathbf{A}_{1}=-2 \sum_{\mathrm{P}_{k}}\left\{\Delta m_{\mathrm{P}_{k}} \mathbf{H}_{\mathbf{h}_{k}}^{\mathrm{T}} \mathbf{T}\left(\vartheta_{\mathrm{P}_{k}}\right)\left[\begin{array}{cc}
0 & 1 \\
-1 & 0
\end{array}\right] \mathbf{T}^{\mathrm{T}}\left(\vartheta_{\mathrm{P}_{k}}\right) \mathbf{H}_{\mathbf{h}_{k}}\right\} \\
\mathbf{N}=\sum_{\mathrm{P}_{k}}\left\{\Delta R_{\mathrm{P}_{k}} \widehat{N}_{\mathrm{P}_{k}} \mathbf{H}_{\mathbf{m}_{k}}^{\mathrm{T}} \widetilde{\mathbf{T}}\left(\vartheta_{\mathrm{P}_{k}}\right) \mathbf{H}_{\mathbf{m}_{k}}\right\} \quad, \quad \widehat{N}_{\mathrm{P}_{k}}=\left.\left\{\sum_{j=1}^{J} \Delta m_{j} R_{j}\right\}\right|_{R_{1}=R_{P_{k}}}
\end{gathered}
$$




$$
\begin{gathered}
\mathbf{A}_{2}=-\sum_{\mathrm{P}_{k}}\left\{\Delta m_{\mathrm{P}_{k}} \mathbf{H}_{\mathbf{h}_{k}}^{\mathrm{T}} \mathbf{T}\left(\vartheta_{\mathrm{P}_{k}}\right)\left[\begin{array}{ll}
1 & 0 \\
0 & 1
\end{array}\right] \mathbf{T}^{\mathrm{T}}\left(\vartheta_{\mathrm{P}_{k}}\right) \mathbf{H}_{\mathbf{h}_{k}}\right\} \\
\mathbf{A}_{3}=-\sum_{\mathrm{P}_{k}}\left\{\Delta m_{\mathrm{P}_{k}} \mathbf{H}_{\mathbf{h}_{k}}^{\mathrm{T}} \mathbf{T}\left(\vartheta_{\mathrm{P}_{k}}\right)\left[\begin{array}{cc}
0 & 1 \\
-1 & 0
\end{array}\right] \mathbf{T}^{\mathrm{T}}\left(\vartheta_{\mathrm{P}_{k}}\right) \mathbf{H}_{\mathbf{h}_{k}}\right\} \\
\mathbf{a}_{0}\left(\Omega^{2}, \dot{\Omega}\right)=\sum_{\mathrm{P}_{k}}\left\{\Delta m_{\mathrm{P}_{k}} \mathbf{H}_{\mathbf{h}_{k}}^{\mathrm{T}} \mathbf{T}\left(\vartheta_{\mathrm{P}_{k}}\right)\left[\begin{array}{l}
R_{\mathrm{P}_{k}} \Omega^{2} \\
-R_{\mathrm{P}_{k}} \dot{\Omega}
\end{array}\right]\right\}
\end{gathered}
$$

\subsubsection{Campbell Diagrams}

The undamped natural frequencies and mode shapes at a given speed are found by setting $\dot{\Omega}=0$ and solving the eigenvalue problem associated with the homogeneous form of Eq. (37):

$\ddot{\mathbf{q}}+\left\{\mathbf{C}+\Omega\left(\mathbf{G}+\mathbf{A}_{1}\right)\right\} \dot{\mathbf{q}}+\left\{\mathbf{D}+\Omega^{2}\left(\mathbf{N}+\mathbf{A}_{2}\right)\right\} \mathbf{q}=\mathbf{0}$

In order to solve the eigenproblem, the state space format of Eq. (44) is used:

$$
\left[\begin{array}{cc}
\mathbf{I}_{H} & \mathbf{0} \\
\mathbf{0} & \mathbf{I}_{H}
\end{array}\right] \frac{\mathrm{d}}{\mathrm{d} t}\left[\begin{array}{c}
\mathbf{q}^{\mathrm{T}} \\
\dot{\mathbf{q}}^{\mathrm{T}}
\end{array}\right]+\left[\begin{array}{cc}
\mathbf{0} & -\mathbf{I}_{H} \\
\left\{\mathbf{D}+\Omega^{2}\left(\mathbf{N}+\mathbf{A}_{2}\right)\right\} & \left\{\mathbf{C}+\Omega\left(\mathbf{G}+\mathbf{A}_{1}\right)\right\}
\end{array}\right]\left[\begin{array}{c}
\mathbf{q}^{\mathrm{T}} \\
\dot{\mathbf{q}}^{\mathrm{T}}
\end{array}\right]=\mathbf{0}
$$

or $\quad \mathbf{I}_{2 H} \dot{\mathbf{s}}+\mathbf{B s}=\mathbf{0}$

where the state vector $\mathbf{s}=\left[\begin{array}{ll}\mathbf{q}^{\mathrm{T}} & \dot{\mathbf{q}}^{\mathrm{T}}\end{array}\right]^{\mathrm{T}}$ and $\mathbf{I}_{H}$ is an $H$-square identity matrix.

Let $\mathbf{s}=\mathbf{c e}^{\lambda t}$ and substituting in Eq. (45): 


$$
\left(\lambda \mathbf{I}_{2 H}+\mathbf{B}\right) \mathbf{c}=\mathbf{0}
$$

The above eigenvalue problem is solved for a given speed using the eig.m function in Matlab [42]. Using a range of speeds, the natural frequencies are calculated at each speed (from the imaginary part of the eigenvalues $\lambda$ ) and the Campbell diagram is plotted.

\subsubsection{Forced Response}

The system in Eq. (37) is cast in state-space format

$$
\dot{\mathbf{s}}=\chi(\mathbf{s}, t)
$$

where $\mathbf{s}=\left[\begin{array}{ll}\mathbf{q}^{\mathrm{T}} & \dot{\mathbf{q}}^{\mathrm{T}}\end{array}\right]^{\mathrm{T}}$ and the vector function $\boldsymbol{\chi}$ is

$$
\boldsymbol{\chi}=\left[\begin{array}{cc}
\mathbf{0} & \mathbf{I}_{H} \\
-\left\{\mathbf{D}+\Omega^{2}\left(\mathbf{N}+\mathbf{A}_{2}\right)+\dot{\Omega} \mathbf{A}_{3}\right\} & -\left\{\mathbf{C}+\Omega\left(\mathbf{G}+\mathbf{A}_{1}\right)\right\}
\end{array}\right] \mathbf{s}+\mathbf{H}_{\mathbf{f}}^{\mathrm{T}} \mathbf{f}(t)+\mathbf{a}_{0}\left(\Omega^{2}, \dot{\Omega}\right)
$$

The time domain response to a prescribed excitation $\mathbf{f}(t)$ (e.g. travelling wave chirp signal [42]), under arbitrary time-varying speed condition $\Omega(t)$, is then found by solving Eq. (47) using any one of the numerical integration routines in the ode suite of Matlab [46]. The

dynamic response in the rotating $X Y Z$ frame (Fig. 1) is then given by Eq. (2) i.e. $\Delta \mathbf{u}^{\text {(def) }}$. From this, the absolute coordinates of any subset of FE nodes can be determined using Eq. (1). For use in the BTT simulator of $[42,45]$, Eq. (47) is solved in Simulink and used in conjunction with Eqs. (1), (2), and the Hit Crossing block, to determine the blade tip timing values (as done previously with the state-space form of Eq. (3) [42]).

\subsection{Inclusion of Shaft Deflection - Extension to Shaft-Blisk System}

The effect of the deflection of point Q in Figure 1 is now considered. Let Q move to a point $\mathrm{Q}^{\prime}$. The position vector of the deformed position $\mathrm{P}^{\prime}$ of $\mathrm{P}(\mathrm{Eq}$. (9)) is now rewritten as: 


$$
\begin{gathered}
\mathbf{r}(t)=X_{\mathrm{Q}^{\prime}}^{(\mathrm{in})}(t) \mathbf{i}^{(\mathrm{in})}+Y_{\mathrm{Q}^{\prime}}^{(\mathrm{in})}(t) \mathbf{j}^{(\mathrm{in})}+\left(R+u(t)-u_{\mathrm{Q}^{\prime}}(t)\right) \mathbf{i}^{\prime}+\left(v(t)-v_{\mathrm{Q}^{\prime}}(t)\right) \mathbf{j}^{\prime} \\
+\left(Z_{0}+z(t)\right) \mathbf{k}
\end{gathered}
$$

where

$$
X_{\mathrm{Q}^{\prime}}^{(\mathrm{in})}(t) \mathbf{i}^{(\mathrm{in})}+Y_{\mathrm{Q}^{\prime}}^{(\mathrm{in})}(t) \mathbf{j}^{(\mathrm{in})} \equiv u_{\mathrm{Q}^{\prime}}(t) \mathbf{i}^{\prime}+v_{\mathrm{Q}^{\prime}}(t) \mathbf{j}^{\prime}
$$

is the transverse component of the position vector of $\mathrm{Q}^{\prime}$, and hence the transverse deflection of $\mathrm{Q}$. The position vector $\mathbf{r}(t)$ of $\mathrm{P}^{\prime}$ in Eq. (48) can be written as

$$
\mathbf{r}(t)=X^{(\mathrm{in})}(t) \mathbf{i}^{(\mathrm{in})}+Y^{(\mathrm{in})}(t) \mathbf{j}^{(\mathrm{in})}+\left(Z_{0}+z(t)\right) \mathbf{k}
$$

where the absolute coordinates $X^{(\mathrm{in})}(t), Y^{(\mathrm{in})}(t)$ of the deformed position of $\mathrm{P}, \mathrm{P}^{\prime}$ are

$$
\left[\begin{array}{l}
X^{(\mathrm{in})}(t) \\
Y^{(\mathrm{in})}(t)
\end{array}\right]=\left[\begin{array}{l}
X_{\mathrm{Q}^{\prime}}^{(\mathrm{in})}(t) \\
Y_{\mathrm{Q}^{\prime}}^{(\mathrm{in})}(t)
\end{array}\right]+\mathbf{T}(\varphi(t))\left[\begin{array}{l}
R \cos \vartheta \\
R \sin \vartheta
\end{array}\right]+\mathbf{T}(\varphi(t))\left[\begin{array}{l}
x(t)-x_{\mathrm{Q}^{\prime}}(t) \\
y(t)-y_{\mathrm{Q}^{\prime}}(t)
\end{array}\right]
$$

The modal matrices of the shaft-blisk system at zero-speed are used for transformation to the modal coordinate vector $\mathbf{q}(t)$ :

$$
\begin{gathered}
{\left[\begin{array}{l}
X_{\mathrm{Q}^{\prime}}^{(\mathrm{in})}(t) \\
Y_{\mathrm{Q}^{\prime}}^{(\mathrm{in})}(t)
\end{array}\right]=\left[\begin{array}{lll}
\psi_{x_{\mathrm{Q}}}^{(1)} & \cdots & \psi_{x_{\mathrm{Q}}}^{(H)} \\
\psi_{y_{\mathrm{Q}}}^{(1)} & \cdots & \psi_{y_{\mathrm{Q}}}^{(H)}
\end{array}\right] \mathbf{q}(t)} \\
{\left[\begin{array}{l}
x(t)-x_{\mathrm{Q}^{\prime}}(t) \\
y(t)-y_{\mathrm{Q}^{\prime}}(t)
\end{array}\right]=\left\{\left[\begin{array}{lll}
\psi_{x}^{(1)} & \cdots & \psi_{x}^{(H)} \\
\psi_{y}^{(1)} & \cdots & \psi_{y}^{(H)}
\end{array}\right]-\left[\begin{array}{lll}
\psi_{x_{\mathrm{Q}}}^{(1)} & \cdots & \psi_{x_{\mathrm{Q}}}^{(H)} \\
\psi_{y_{\mathrm{Q}}}^{(1)} & \cdots & \psi_{y_{\mathrm{Q}}}^{(H)}
\end{array}\right]\right\} \mathbf{q}(t)}
\end{gathered}
$$

In Eq. (50), the transformation matrix $\mathbf{T}$ is defined in Eq. $(6 \mathrm{c})$ and $\varphi(t)$ is the rigid rotation. Hence, the simulator equation (1) is extended to a shaft-blisk system as follows 


$$
\Delta \mathbf{u}^{(\mathrm{def})}(t)=\mathbf{T}_{\text {aug }}^{-1}(\varphi(t)) \mathbf{H}_{\mathrm{Q}} \mathbf{q}(t)+\left(\mathbf{H}_{\mathrm{P}}-\mathbf{H}_{\mathrm{Q}}\right) \mathbf{q}(t)
$$

$\mathbf{H}_{\mathrm{P}}$, as before, is the modal matrix for points on the blisk, and $\mathbf{H}_{\mathrm{Q}}$ the modal matrix of their companion points on the shaft centreline (as per Figure 1), both matrices evaluated from modal analysis of the system at zero speed with the blisk in its reference angular position.

The above equations mean that the following two modifications A, B to the analysis of the previous sections are necessary to extend it shaft-blisk systems.

A. The expression for $\mathbf{H}_{\mathbf{h}_{k}}$ in Eq. (15) is replaced by the following

$$
\mathbf{H}_{\mathbf{h}_{k}}=\left[\begin{array}{lll}
\psi_{x_{\mathrm{P}_{k}}}^{(1)} & \cdots & \psi_{x_{\mathrm{P}_{k}}}^{(H)} \\
\psi_{y_{\mathrm{P}_{k}}}^{(1)} & \cdots & \psi_{y_{\mathrm{P}_{k}}}^{(H)}
\end{array}\right]-\left[\begin{array}{lll}
\psi_{x_{\mathrm{Q}_{k}}}^{(1)} & \cdots & \psi_{x_{\mathrm{Q}_{k}}}^{(H)} \\
\psi_{y_{\mathrm{Q}_{k}}}^{(1)} & \cdots & \psi_{y_{\mathrm{Q}_{k}}}^{(H)}
\end{array}\right]
$$

where $\mathrm{Q}_{k}$ is the shaft centreline counterpart to point $\mathrm{P}_{k}$ on the blisk (see Figure 2; note that there will be multiple points $Q_{k}$ all coinciding with the same point on the shaft centreline, as many as there are points $\mathrm{P}_{k}$ in a given plane normal to shaft centreline).

B. A modal parametric excitation term $\boldsymbol{\rho}$ is added to Eqs. (8) and (37):

$$
\begin{aligned}
& \boldsymbol{\rho}(\varphi(t), \mathbf{q}, \dot{\mathbf{q}}, \ddot{\mathbf{q}}, \Omega, \dot{\Omega})= \\
& \Delta \mathbf{M}(\varphi(t)) \ddot{\mathbf{q}}+\Omega \cdot \Delta \mathbf{A}_{1}(\varphi(t)) \dot{\mathbf{q}}+\Omega^{2} \cdot \Delta \mathbf{A}_{2}(\varphi(t)) \mathbf{q}+\dot{\Omega} \cdot \Delta \mathbf{A}_{3}(\varphi(t)) \mathbf{q}+\Delta \mathbf{a}_{0}\left(\varphi(t), \Omega^{2}, \Omega\right)
\end{aligned}
$$

where $\Delta \mathbf{M}, \Delta \mathbf{A}_{1}, \Delta \mathbf{A}_{2}, \Delta \mathbf{A}_{3}, \Delta \mathbf{a}_{0}$ are time-varying matrices.

Modification A is evident by comparing the last three terms in Eq. (48) to Eq. (9). Modification B is the result of the first term in Eq. (48), which means that the system now has 
a deformable part that does not rotate (this part is defined by the collective of points $\mathrm{Q}$ originally along $\mathrm{OZ}$ in Figure 1).

Adding Eq. (55) to Eq. (37), results in the following equations of motion for the shaft-blisk system:

$$
\begin{aligned}
\ddot{\mathbf{q}}+\left\{\mathbf{C}+\Omega\left(\mathbf{G}+\mathbf{A}_{1}\right)\right\} \dot{\mathbf{q}}+\left\{\mathbf{D}+\Omega^{2}\left(\mathbf{N}+\mathbf{A}_{2}\right)+\dot{\Omega} \mathbf{A}_{3}\right\} \mathbf{q} \\
=\mathbf{H}_{\mathbf{f}}^{\mathrm{T}} \mathbf{f}(t)+\mathbf{a}_{0}\left(\Omega^{2}, \dot{\Omega}\right)+\boldsymbol{\rho}(\varphi(t), \mathbf{q}, \dot{\mathbf{q}}, \ddot{\mathbf{q}}, \Omega, \dot{\Omega})
\end{aligned}
$$

where all modal parameters belong to the shaft-blisk system at zero speed. In other works on shaft-blisk systems e.g. Santos et al. [44], the first four terms of $\boldsymbol{\rho}$ in Eq. (55) are incorporated with the corresponding terms on the left hand side of the equations of motion, rather than collected on the right hand side as above. The derivation of expressions for the terms of $\boldsymbol{\rho}$ is outside the scope of this paper.

$\boldsymbol{\rho}$ is only zero for $\varphi, \Omega, \dot{\Omega}=0$. For $\Omega$ constant (i.e. $\dot{\Omega}=0$ ), $\boldsymbol{\rho}$ is periodic and introduces parametric excitation into the system. In this condition, the coefficients of $\mathbf{q}, \dot{\mathbf{q}}, \ddot{\mathbf{q}}$ in Eq. (56) are not constant matrices and eigenvalue analysis for the Campbell diagrams cannot be performed since this requires constant matrices. However, in this paper, the parametric excitation effect is not considered. Hence, the eigenvalue analysis for Campbell diagrams for the rotor-blisk system is performed at any fixed speed $\Omega$ as before (section 2.4.1), with modification A (above) but with the parametric term $\boldsymbol{\rho}$ omitted. The same thing was done by Ma et al. [2] and Chun and Lee [18], who did eigenvalue analysis of the rotor-blisk system equations but did not declare the omission of any periodically varying terms in the matrices (in fact, there is no mention of parametric excitation in these papers). The Campbell diagrams so generated in these references $[2,18]$ are indeed verified by the present analysis 
(sections 3.4.2 and 3.5). Forced vibration analysis is done as in section 2.4.2 merely to crosscheck the resulting resonances with the results of the Campbell diagram.

If $\boldsymbol{\rho}$ is not omitted, rather than generating Campbell diagrams via eigenvalue analysis, one generates waterfall diagrams (WDs) by integrating for the time domain responses of Eq. (56) over a range of fixed speeds $\Omega$ and then taking their Fast Fourier Transforms (FFTs), as done by Santos et al. [44]. The researchers [44] considered a simple model of a rotor-blades assembly, where the rotor was mounted with lateral flexibility to simulate shaft deflection. The external excitation consisted of a rotating unbalance (via term $\Delta \mathbf{a}_{0}$ in Eq. (55)) and impulses applied to either a blade or the rotor. The WDs all contained frequencies $\Omega$. The WDs of the rotor lateral vibration (in an inertial coordinate frame, Figures 5, 7 in reference [44]) additionally showed frequencies of the form $\omega_{n} \pm \Omega$, where $\omega_{n}$ is one of the natural frequencies of the shaft-blades system at zero speed. Such parametric vibration frequencies were not evident in the WDs of the blades response (in a rotating coordinate frame, Figures 6, 8 in reference [44]), which showed variation in one of the natural frequencies that appears to be similar to the variation on a Campbell diagram obtained as discussed above (i.e. excluding

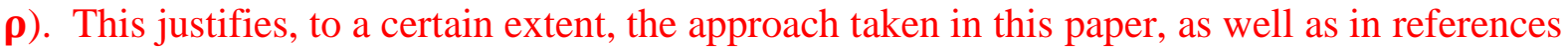
$[2,18]$. It should be noted that the researchers [44] also considered gravitational forces, as well as nonlinearity (which are not considered here).

\section{VALIDATION}

This section presents a number of examples of shaft-disk-blades assemblies considering one or more of the rotational effects. All assemblies are modelled and analysed using the method presented in the previous section, and the results are correlated against analytical or experimental results from the literature, or results from commercial FE software. 
The examples considered are presented in Table 1. Example \#0 is not actually about rotational effects, but about the effective stiffness variation induced by a longitudinal load on a beam. It therefore serves as a preliminary verification of the accuracy of the original approach introduced in section 2.2.2 for considering the centrifugal stiffening effect. For examples \#3a, \#3b and \#4, which are shaft-blisk systems, the parametric excitation effect is omitted, as per last two paragraphs of section 2.5.

Table 1: Examples used for model validation

\begin{tabular}{ccc}
\hline Example & Effects considered & Validation Source \\
\hline 0 & Longitudinal load on beam & Shaker, 1975 [47] \\
& (preliminary check for Example 1) & \\
\hline 1 & Centrifugal Stiffening & Chen et al., 2016 [39] \\
\hline 2 & Coriolis forces & Ruffini et al, 2015 [32] \\
\hline $3 a$ & Gyroscopic moment - effect of disk & ANSYS FE software \\
& Gyroscopic moment - example from & Chun and Lee, 1996 [18]/ \\
& literature & Zhang et al, 1994 [48] \\
\hline $3 b$ & Gyroscopic, Coriolis, Spin Softening, & Ma et al, 2015 [2] \\
\hline 4 & Centrifugal Stiffening & \\
\hline
\end{tabular}

3.1 Example \# 0 - Longitudinal Load on Beam (Table 1, preliminary check for section 2.2.2)

Fig. 5 shows a rectangular-section cantilever beam $(b=5 h)$ subjected to a constant longitudinal force. Using an analytical approach, Shaker [47] calculated the natural frequencies of the beam for a range of force values (tension and compression). 


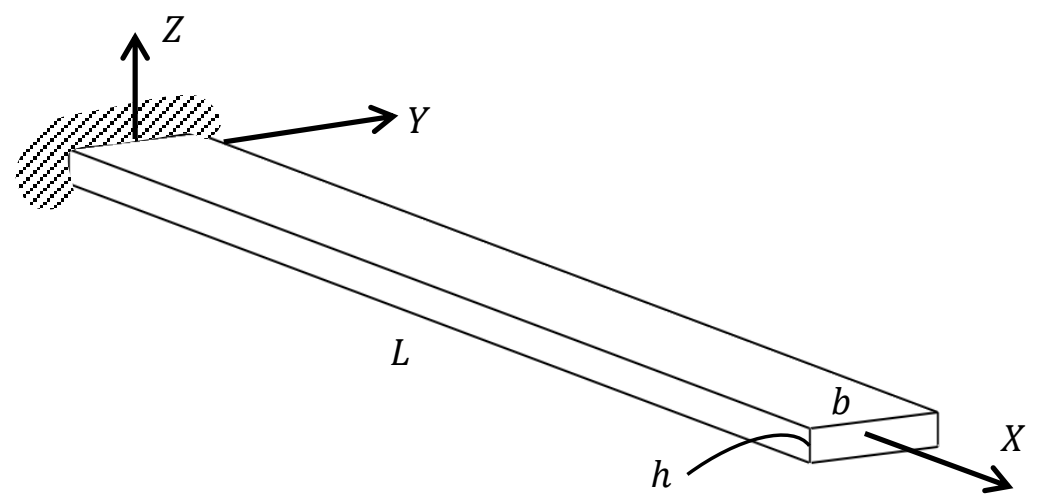

Fig. 5. Example 0 - Cantilever beam subjected to axial load

With the proposed method, for an applied load of $Q(Q>0$ for compression), Eqs. (44) and $(40 \mathrm{a}, \mathrm{b})$ are modified as follows:

$$
\begin{gathered}
\ddot{\mathbf{q}}+\left\{\mathbf{D}+\mathbf{N}_{\mathrm{mod}}\right\} \mathbf{q}=\mathbf{0} \\
\mathbf{N}_{\text {mod }}=\sum_{\mathrm{P}_{k}}\left\{\Delta R_{\mathrm{P}_{k}} \widehat{N}_{\mathrm{P}_{k_{\text {mod }}}} \mathbf{H}_{\mathbf{m}_{k}}^{\mathrm{T}} \breve{\mathbf{T}}\left(\vartheta_{\mathrm{P}_{k}}\right) \mathbf{H}_{\mathbf{m}_{k}}\right\} \quad, \widehat{N}_{\mathrm{P}_{k_{\text {mod }}}}=-Q
\end{gathered}
$$

It is seen from Eqs. $(58 a, b)$ that Eqs. $(40 a, b)$ were modified to allow for a compressive load that is constant for all segments (and not varying as in centrifugal forces). 200 divisions (points $\mathrm{P}_{k}$ ) were used for accuracy. From Fig. 5 and Fig. 3, $\vartheta_{\mathrm{P}_{k}}=0$ in Eq. (21). The basis modes used in the matrices $\mathbf{D}$ and $\mathbf{H}_{\mathbf{m}_{k}}$ pertain to the undamped modes of the given cantilever at zero longitudinal load. The number of basis modes $=10$ i.e. 5 modes were used for each bending plane $X Z$ and $X Y$. The basis modes in each plane were calculated using exact formulas for natural circular frequencies and mass normalized mode shapes of the cantilever at zero longitudinal load (found in [49]), such that Eqs. (4), (5) are given by

$$
\mathbf{D}=\operatorname{diag}\left(\left[\begin{array}{lllll}
\varpi_{Y Y_{1}} & \varpi_{Z Z_{1}} & \varpi_{Y Y_{2}} & \varpi_{Z Z_{2}} & \cdots \cdots
\end{array}\right]\right)
$$




$$
\mathbf{H}_{\mathbf{m}_{k}}=\left[\begin{array}{ccccc}
0 & 0 & 0 & 0 & \cdots \cdots \\
-\frac{\mathrm{d} \phi_{Z}^{(1)}}{\mathrm{d} X} & 0 & -\frac{\mathrm{d} \phi_{Z}^{(2)}}{\mathrm{d} X} & 0 & \cdots \cdots \\
0 & \frac{\mathrm{d} \phi_{Y}^{(1)}}{\mathrm{d} X} & 0 & \frac{\mathrm{d} \phi_{Y}^{(2)}}{\mathrm{d} X} & \cdots \cdots
\end{array}\right]
$$

where $\varpi_{Y Y_{i}}, \phi_{Z}^{(i)}$ denote the natural circular frequency and translational mode shape for $i^{\text {th }}$ bending mode of the cantilever in the $X Z$ plane (i.e. bending about $Y Y$ neutral axis) at zero longitudinal load, and $\varpi_{Z Z_{i}}, \phi_{Y}^{(i)}$ denote similar quantities for the $X Y$ plane.

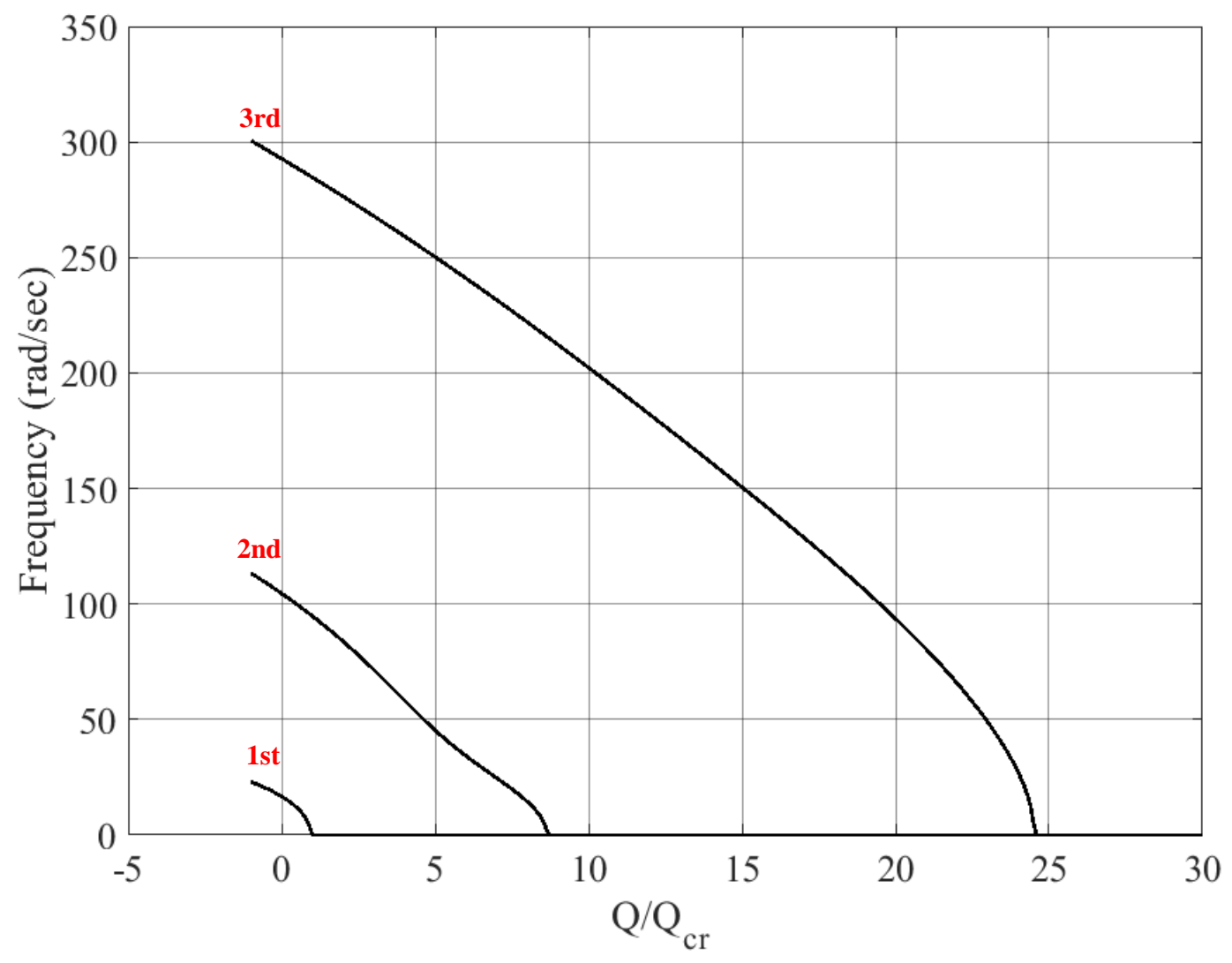

Fig. 6. Example 0 - natural frequencies vs axial load $(X Z-$ plane $)$ 


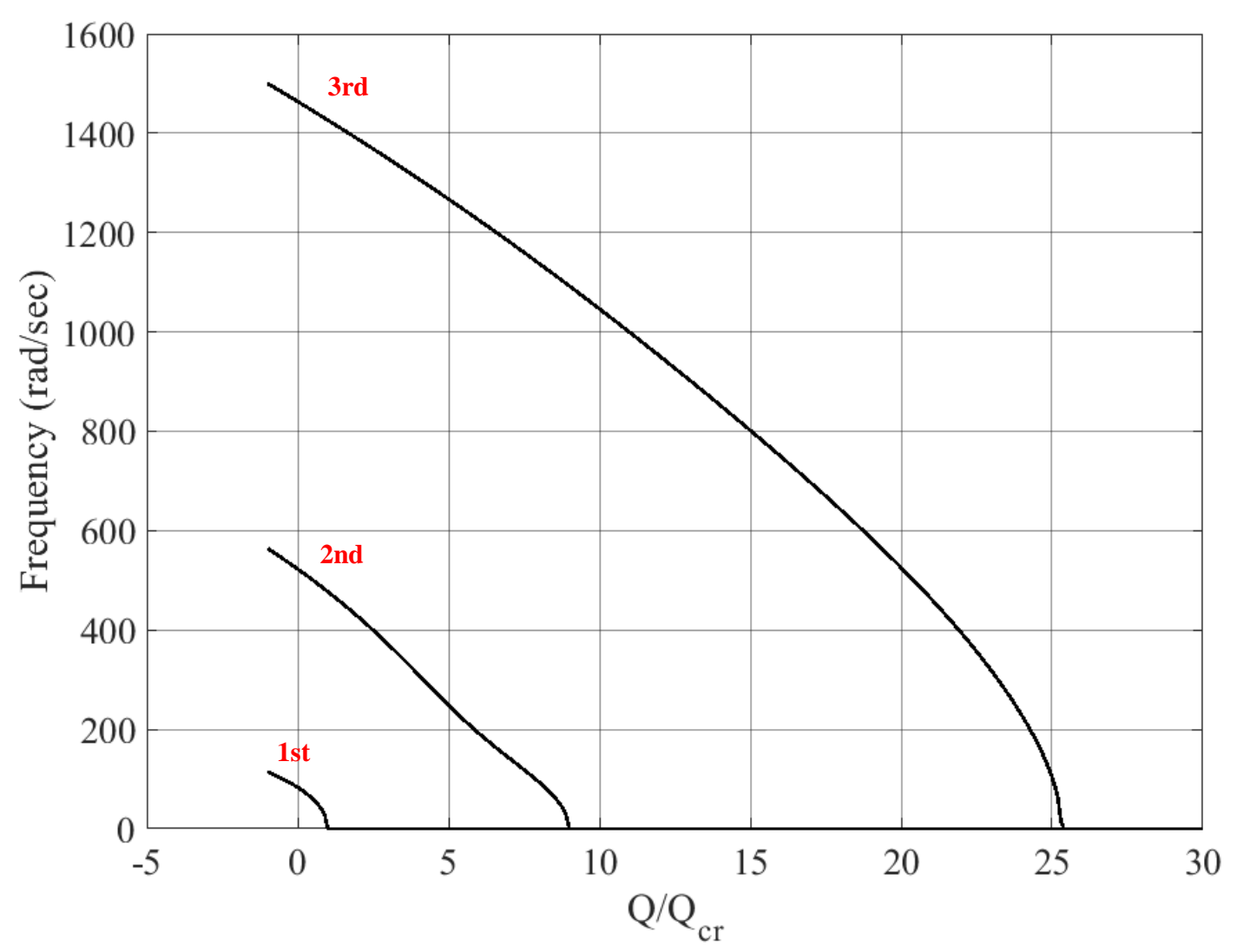

Fig. 7. Example 0 - natural frequencies vs axial load ( $X Y-$ plane $)$

The eigenvalue problem defined by Eq. (57) was then solved for each given value of $Q$ within a wide range. The eigenvalue problem yields natural frequencies for bending in each plane $X Z, X Y$. Fig. 6 shows the variation of the first three natural frequencies for bending in the $X Z$ plane with $Q / Q_{\mathrm{cr}_{Y Y}}$ and Fig. 7 shows the variation of the first three natural frequencies for bending in the $X Y$ plane with $Q / Q_{\mathrm{cr}_{Z Z}}$ and where $Q_{\mathrm{cr}_{Y Y}}, Q_{\mathrm{cr}_{Z Z}}$ respectively denote the first critical buckling loads of the cantilever in the $X Z, X Y$ planes:

$Q_{\mathrm{cr}_{Y Y, Z Z}}=\frac{\pi^{2} E I_{Y Y, Z Z}}{(2 L)^{2}}$

where $L$ is the length and $E I_{Y Y}, E I_{Z Z}$ denote the flexural rigidity in the $X Z, X Y$ planes respectively. As per theory in [50], for either plane, the first natural frequency goes to zero when $Q / Q_{\mathrm{cr}}=1$ (first critical buckling load), while the second one becomes zero at 
$Q / Q_{\mathrm{cr}}=9$ (second critical buckling load), and the third frequency goes to zero at $Q / Q_{\mathrm{cr}}=$ 25 (third critical buckling load). Fig. 8 shows the variation of the non-dimensional natural frequency $\lambda_{i}^{2}$ in $X Z$ plane $\left(\lambda_{i}^{2}=\omega_{Y Y_{i}} L^{2} \sqrt{\frac{m}{E I_{Y Y}}}\right.$ where $\omega_{Y Y_{i}}$ is the $i^{\text {th }}$ natural circular frequency in the $X Z$ plane under load and $m$ the mass per unit length). Fig. 8, as well as Fig. 6, and Fig. 7, show that the method presented in this paper accurately reproduces the analytical model results presented in [47].

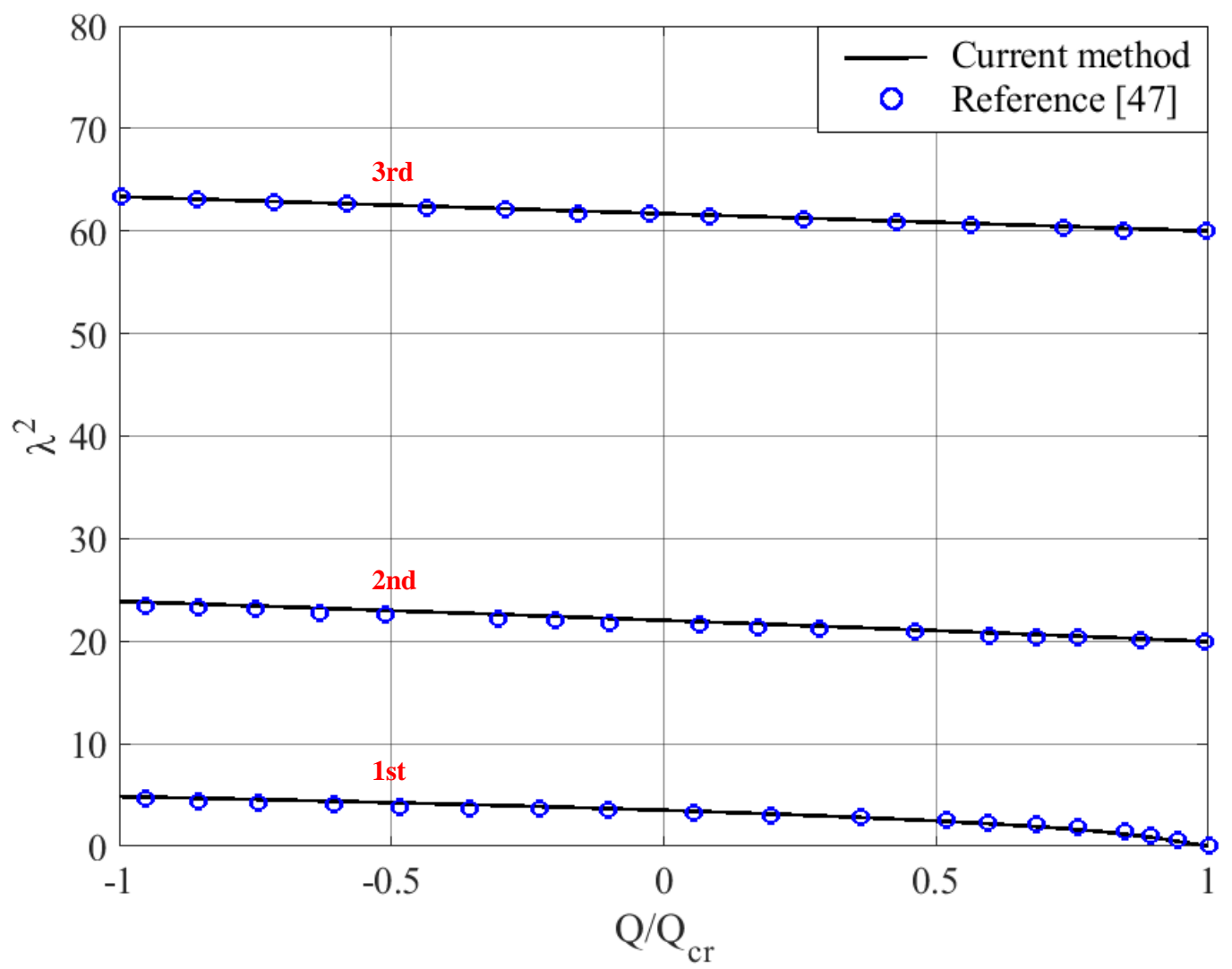

Fig. 8. Example 0 - non-dimensional frequencies of axially loaded cantilever beam (XZ-plane)

\subsection{Example \# 1 - Centrifugal Stiffening (Table 1)}

In this example, a simple blade model consisting of a cantilever beam of rectangular cross section (Fig. 9) is used to validate the ability of the present method to calculate the natural 
frequencies of the rotating beam under the effect of centrifugal stiffening. The results are compared to those obtained by Chen et al. [39] in 2016, who used a variational iteration method to study the flapwise vibration of rotating uniform Euler-Bernoulli beams. Nondimensional natural frequencies were calculated in [39] and so, the dimensions of the beam are assumed as shown in Fig. 9, and the material properties of the beam are assumed as follows: density $7800 \mathrm{~kg} / \mathrm{m}^{3}$, Young Modulus $210 \mathrm{GPa}$, and Poisson ratio 0.28.
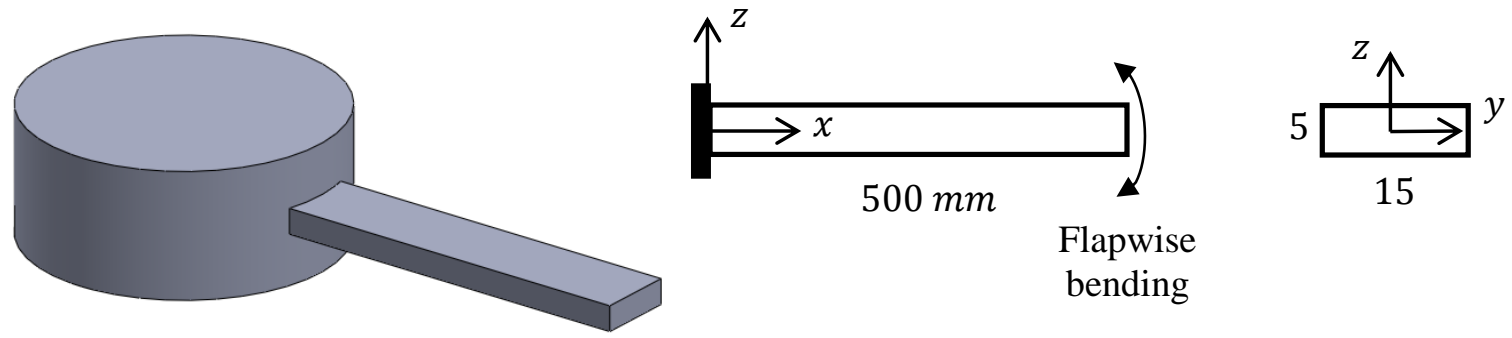

Fig. 9. Example 1 - Blade model used in [39]

Using the present method, the matrices $\mathbf{C}, \mathbf{G}, \mathbf{A}_{1}, \mathbf{A}_{2}$ in Eq. (44) were set to zero. The matrix $\mathbf{N}$ is as given by Eqs. $(40 \mathrm{a}, \mathrm{b})$ with $\vartheta_{\mathrm{P}_{k}}=0$ and the number of divisions (points $\mathrm{P}_{k}$ ) set to 500 (the hub radius was taken to be zero). Only the flapwise ( $X Z$ plane) modes were of interest since only these were considered in [39]. Hence, the matrices $\mathbf{D}$ and $\mathbf{H}_{\mathbf{m}_{k}}$ were a reduced form of Eqs. (50), (51) using only modes in the $X Z$ plane:

$$
\begin{gathered}
\mathbf{D}=\operatorname{diag}\left(\left[\begin{array}{lll}
\varpi_{Y Y_{1}} & \varpi_{Y Y_{2}} & \cdots \cdots
\end{array}\right]\right) \\
\mathbf{H}_{\mathbf{m}_{k}}=\left[\begin{array}{ccc}
0 & 0 & \cdots \cdots \\
-\frac{\mathrm{d} \phi_{Z}^{(1)}}{\mathrm{d} X} & -\frac{\mathrm{d} \phi_{Z}^{(2)}}{\mathrm{d} X} & \cdots \cdots \\
0 & 0 & \cdots \cdots
\end{array}\right]
\end{gathered}
$$


A total of $H=10$ modes were used in the above equations. The data in the matrices $\mathbf{D}$ and $\mathbf{H}_{\mathbf{m}_{k}}$, were evaluated using the exact formulas given in [49].

Following [39], the natural frequencies solutions from the eigenproblem of Eq. (44) were converted to non-dimensional natural frequencies $\widetilde{\omega}_{Y Y_{i}}$ corresponding to the non-dimensional rotational speed $\tilde{\Omega}$ :

$$
\widetilde{\omega}_{Y Y_{i}}=\omega_{Y Y_{i}} L^{2} \sqrt{\frac{m}{E I_{Y Y}}}, i=1,2 \ldots, \quad \tilde{\Omega}=\Omega L^{2} \sqrt{\frac{m}{E I_{Y Y}}}
$$

Table 2: Natural frequencies of blade model

\begin{tabular}{lllllllllll}
\hline \multirow{2}{*}{ Non-dim. } & \multicolumn{2}{l}{$1^{\text {st }}$ frequency } & \multicolumn{4}{c}{$2^{\text {nd }}$ frequency } & \multicolumn{5}{c}{$3^{\text {rd }}$ frequency } \\
\cline { 2 - 10 } & ref [39] & Present & \%error & ref [39] & Present & \%error & ref [39] & Present & \%error \\
\hline 0 & 3.516 & 3.516 & 0 & 22.0345 & 22.0345 & 0 & 61.6972 & 61.6972 & 0 \\
\hline 3 & 4.7973 & 4.8001 & 0.058 & 23.3203 & 23.325 & 0.020 & 62.985 & 62.9889 & 0.006 \\
\hline 6 & 7.3604 & 7.3672 & 0.092 & 26.8091 & 26.8273 & 0.068 & 66.684 & 66.7013 & 0.026 \\
\hline 12 & 13.1702 & 13.1873 & 0.130 & 37.6031 & 37.6728 & 0.185 & 79.6145 & 79.697 & 0.104 \\
\hline
\end{tabular}

Table 2 presents a comparison with the results published in [39], where good agreement is demonstrated (maximum error $0.19 \%$ ). The Campbell diagram of the first three flap-wise frequencies is presented in Fig. 10. The simulation time to generate this diagram was only 20 sec.

The above procedure was repeated using FE (with solid elements) to determine the zerospeed modal data in matrices $\mathbf{D}$ and $\mathbf{H}_{\mathbf{m}_{k}}$. The resulting Campbell diagram (Fig. 11) was virtually identical to that in Fig. 10, but took much longer to generate (35 min), time taken to find the closest FE nodes to each $\mathrm{P}_{k}$ point, in order to calculate angular displacement modal data from the FE translational displacement modal data for each of the 500 divisions using 
the method of section 2.3. If beam elements were used in the FE, rather than solid elements, the method of section 2.3 would not be required (since beam elements have angular degrees of freedom). Table 3 shows that the maximum discrepancy relative to [39] increases to $1 \%$ since solid elements do not use the Euler-Bernoulli model of [39].

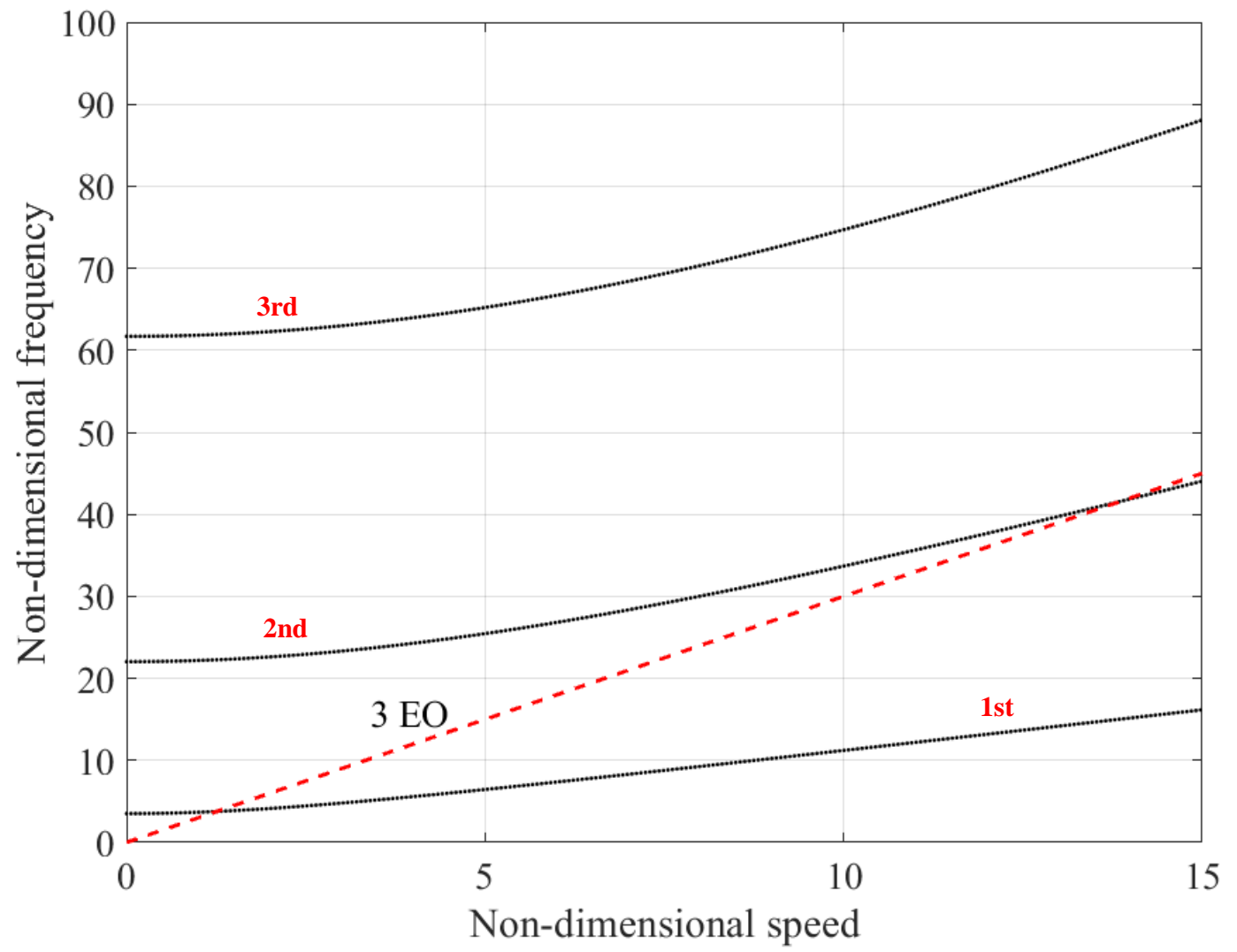

Fig. 10. Example 1 - Campbell diagram of blade model (using zero-speed modal data derived from analytical expressions)

Table 3: Natural frequencies of blade model (using zero-speed modal data from FE)

\begin{tabular}{llllllllll}
\hline \multirow{2}{*}{ Nondim. } & \multicolumn{2}{l}{$1^{\text {st }}$ frequency } & \multicolumn{3}{c}{$2^{\text {nd }}$ frequency } & \multicolumn{3}{c}{$3^{\text {rd }}$ frequency } \\
\cline { 2 - 10 } & Ref [39] & Present & \%error & Ref [39] & Present & \%error & Ref [39] & Present & \%error \\
\hline 0 & 3.516 & 3.5172 & 0.034 & 22.0345 & 21.9499 & -0.384 & 61.6972 & 61.0543 & -1.042 \\
\hline 3 & 4.7973 & 4.814 & 0.348 & 23.3203 & 23.2662 & -0.232 & 62.985 & 62.3351 & -1.032 \\
\hline 6 & 7.3604 & 7.3943 & 0.461 & 26.8091 & 26.845 & 0.134 & 66.684 & 66.0295 & -0.982 \\
\hline 12 & 13.1702 & 13.2362 & 0.501 & 37.6031 & 37.9795 & 1.001 & 79.6145 & 79.108 & -0.636 \\
\hline
\end{tabular}




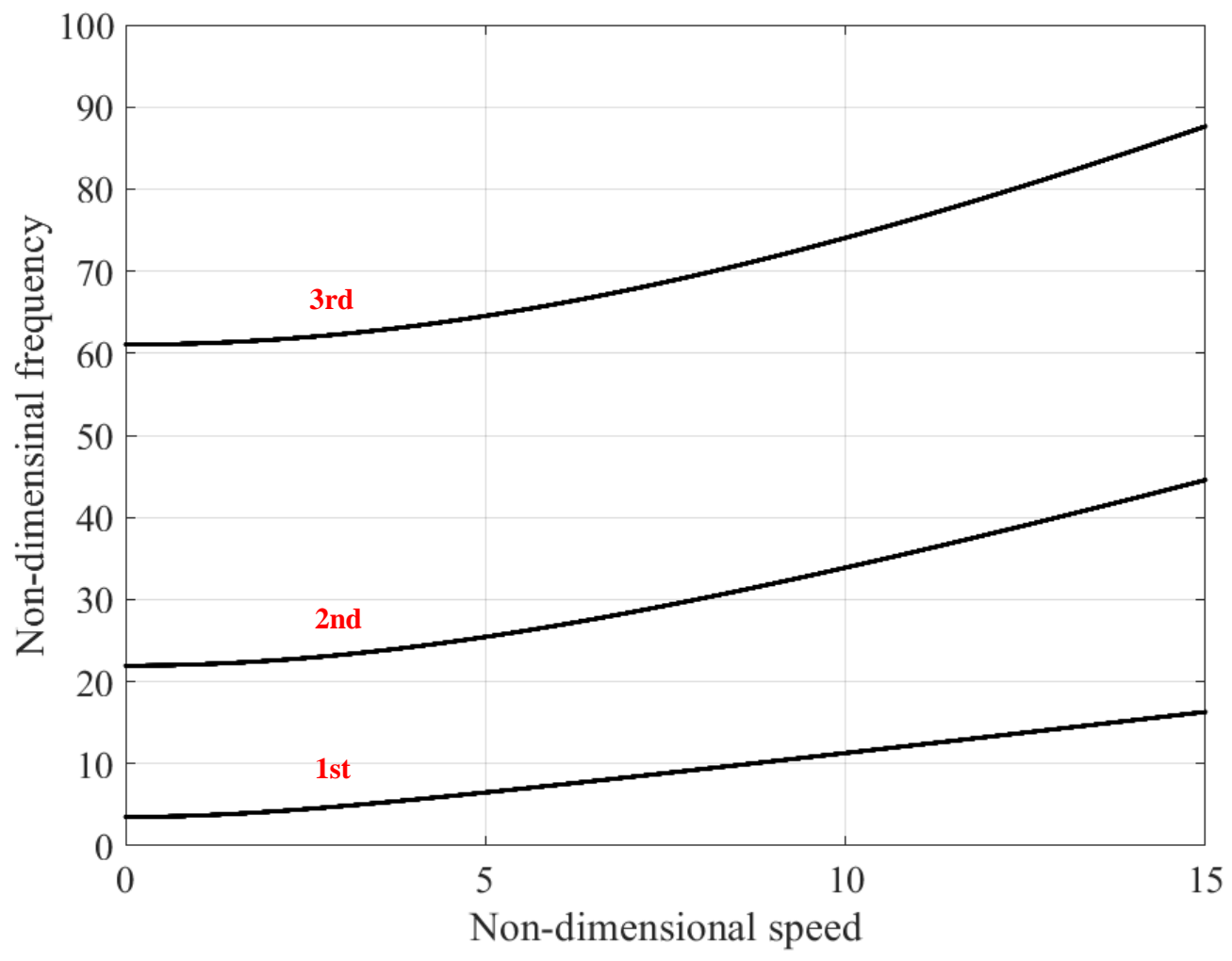

Fig. 11. Example 1 - Campbell diagram of blade model using zero-speed modal data derived from FE

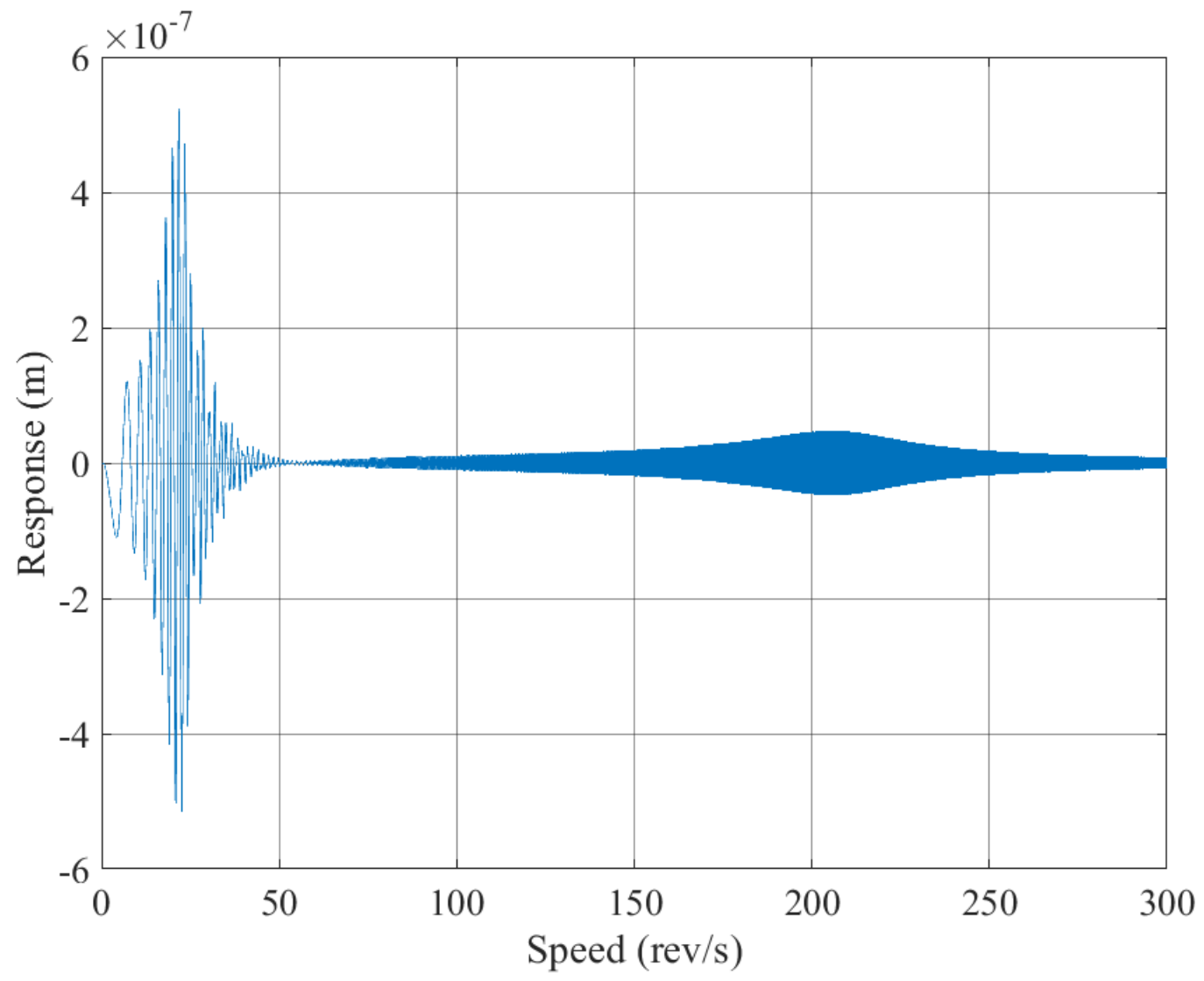

Fig. 12. Example 1 - forced response of blade model 
A chirp excitation force was applied at one of the free end nodes in the Z-direction under variable speed conditions such that the excitation frequency was a fixed ratio (Engine Order, EO) of the rotational speed $\Omega(t)$ :

$f(t)=A \sin \left[\mathrm{EO}\left(\Omega_{\mathrm{i}} t+\left(\frac{\Omega_{\mathrm{f}}-\Omega_{\mathrm{i}}}{2 T_{\mathrm{s}}}\right) t^{2}\right)\right]$

where $\Omega_{\mathrm{i}}, \Omega_{\mathrm{f}}$ are the values of the initial and final angular speeds over a duration $T_{\mathrm{s}}$. Fig. 12 shows the simulator response (as per section 2.4.2) for $A=1$ and $\mathrm{EO}=3$ (i.e. "3EO excitation") and $T_{\mathrm{s}}=3 \mathrm{sec}, \zeta_{r}=0.05$ (Eq. (5)), where it is seen that the resonance speeds agree with the results from Campbell diagram.

\subsection{Example \# 2 - Coriolis Forces (Table 1)}

Fig. 13 shows a blisk which was specially designed by Ruffini et al. [32] in 2015 for experimental and numerical (FE) analysis of Coriolis effects. It is modelled and analysed again here using the proposed method and the results are validated against the published results in [32].

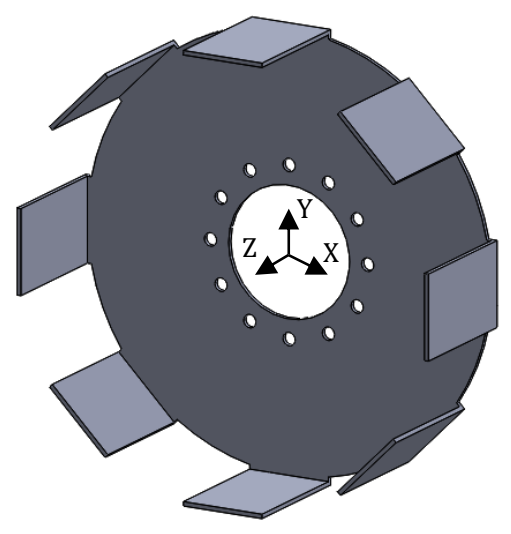

Fig. 13. Example 2: specially designed blisk for Coriolis effects validation, as used in [32] 
As shown in Fig. 13, the blades are bent $90^{\circ}$ (out-of-plane). According to authors of [32] this increased the Coriolis-induced frequency splits as much as possible in order to have distinct travelling wave modes. Such a design also minimised the effects of mistuning which could otherwise lead to frequency splits that overshadow the Coriolis effects. The horizontal leaning of the blades also makes centrifugal stiffening not noticeable [32].

Detailed dimensions and boundary conditions of the model were not available in [32], so a number of attempts have been made with different dimensions until accurate natural frequencies of the non-rotating blisk were obtained. The zero-speed modal data were obtained by ANSYS with the nodes along the periphery of the central hole and the small holes assumed fixed. The number of degrees of freedom used for zero-speed modal data computation was 662337.

In Eq. (44), all the matrices were set to zero except $D$ and $A_{1}$. The number of basis modes $H=10$. The number of rotational inertia divisions (points $\mathrm{P}_{k}$ ) used in this matrix (Eq. (39)) was 54. Each blade divided into a 3 divisions in axial direction (not radial, due to their bent), and the disk inertia was divided into 30 divisions [See Appendix A].

The Campbell diagram generated using the new method is shown in Fig. 14 for mode nos. 1 to 7 over the speed range $0-500 \mathrm{rpm}$. The simulation time was only $10 \mathrm{sec}$. The experimental results obtained in [32] are reproduced in Fig. 14 and good correlation with the predictions is evident, proving the validity of the new method. The critical speeds for single engine order are shown in Table 4.

Table 4: Critical speeds (current method)

\begin{tabular}{cccccc}
\hline Mode \# & 1 & 2 & 3 & 4 & 5 \\
\hline Critical speed (rev/s) & 148.9 & 156.8 & 174.2 & 192.15 & 258.7 \\
\hline
\end{tabular}




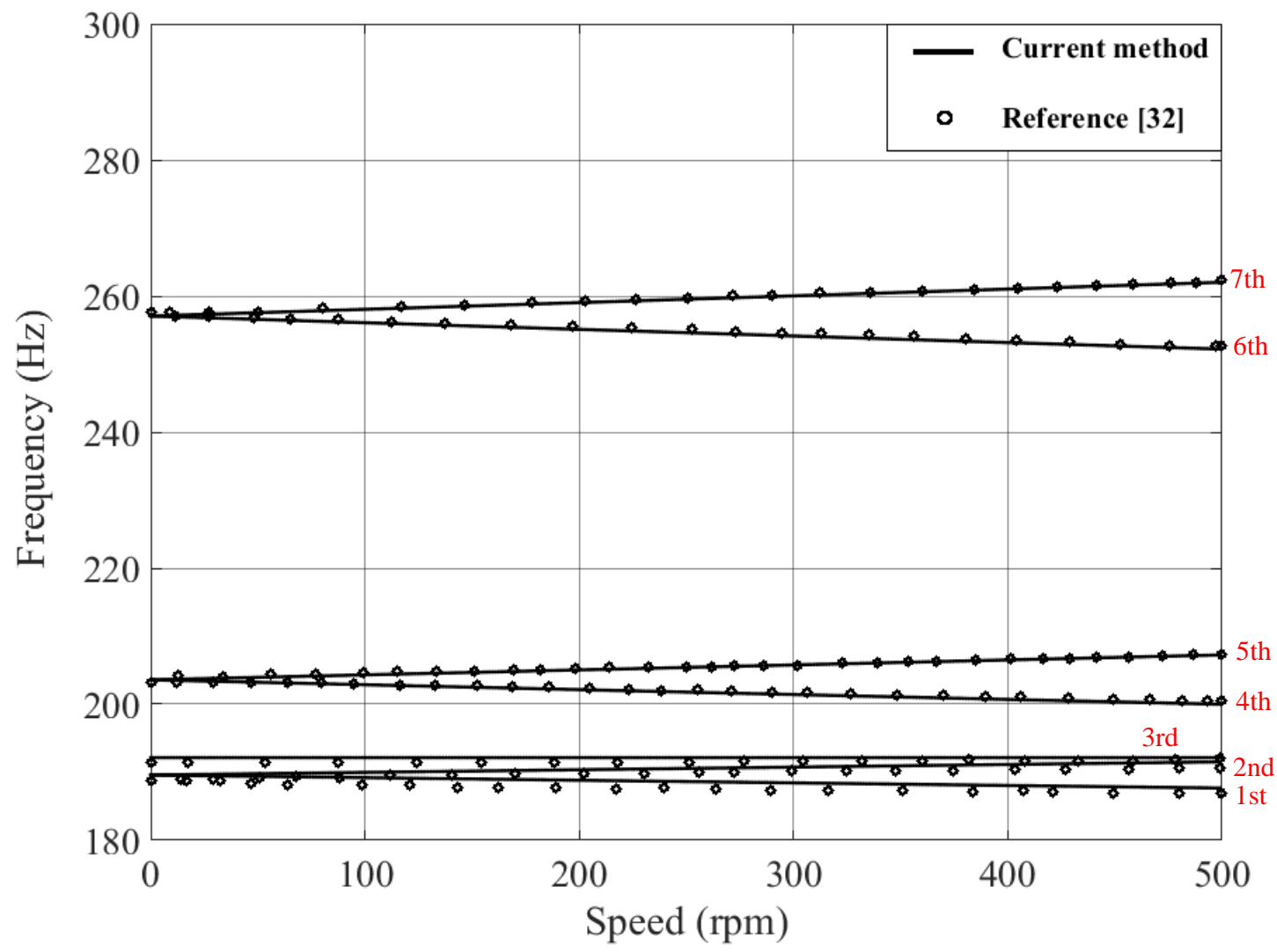

Fig. 14. Example 2 - Campbell diagram of the blisk specially designed for Coriolis effects

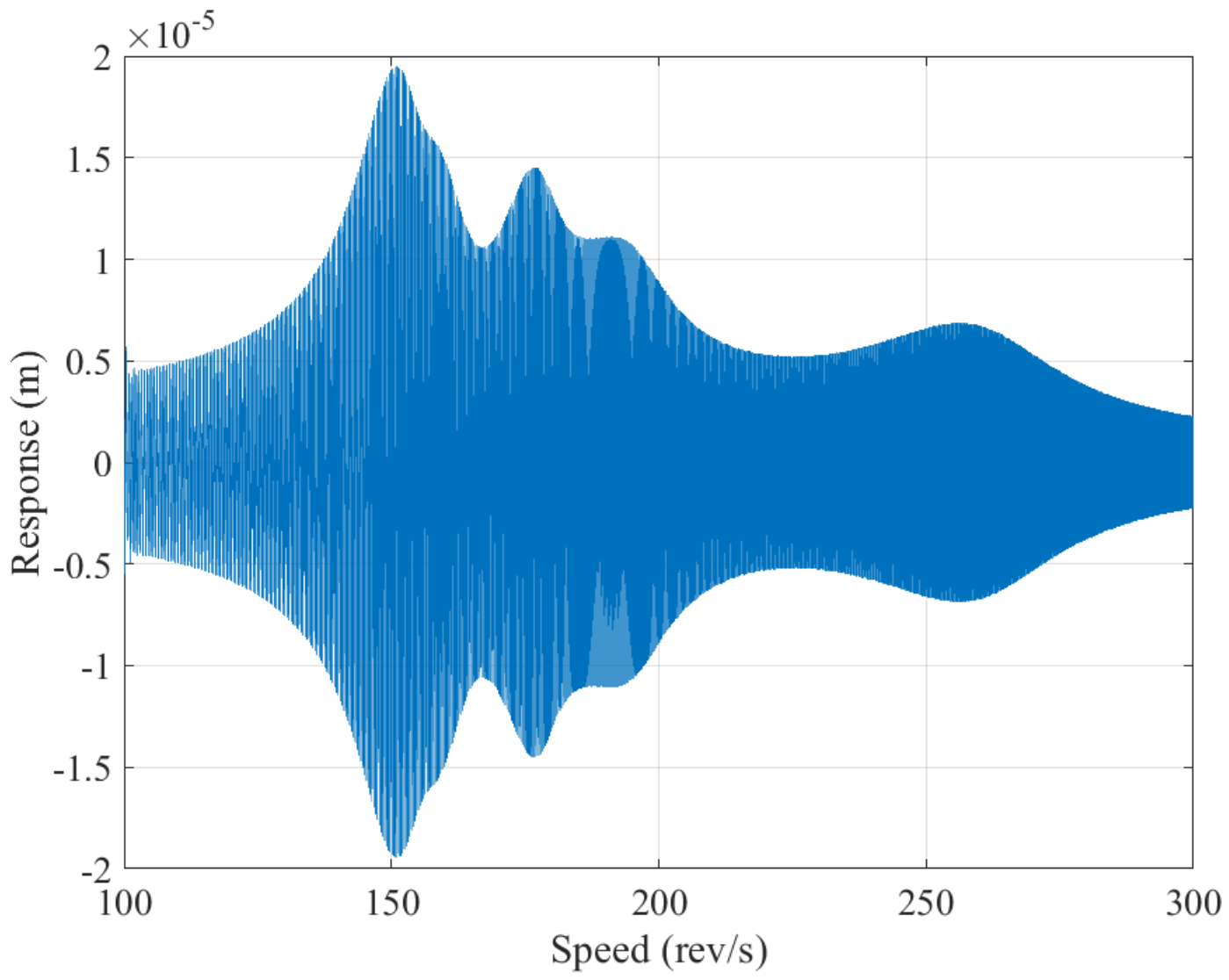

Fig. 15. Example 2 - forced response of the blisk specially designed for Coriolis effects 
A chirp excitation force was applied at one of the blade tips (normal to the blade) under variable speed condition, as per Eq. (56). Fig. 15 shows the simulator response (as per section 2.4.2) for $A=1$ and $\mathrm{EO}=1$ and $T_{\mathrm{s}}=4 \mathrm{sec}, \zeta_{r}=0.05$ (Eq. (5)). The resonance speeds agree with the Campbell diagram results with small percentages of errors since the response is transient and not strictly steady-state.

\subsection{Example \# 3a,b - Gyroscopic Effect}

3.4.1 Example \# 3a-Gyroscopic moment-effect of disk flexibility (Table 1)
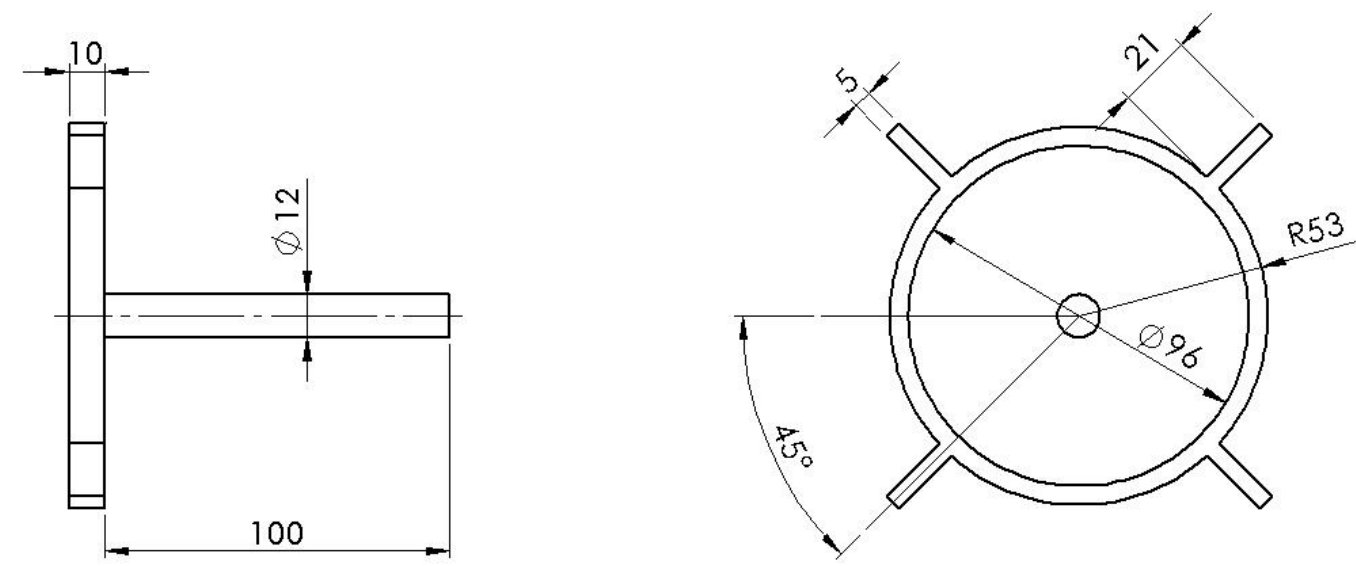

Fig. 16. Example 3a - blisk supported by free-fixed shaft

The model shown in Fig. 16 consists of a disk with 4 blades positioned at the end of a cantilevered shaft. It is therefore subjected to gyroscopic coupling which leads to splits in frequencies with increasing rotational speed. The model was created using SolidWorks and ANSYS, the material used was steel with $7850 \mathrm{~kg} / \mathrm{m}^{3}$ density and $200 \mathrm{GPa}$ elasticity modulus. The disk is considered bonded to the shaft, and the number of DOF used to generate the zero-speed modal data was 134217. 
The first five zero-speed natural frequencies and mode shapes are shown in Fig. 17 and Table 5. It is clear from Fig. 17 that modes 1, 2, 4, and 5 are bending modes, so they are more likely to be affected by gyroscopic moments (resulting in frequency splits).
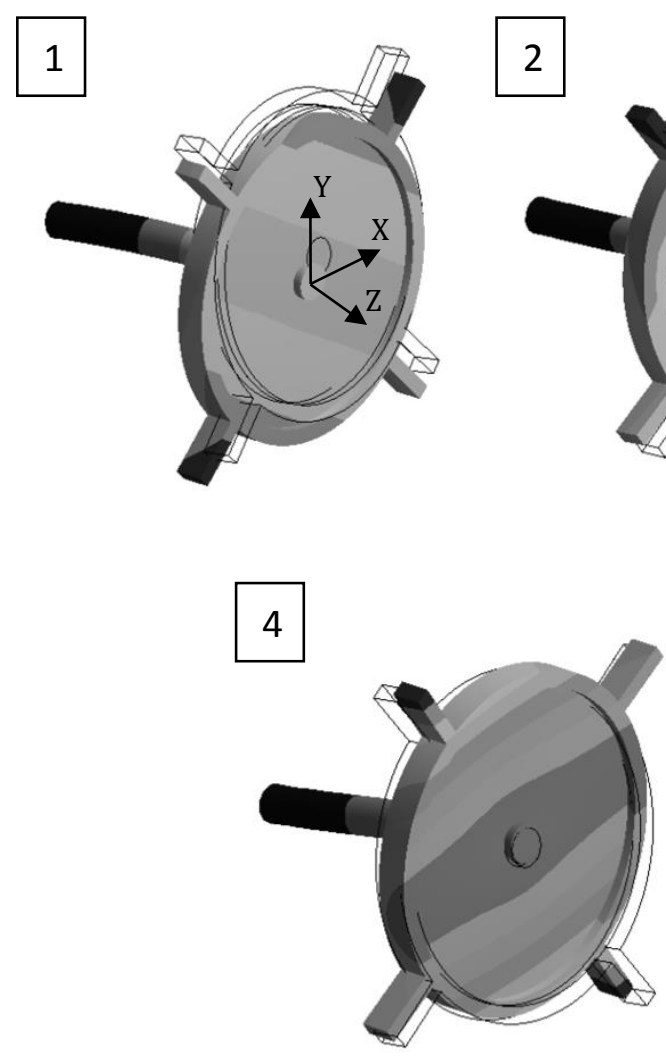
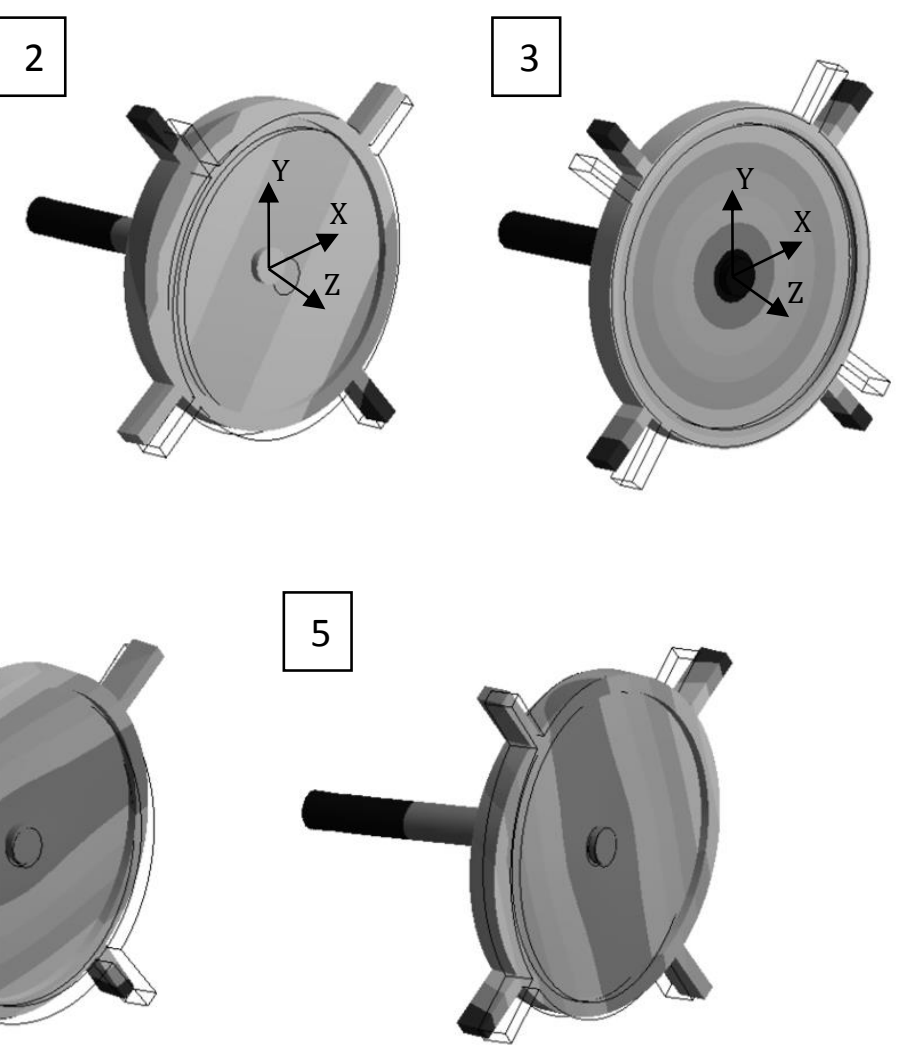

Fig. 17. Example 3a - zero-speed mode shapes of blisk supported by free-fixed shaft

Table 5: Natural frequencies blisk supported by free-fixed shaft at zero speed

\begin{tabular}{llllll}
\hline Mode \# & 1 & 2 & 3 & 4 & 5
\end{tabular}

Natural frequency (Hz)

171

171

231

611

611

Another modal analysis was carried out using the rotordynamics analysis facility in ANSYS over a rotational speed range $0-600 \mathrm{rev} / \mathrm{s}$, and the Campbell diagram was extracted as shown 
in Fig. 18. In this ANSYS analysis, the gyroscopic effect was activated on its own (without Coriolis and spin softening effects added) by applying the CORIOLIS command in a stationary reference frame [40].

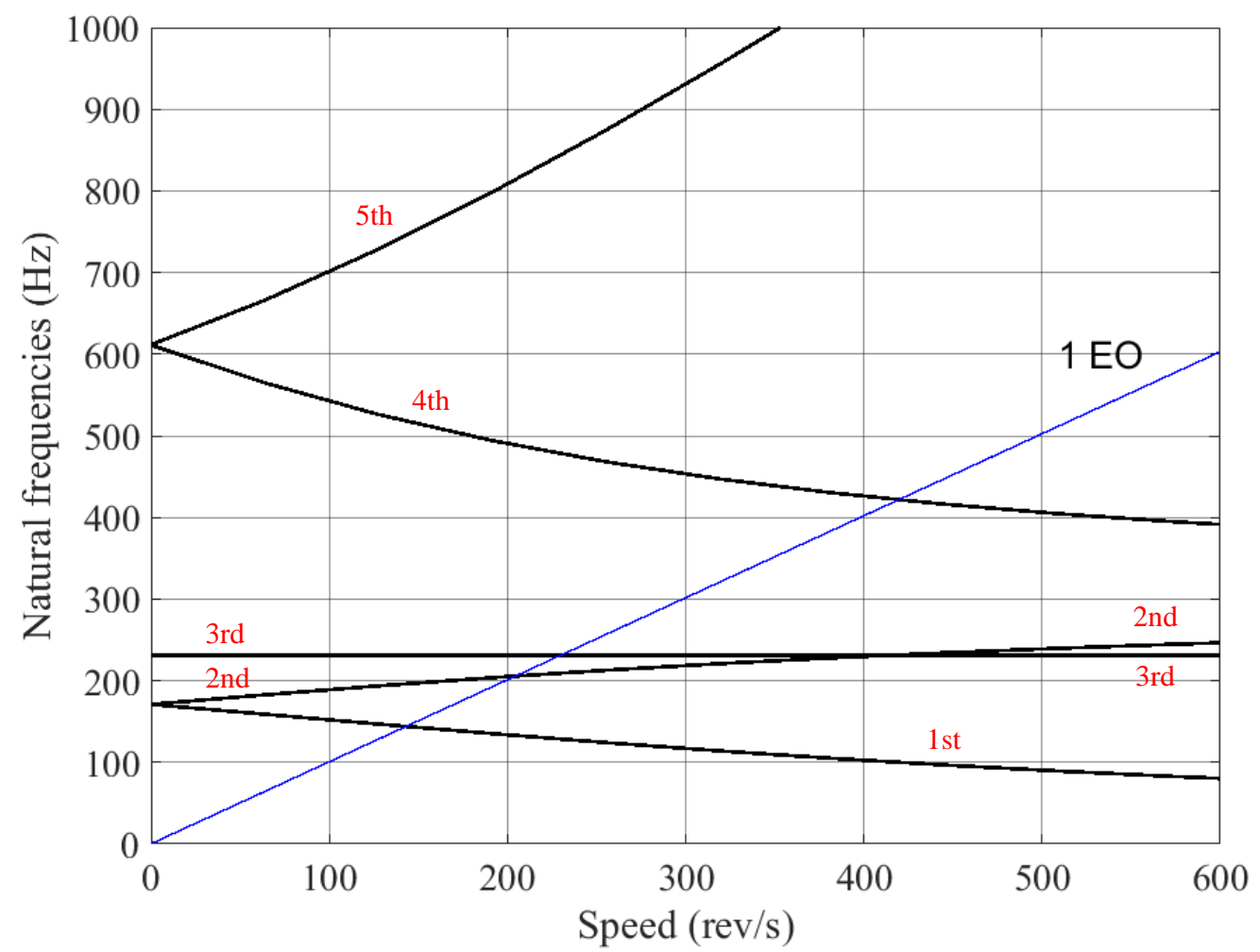

Fig. 18. Example 3a - Campbell diagram of rotating blisk supported by free-fixed shaft using ANSYS rotordynamic solver.

As expected, Fig. 18 shows that modes 1, 2, 4, and 5 are affected by gyroscopic moments due to bending of both shaft and disk. Frequency splitting was high for modes 4 and 5 due to the bending of the disk. As seen from Fig. 18, for 1 EO excitation (i.e. EO = 1, Eq. (56)), only four critical speeds exist in the selected range of speeds and these are listed in Table 6.

Table 6 Critical speeds (1 EO excitation) of blisk supported by free-fixed shaft (ANSYS rotordynamics solver).

\begin{tabular}{llllll}
\hline Mode \# & 1 & 2 & 3 & 4 & 5 \\
& & & & & \\
\hline Critical speed (rev/s) & 143.9 & 205.8 & 230.7 & 421.5 & - \\
& & & & & \\
\hline
\end{tabular}


The Campbell diagram was then generated by the new method, as per section 2.4.1, using the zero-speed modal data (Fig. 17). All the matrices in Eq. (44) were set to zero except $\mathbf{D}$ and G. The number of basis modes $H=5$. Initially, the blisk was assumed to be rigid as far as the gyroscopic effect was concerned i.e. the matrices $\mathbf{H}_{\mathbf{g}_{k}}$ were assumed identical for all points $\mathrm{P}_{k}$ on the (rigid) blisk, as explained under Eq. (27) i.e. the modal displacements $\psi_{\theta_{x_{\mathrm{P}_{k}}}}^{(r)}$, $\psi_{\theta_{y_{\mathrm{P}_{k}}}}^{(r)}$ were all equal to the values at hub (i.e. were purely due to the flexibility of the shaft). The resulting Campbell diagram (Fig. 19) correlates well with the ANSYS result only for mode nos. 1 and 2 (where the bending is mainly in the shaft, Fig. 17), and of course mode 3 (not affected by gyroscopic effect since it is torsional, Fig. 17). For modes 4 and 5 (where the zero-speed modes are dominated by flexibility of the disk, Fig. 17), Fig. 19 shows that the Campbell diagram by the new method with rigid gyroscopic effect assumption is in disagreement with the ANSYS rotordynamics solver result. This observation is also evident when comparing the critical speeds predicted by the new method (rigid disk gyroscopic effect assumption) with those from the ANSYS rotordynamics solver (Table 7), where the errors are low except for the fourth critical speed (28.7\%).

Table 7: Critical speeds (1 EO excitation) of blisk supported by free-fixed shaft (new method, rigid assumption for disc gyroscopic effect)

\begin{tabular}{llllll}
\hline Mode \# & 1 & 2 & 3 & 4 & 5 \\
\hline Critical speed (rev/s) & 146.8 & 208.5 & 230.5 & 542.5 & - \\
& & & & & \\
\hline \% discrepancy from ANSYS & 2 & 1.3 & -0.1 & 28.7 & - \\
rotordynamics solver & & & & & \\
\hline
\end{tabular}

Fig. 20 shows the result of the Campbell diagram analysis by the new method when flexibility of the disk is considered (i.e. the matrices $\mathbf{H}_{\mathbf{g}_{k}}$ were allowed to be different for 
different points $\mathrm{P}_{k}$ on the blisk). In this case, 42 inertia subdivisions (Fig. 2) were used for the blisk [See Appendix A]. It is evident that the correlation between the Campbell diagrams of the ANSYS rotordynamics solver and the proposed method (Fig. 20) for mode nos. 4 and 5 is considerably improved relative to previous result (Fig. 19). Table 8 shows that the error in $4^{\text {th }}$ critical speed becomes just $1.8 \%$. Errors in mode $1^{\text {st }}$ and $2^{\text {nd }}$ critical speeds slightly increased due to errors in the calculations of the angular modal displacements from the translational modal displacements by the vector product method of section.

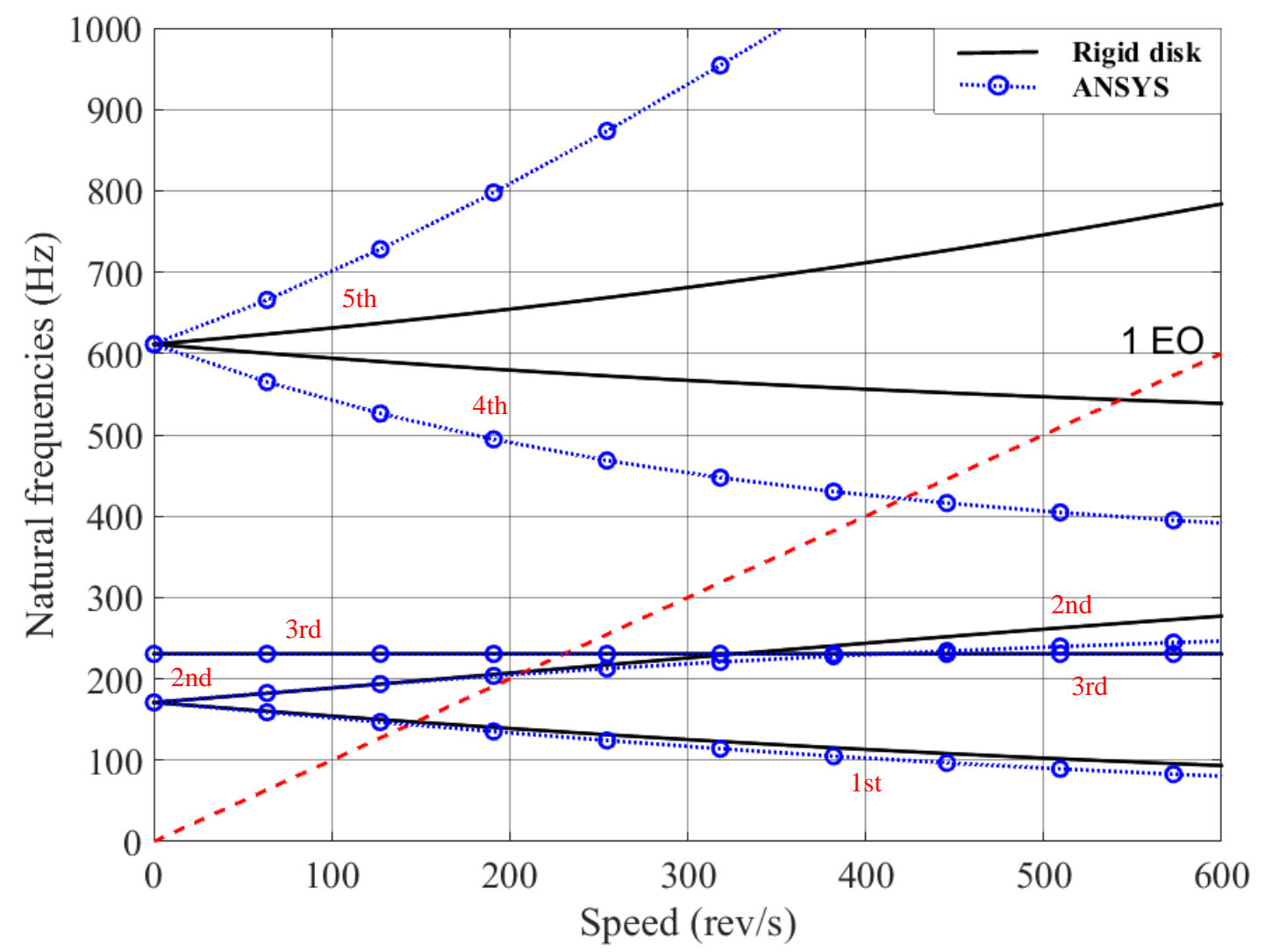

Fig. 19. Example 3a - Campbell diagram of rotating blisk supported by free-fixed shaft using the proposed method with gyroscopic effect based on rigid disc assumption.

An important advantage of the new method is the ability to produce the Campbell diagram (including gyroscopic effect with disk flexibility) in less than 1 minute with high resolution (1000 points) compared to about 2 hrs for just 11 points using ANSYS rotordynamic solver. 


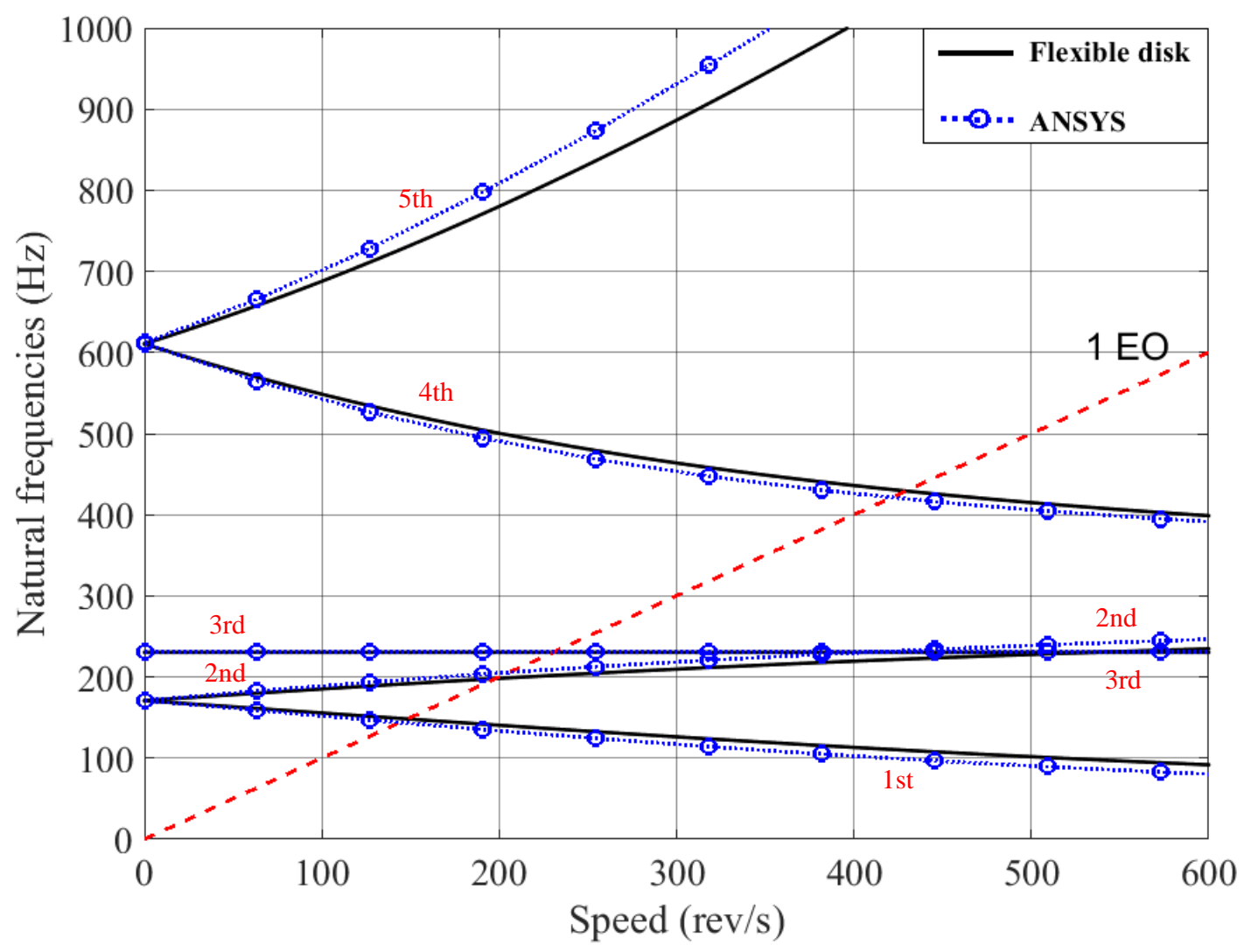

Fig. 20. Example 3a - Campbell diagram of rotating flexible blisk supported by free-fixed shaft using the proposed method with gyroscopic effect based on flexible blisk.

Table 8: Critical speeds (1 EO excitation) of flexible blisk supported by free-fixed shaft (new method, gyroscopic effect with blisk flexibility)

\begin{tabular}{llllll}
\hline Mode \# & 1 & 2 & 3 & 4 & 5 \\
\hline Critical speed (rev/s) & 148.3 & 198.1 & 230.4 & 429.3 & - \\
& & & & & \\
\hline \% discrepancy from ANSYS & 3 & -3.7 & -0.1 & 1.8 & - \\
rotordynamics solver & & & & & \\
\hline
\end{tabular}

\subsubsection{Example \# 3b-Gyroscopic moment-example from literature (Table 1)}

A model of a flexible shaft supported by 2 rigid bearings and a bladed disk of 8 blades shown in Fig. 21 was analysed by Chun and Lee in 1996 [18]. In their analysis, the supports were 
modelled as pivots (i.e. the bearing flexibility was neglected), the shaft was modelled as a beam of different cross-sections, the disk as a uniform circular plate, and the blades as uniform rectangular beams. For the case referred to as "model 1" in [18], the blades had no pre-twist and no stagger, and the results were validated against FE and experimental results published by Zhang et al. in 1994 [48]. The same case shall be analysed in this section by the method of this paper.

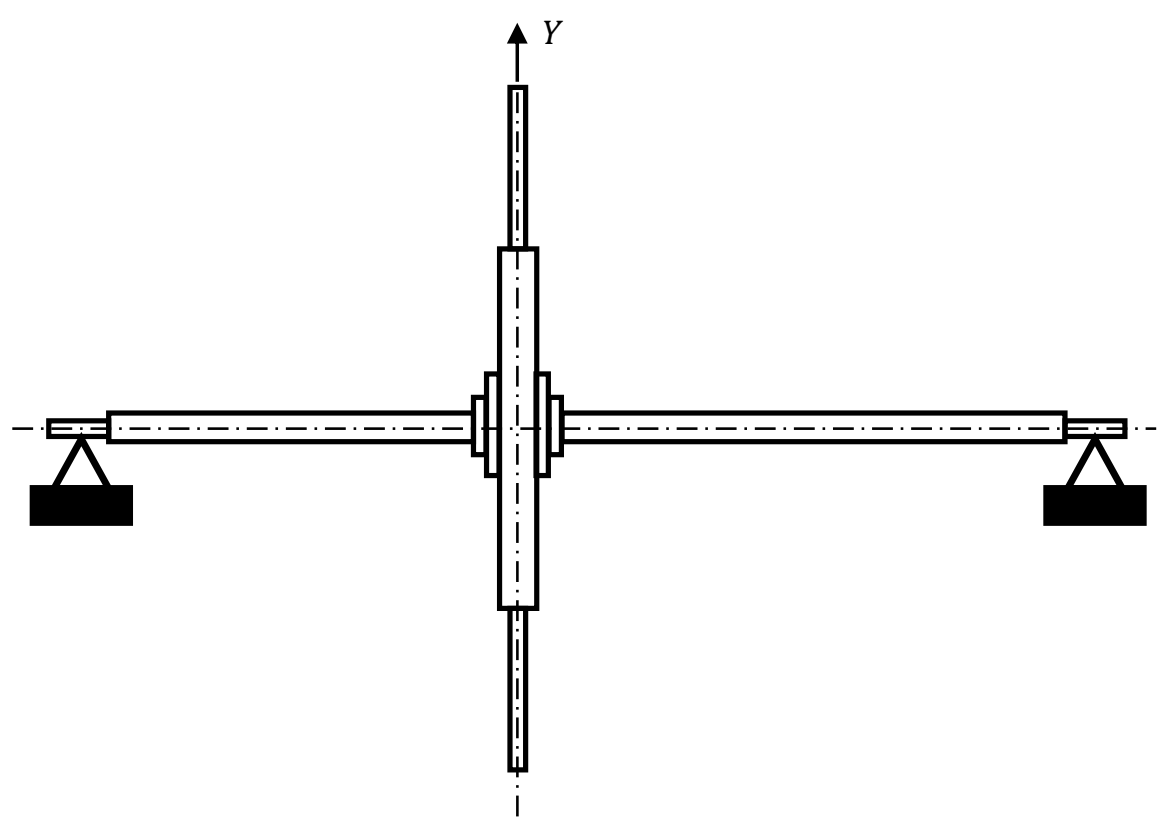

Fig. 21. Example 3b: a model of a bladed disc carried by shaft supported by 2 rigid bearings, as considered in [18].

The material properties used for the assembly are: density $7850 \mathrm{~kg} / \mathrm{m}^{3}$, Young Modulus $210 \mathrm{GPa}$, and Poisson ratio 0.28. The zero-speed modal data were generated by ANSYS using solid elements and the natural frequencies are listed in Table (9), along with the corresponding results of [18] and [48].

The Campbell diagram was generated using the present method as shown in Fig. 22. All the matrices in Eq. (44) were set to zero except $\mathbf{D}$ and $\mathbf{G}$, and the number of basis modes $H=8$. 54 inertia subdivisions (Fig. 2) were used for the blisk [See Appendix A]. The Campbell diagram generated by the present method agrees fairly well with that published in [18]. 
Table 9: Zero-speed natural frequencies

Natural frequency $(\mathrm{Hz})$

Method

\begin{tabular}{llll}
\hline 1 & 2 & 3 & 4
\end{tabular}

\begin{tabular}{lllll}
\hline FE [present work] & 46.8 & 46.8 & 61.3 & 61.3
\end{tabular}

\begin{tabular}{llllc}
\hline Analytical [18] & 47.17 & 47.17 & 59.92 & 59.92 \\
& & & & \\
\hline FE [48] & 46.0 & 46.0 & 60.15 & 60.15 \\
& & & & \\
\hline Experimental [48] & 48.0 & 48.0 & - & -
\end{tabular}

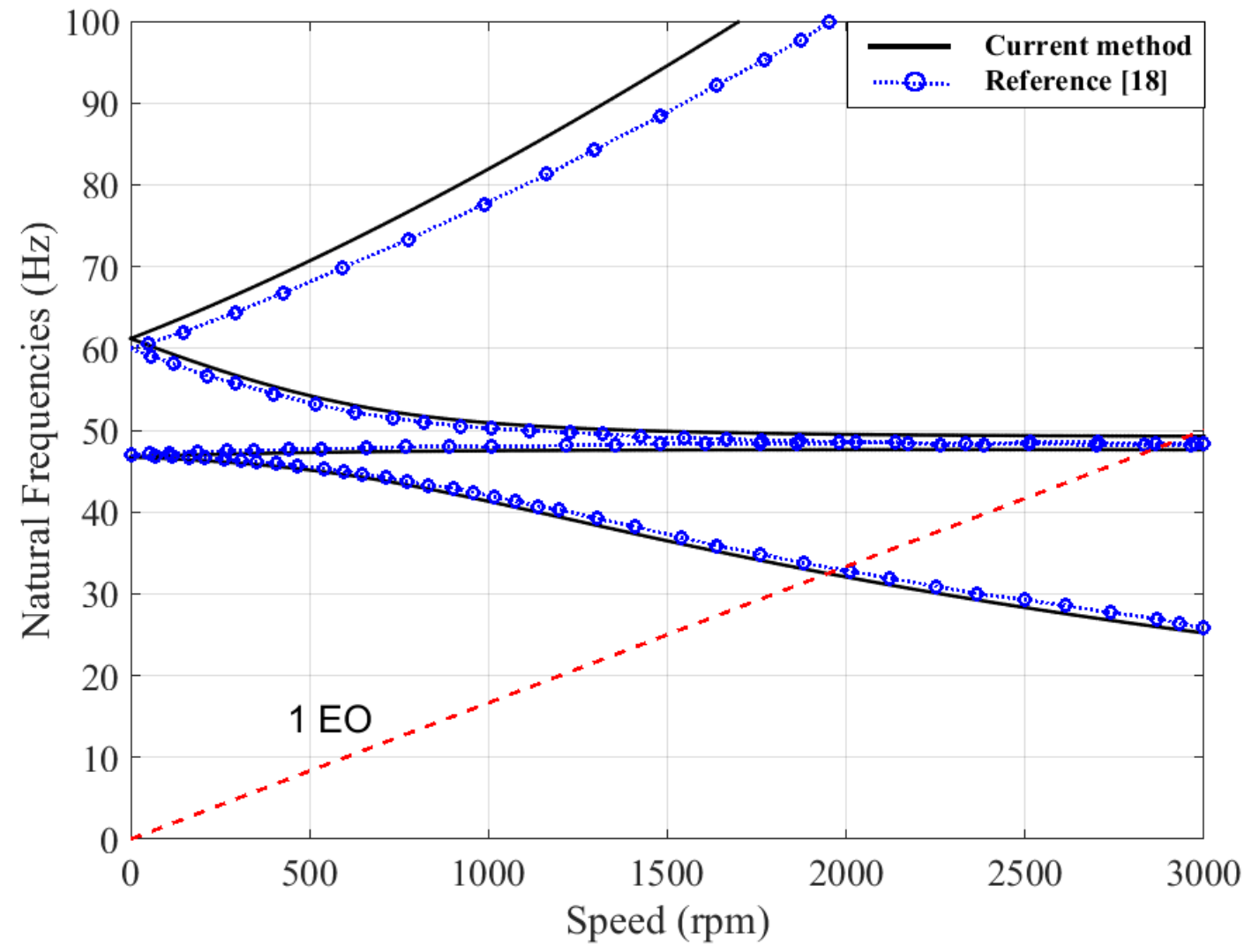

Fig. 22. Example 3b - Campbell diagram of system in Figure 21 using proposed method. 
A travelling wave excitation was then applied to the blade tips under a steadily increasing speed condition and at fixed engine order (EO). In this type of excitation, the force on the tip of blade no. $n$ is given by [51]:

$f_{n}(t)=A \sin \left[\operatorname{EO}\left(\Omega_{\mathrm{i}} t+\left(\frac{\Omega_{\mathrm{f}}-\Omega_{\mathrm{i}}}{2 T_{\mathrm{s}}}\right) t^{2}\right)+\frac{2 \pi n N_{d}}{N_{\mathrm{b}}}\right]$

where $N_{\mathrm{b}}$ is the total number of blades, and $N_{d}$ is the number of nodal diameters. Fig. 23 shows the simulator response (as per section 2.4.2) at one of the blade tips in the $x$-direction for $A=1$ and EO $=1$ and $T_{\mathrm{s}}=2 \mathrm{sec}, \zeta_{r}=0.05$ (Eq. (5)). The figure shows that the resonances due to the excitation of the first, second, and third modes agree with the first three critical speeds (for 1EO excitation) indicated in Fig. 22.

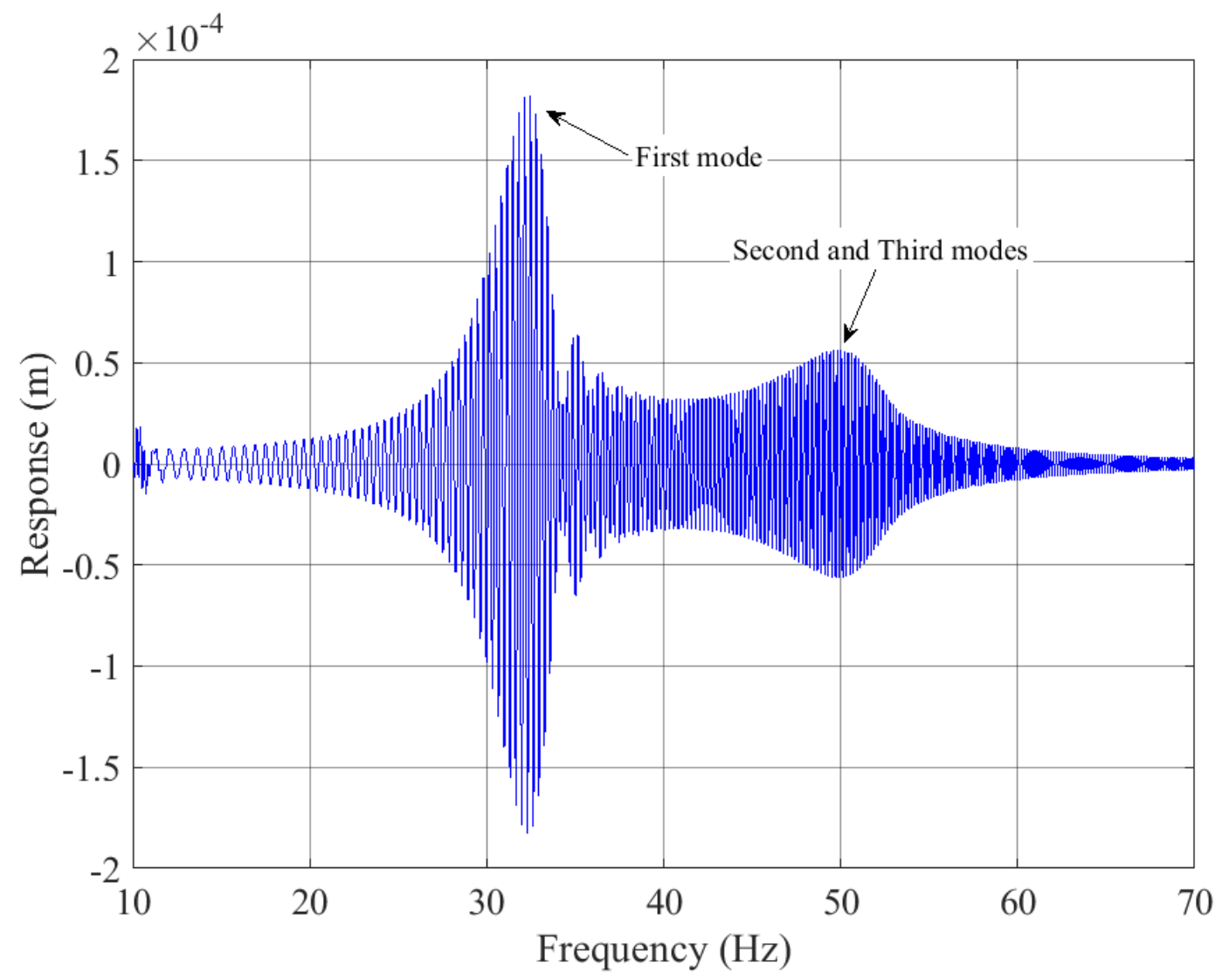

Fig. 23. Example $3 b$ - Forced response of system in Figure 20 using proposed method. 


\subsection{Example \# 4 - Gyroscopic, Coriolis, Spin Softening, Centrifugal Stiffening}

The system shown in Fig. 24 was first solved for the modal data by Ma et al. [2] in 2015. They examined the effects of rotation analytically, numerically (FE), and experimentally. Not all natural frequencies were validated experimentally, so only those validated by experiments will be considered here. In their analytical analysis the authors in [2] used Hamilton's principle in conjunction with the assumed modes method.

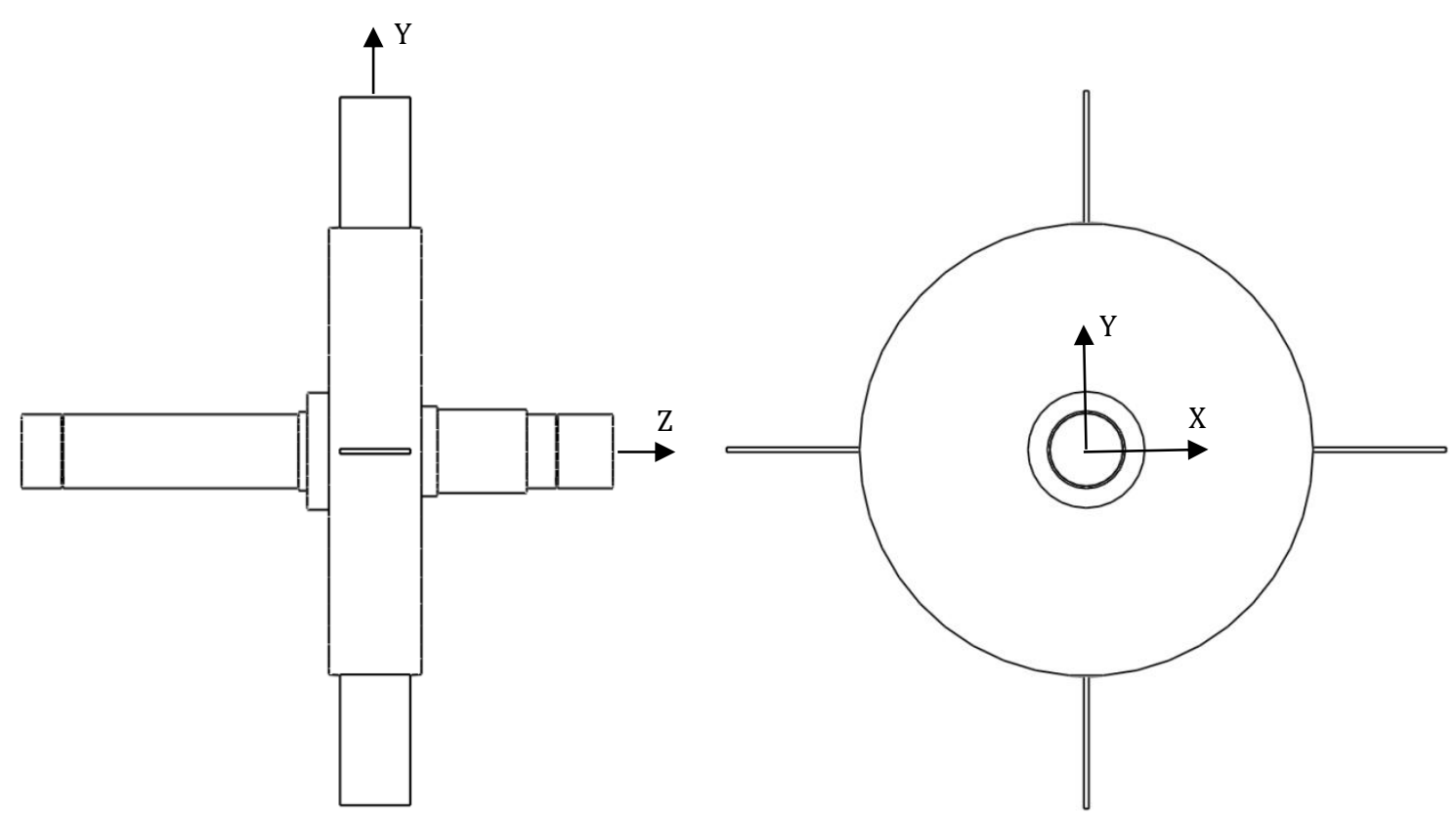

Fig. 24. Example 4 - rotating assembly considered in [24]

The system consists of a stepped circular cross-section shaft supported by two bearings (with equal stiffness in the vertical and horizontal direction $1.5 \times 10^{7} \mathrm{~N} / \mathrm{m}$ ) carrying a disk with four blades, the material is the same for all components (density $7800 \mathrm{~kg} / \mathrm{m}^{3}$, Young Modulus $200 \mathrm{GPa}$ ). SolidWorks and ANSYS used to create the FE model for the zero-speed data. The mesh was refined on all blades and the outer surface of disk in order to improve the results, and the total number of degrees of freedom was 1800858 . The boundary conditions were as follows: both ends of the shaft were constrained from axial motion; left hand end constrained from torsional motion. Fig. 25 shows the mode shapes of the system at zero 
speed, as obtained by ANSYS. The zero-speed natural frequencies are presented in Table 10 where they are also compared with the results published in [2].
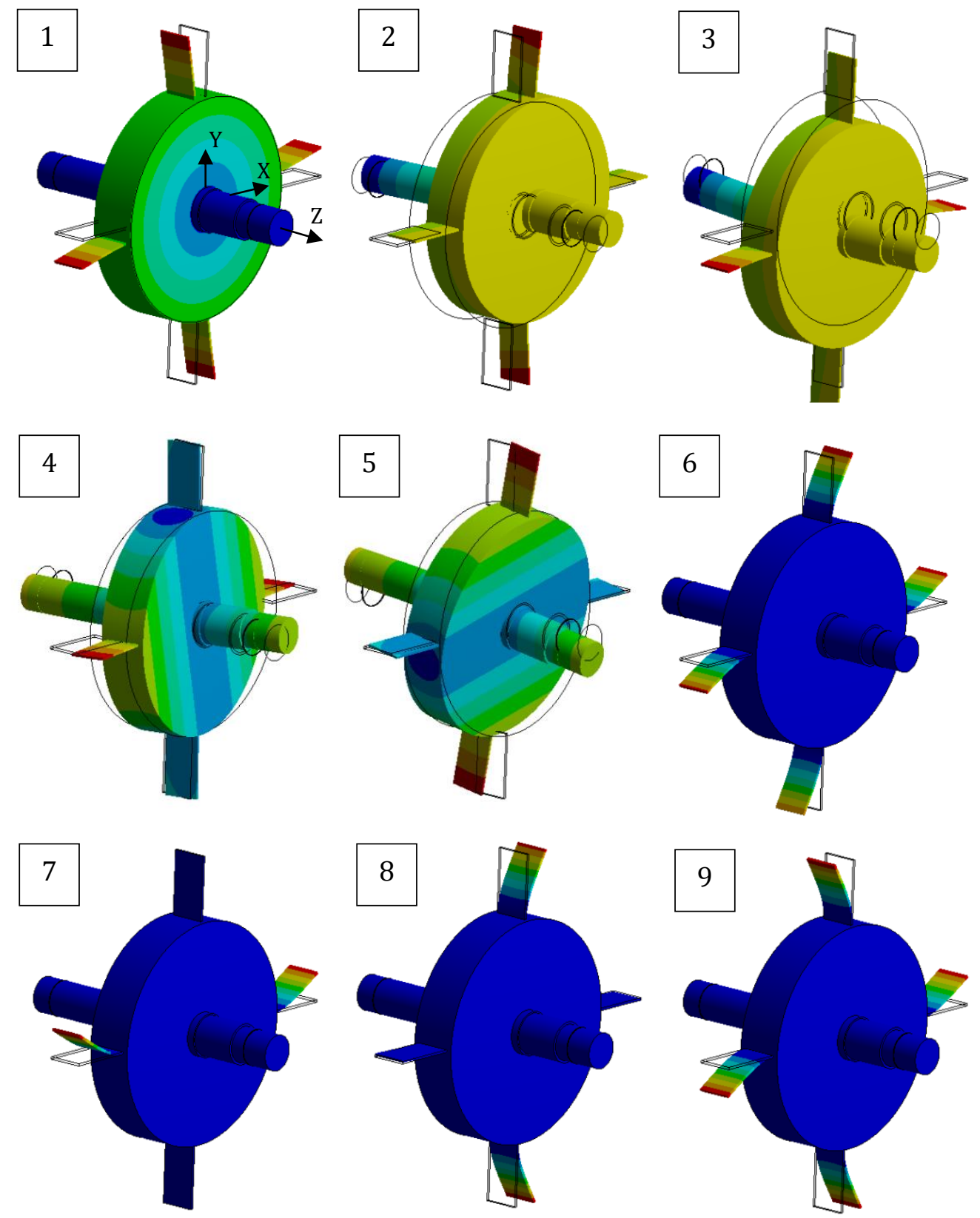

Fig. 25. Example 4 - mode shapes of the non-rotating assembly. 
Table 10: Natural frequencies of the non-rotating assembly.

\begin{tabular}{lccccccccc}
\hline Mode & 1 & 2 & 3 & 4 & 5 & 6 & 7 & 8 & 9 \\
& & & & & & & & & \\
\hline FE [present] (Hz) & 121.7 & 133.8 & 133.8 & 266.5 & 266.5 & 364.3 & 365 & 365 & 370.4 \\
& & & & & & & & & \\
\hline FE [2] (Hz) & 117.4 & 132.9 & 132.9 & 269.6 & 269.6 & 364.7 & 365.4 & 365.4 & 370.7 \\
& & & & & & & & & \\
\hline Experimental [2] (Hz) & - & 129.3 & 129.3 & 258 & 258 & 361.3 & 364.7 & 368 & 372 \\
& & & & & & & & & \\
\hline
\end{tabular}

The zero-speed modal data were then used in the proposed method in order to generate Campbell diagram as per section 2.4.1. The number of basis modes $H=9$. The number of inertia divisions is 42 [See Appendix A]. First, only the gyroscopic effects were considered, as shown in Fig. 26. It is clear that the affected modes were those featuring some inclination of the disk (Fig. 25): the $2^{\text {nd }}, 3^{\text {rd }}$ modes (slightly affected) and the $4^{\text {th }}, 5^{\text {th }}$ modes (highly affected). This agrees with what was reported in [2].

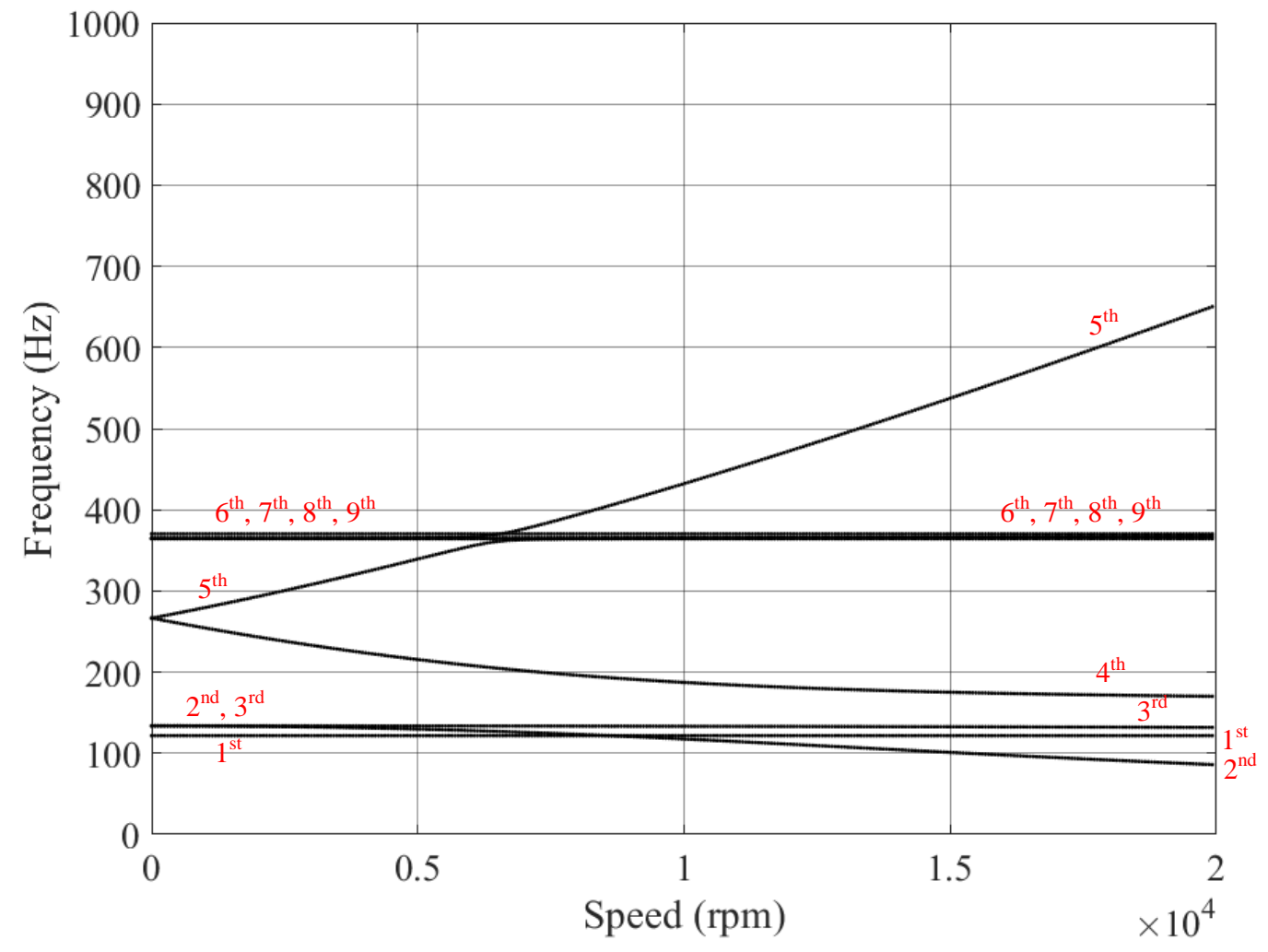

Fig. 26. Example 4 - Campbell diagram with gyroscopic effect only. 


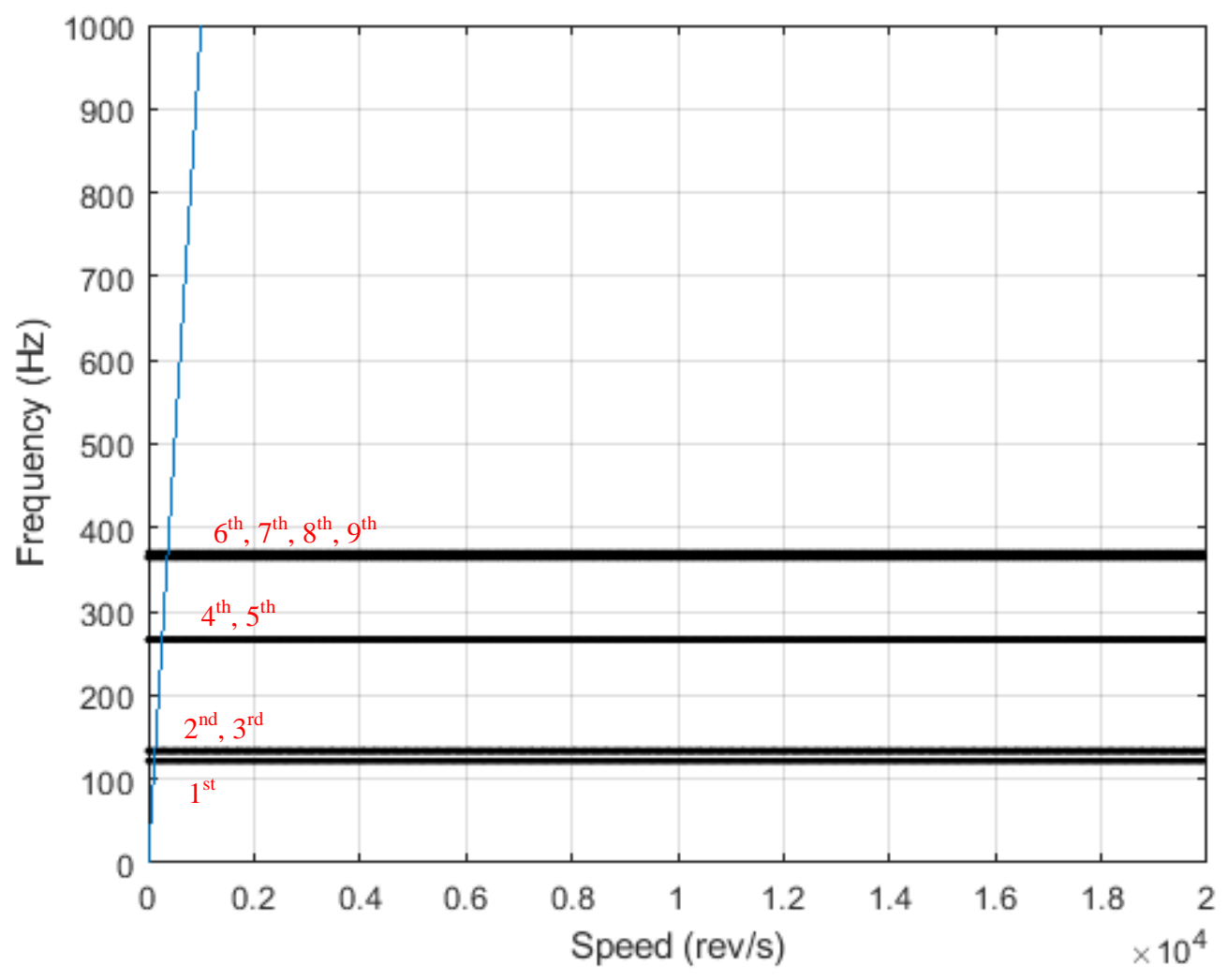

Fig. 27. Example 4 - Campbell diagram with Coriolis effect only.

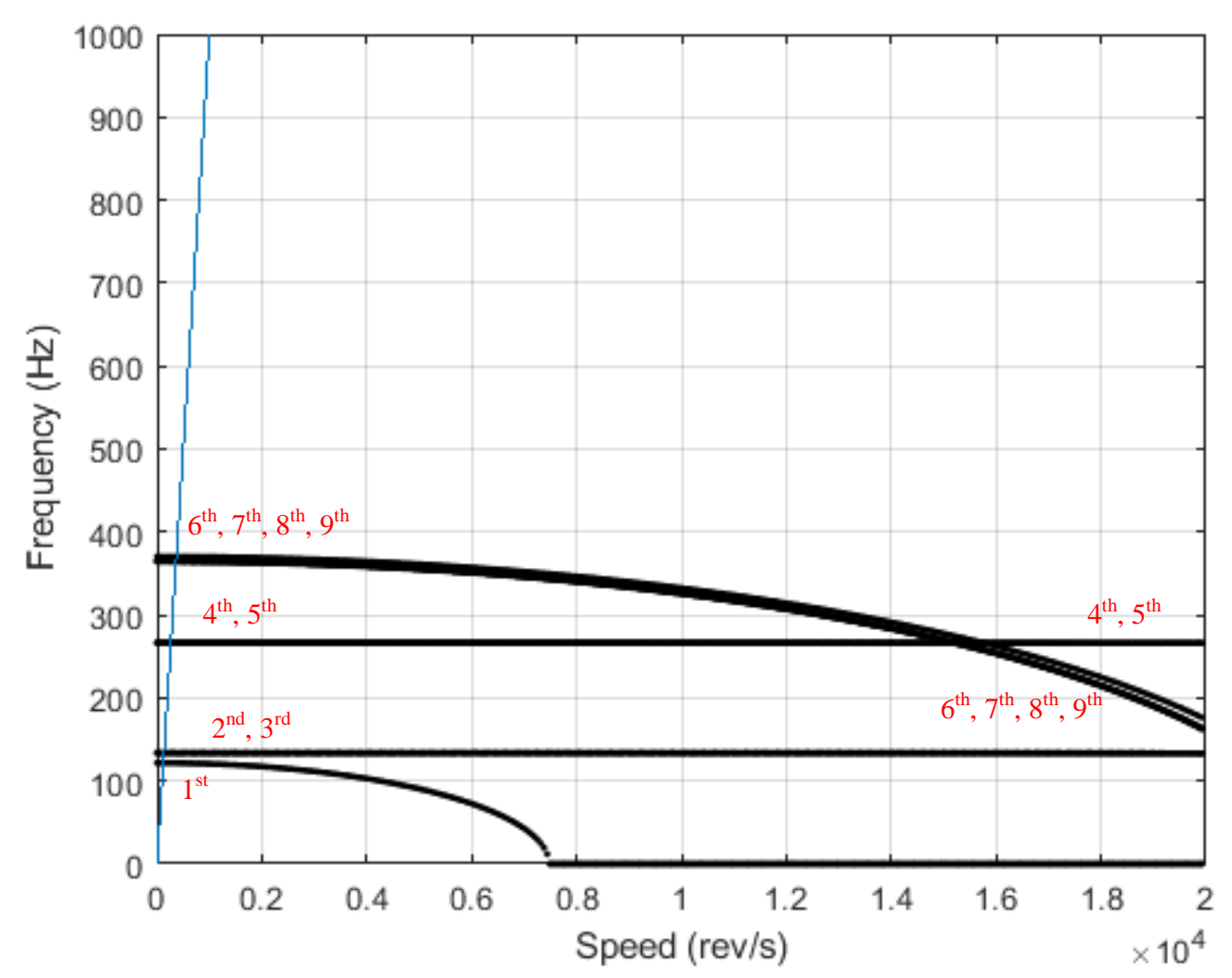

Fig. 28. Example 4 - Campbell diagram with spin with softening effect only. 


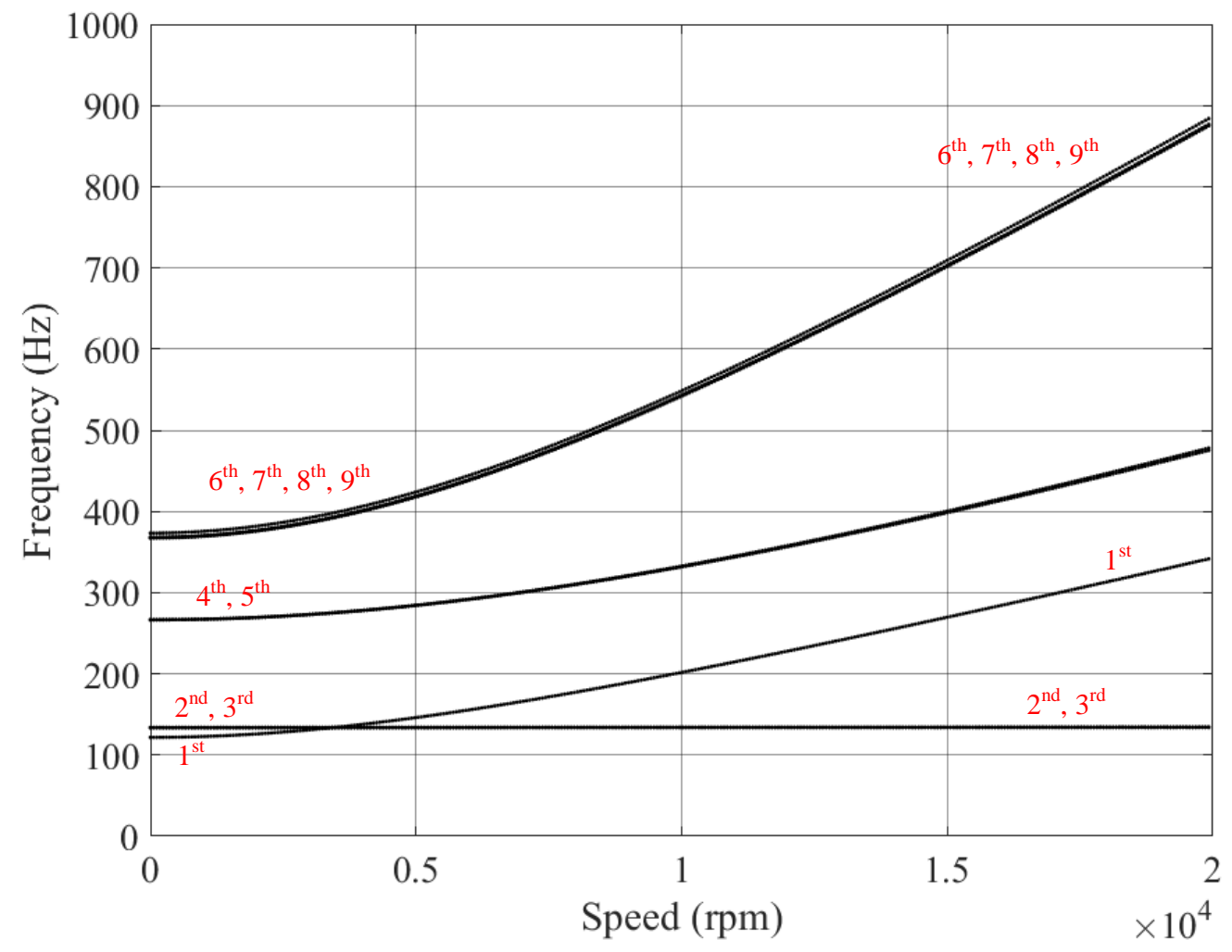

Fig. 29. Example 4 - Campbell diagram with centrifugal stiffening effect only.

Secondly, the effects of Coriolis only (Fig. 27) and spin-softening only (Fig. 28) were examined. It is clear that in case of Coriolis, no modes are affected, which justifies the assumption made in [2] of neglecting Coriolis. In case of spin-softening, mode nos. 2-5 are unaffected, which justifies another assumption made in [2] that spin-softening effects came only from the blades (the affected modes 1, 6-9 involve little or no bending vibration in either shaft or disc, as per zero-speed modes in Figure 25).

The $3^{\text {rd }}$ trial included the centrifugal stiffening effects, where the effects of the individual components $\left(M_{x}, M_{y}, M_{z}\right)$ of the centrifugal stiffening moment $\mathbf{m}_{k}$ (Eq. (20) were examined. By examining the zero-speed mode shapes in Fig. 25, it is clear that mode no. 6 is the only mode affected by $M_{x}$, and mode no. 4 is the only mode affected by $M_{y}$. The modes affected by $M_{z}$ are mode nos. 1, 6-9. Hence, considering all three components of $\mathbf{m}_{k}$, all modes would be affected except for the $2^{\text {nd }}$ and $3^{\text {rd }}$, as shown in Fig. 29. 
The last step was to consider all effects at the same time and comparing them to theoretical results published in [2]. The same assumptions made in [2] were applied to the rotational inertia matrices (Eqs. (38-41)). These have been shown to be consistent with the previous results in Figures 26-29 and are summarised as follows:

- Gyroscopic effects were assumed to come only from shaft and disk, i.e. the gyroscopic effect from the blade inertia was ignored in [2].

- The disk was assumed to be rigid in [2].

- Centrifugal stiffening and spin-softening effects were assumed to come only from the blades in [2].

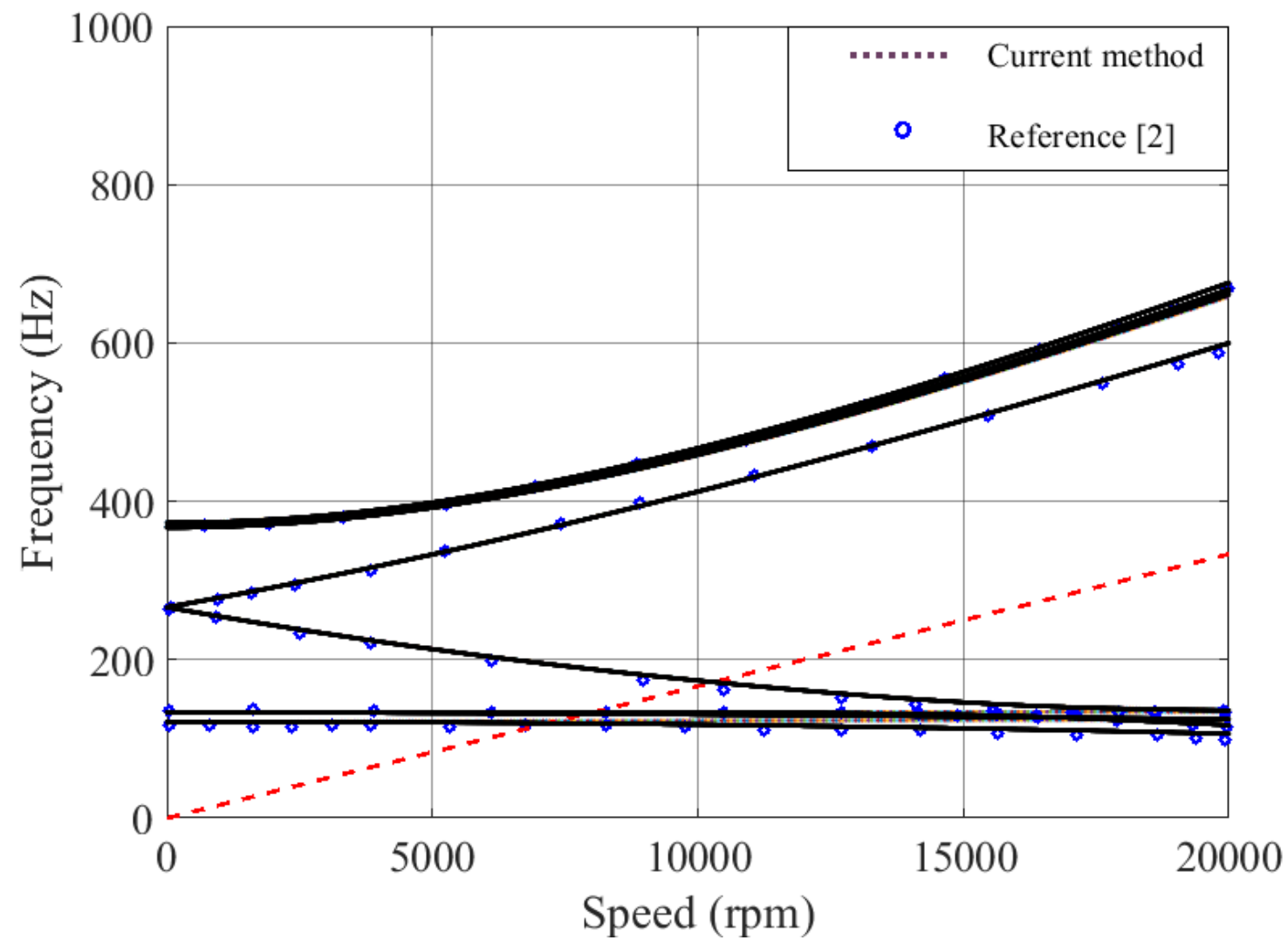

Fig. 30. Example 4 - Campbell diagram with all rotational effects included.

The resulting Campbell diagram in Fig. 30 is found to be in a good agreement with that published in [2]. 


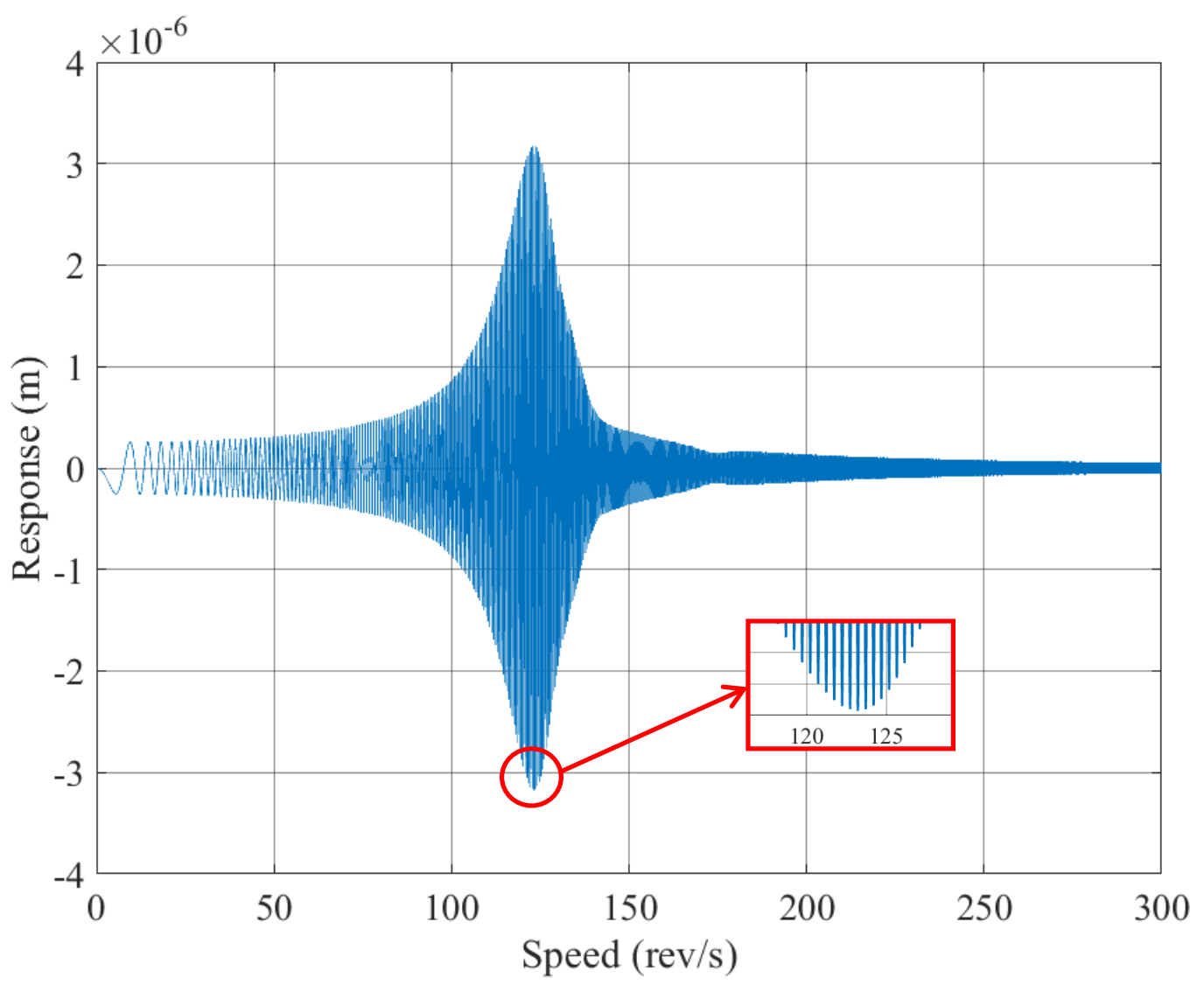

Fig. 31. Forced response (first critical speed)

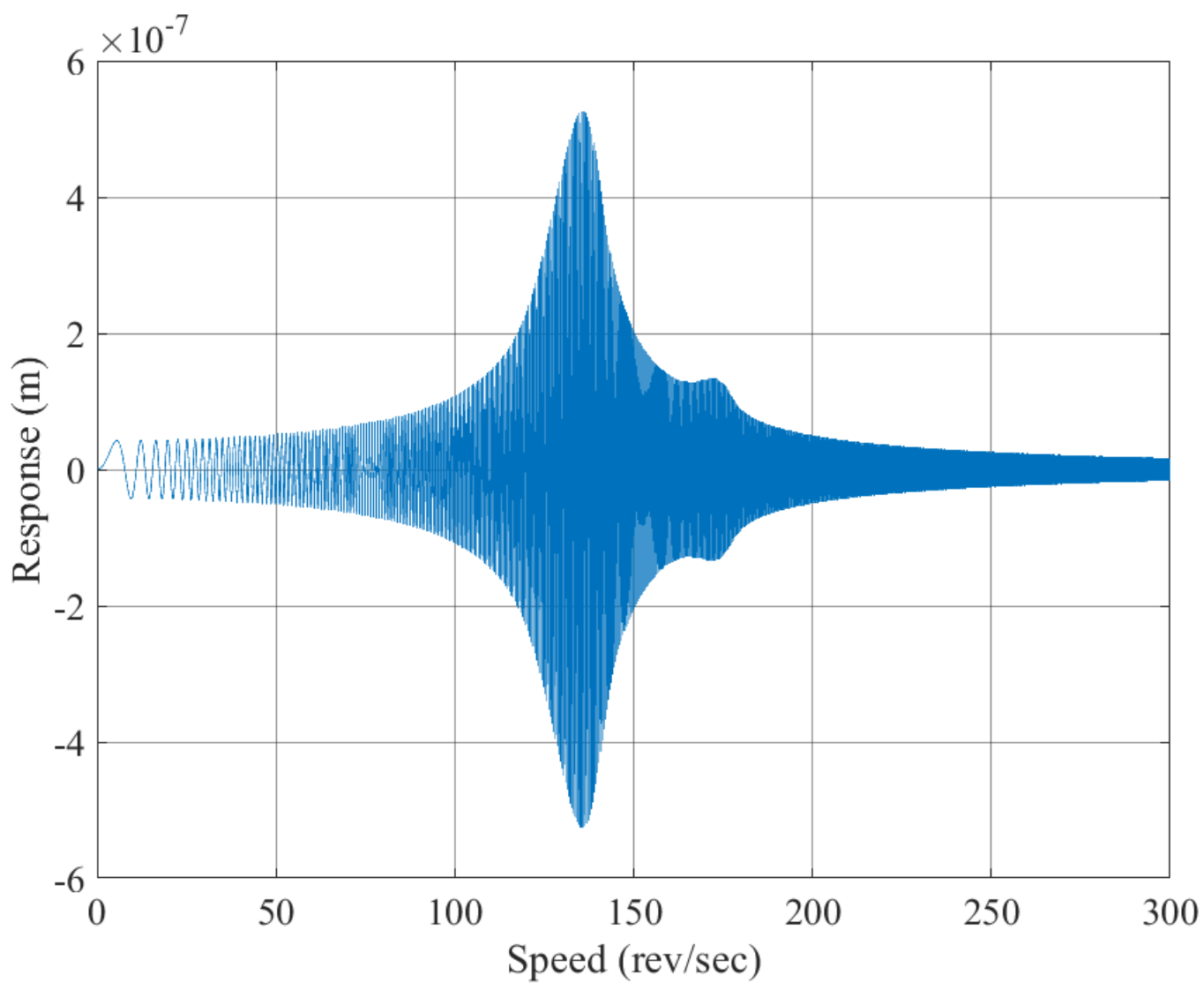

Fig. 32. Forced response (second and third critical speeds) 
The Campbell diagram in Fig. 30 was generated in just 1 or 2 minutes, depending on how many speed steps were required. To do the same analysis with ANSYS rotordynamics solver, the PRESTRESS command should be used for centrifugal stiffening and spin softening, and CORIOLIS command should be activated in modal analysis for the gyroscopic effect. Such an analysis was found to take a very long time, since it required, for each speed value, a separate analysis to generate the solution. This further validates the new method proposed in this paper.

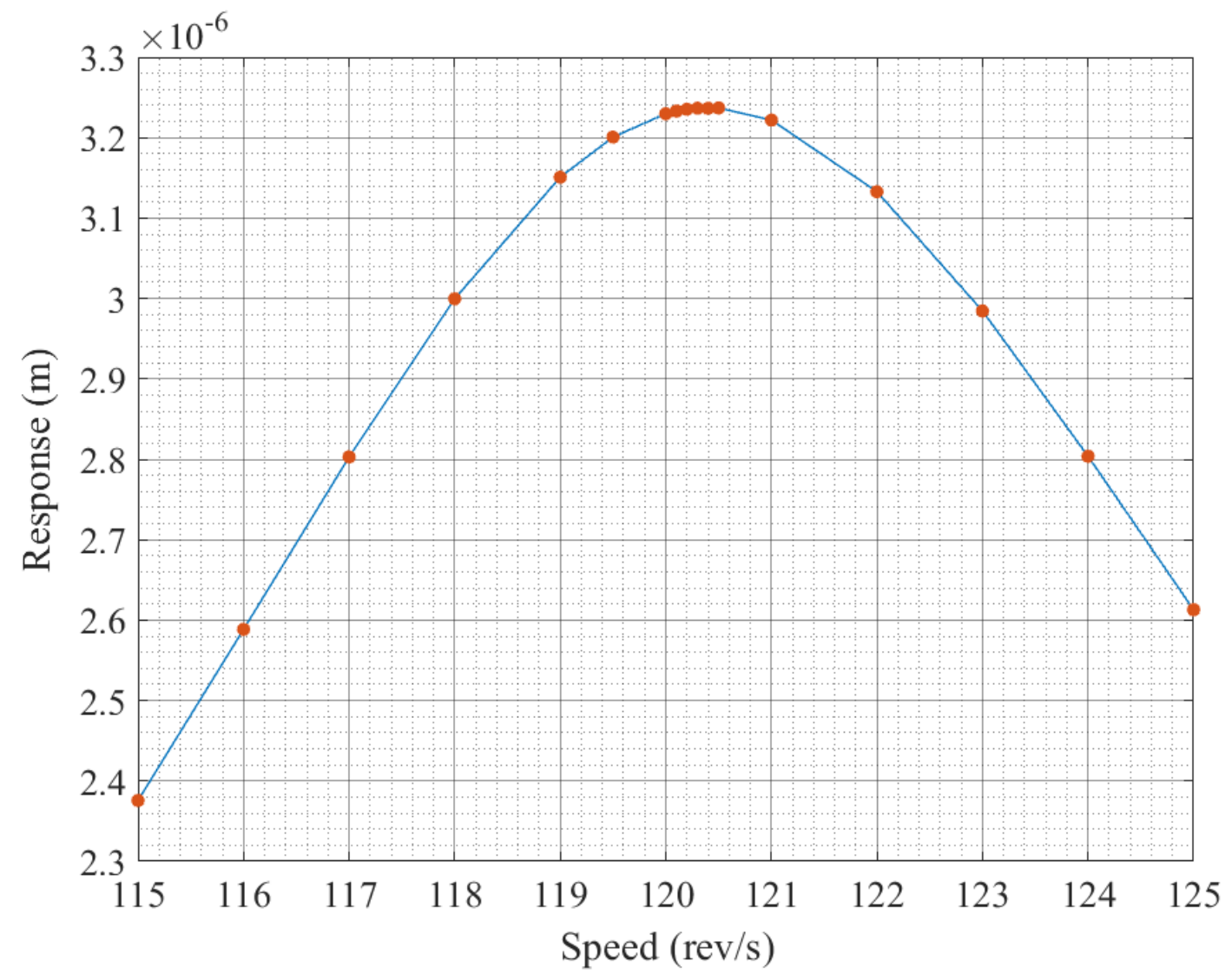

Fig. 33. Steady state Forced response around the first critical speed

The critical speeds with respect to a $1 \mathrm{EO}$ excitation (i.e. EO =1, Eq. (56)), are identified in Fig. 30 and presented in Table 11. 
Table 11: Critical speeds (1 EO excitation) for Campbell diagram of Fig. 30

\begin{tabular}{lccc}
\hline Mode \# & 1 & 2 & 3 \\
& & & \\
\hline Critical speed (rev/sec) & 120.15 & 133.5 & 171.7
\end{tabular}

Critical speed (rev/sec)

$120.15-133.5$

Fig. 31 and Fig. 32 show the forced responses at two different blades tips in x-direction due to a chirp excitation at one of the blades tips nodes (normal to the blade) under variable speed condition, as per Eq. (56) with $A=1$ and $\mathrm{EO}=1$ and $T_{\mathrm{s}}=5 \mathrm{sec}, \zeta_{r}=0.05$ (Eq. (5)). The resonance frequencies agree with the critical speeds listed in Table 11. However, there are slight errors in their values, since the response is transient and not strictly steady-state. In order to confirm that, the simulation was repeated at a number of fixed speed values $\Omega_{k}$ around the first critical speed (i.e. in Eq. (56), $\Omega_{\mathrm{i}}=\Omega_{\mathrm{f}}=\Omega_{k}$ ) so that the system reaches steady-state for each $\Omega_{k}$. The results are plotted in Fig. 33 for first critical speed, confirming the agreement between the forced response and the Campbell diagram.

\section{CONCLUSIONS}

This paper has introduced a novel methodology to include rotational effects (centrifugal stiffening, Coriolis forces, spin softening and gyroscopic moments) in a generic shaft-blisk system, using zero-speed modal data. The method does not require additional FE analysis at each speed or the derivation of equations from first principles. This research was motivated by the need to upgrade a shaft-blisk simulator designed to generate blade tip timing (BTT) data for the development and validation of BTT algorithms. To ensure computational efficiency, the dynamic response of the existing simulator was governed by modal equations based on the eigenvectors and eigenvalues of the FE analysis at zero-rotational speed. The upgraded simulator equations remained based on the same speed-independent eigenvectors 
since the rotation-induced inertia effects were introduced as "external" excitations at discrete locations. The methodology was progressively validated using six examples, its accuracy checked against results from the literature and commercial software. A notable observation is the high computational speed in generating a Campbell diagram for gyroscopic effects on a flexible disk, or for centrifugal effects, where the new method was found to deliver the diagram with high resolution (1000 points) over a wide speed range ( $0 \mathrm{rpm}$ to $60000 \mathrm{rpm})$ in a few minutes, compared to a few hours taken by commercial FE rotordynamics software. Such computational speed is essential for the generation of BTT data by the simulator under forced conditions, since the process of determining the blade passing time (past a given probe) involves multiple calculations in the integration process for the forced response (including revisions in the time step) [42]. Future research will focus on the inclusion of the parametric excitation term (Eq. (55)) arising from the shaft vibration.

\section{ACKNOWLEDGMENT}

We would like to thank the Egyptian Ministry of Higher Education, and the British Council in Cairo for funding this project.

\section{APPENDIX A}

With reference to Fig. 2, the disc is divided into $N_{r}$ radial segments of constant thickness, each radial segment is divided into $N_{a}$ angular segments, and each blade is divided into $N_{b r}$ radial segments.

\section{Polar moment of inertia}

For the blade segment shown in Fig. A.1: 


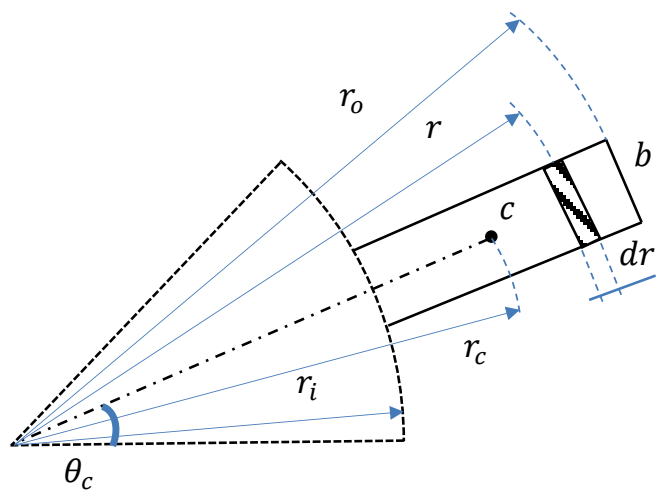

Fig. A.1. Blade division element

$$
\Delta I=\int_{r_{i}}^{r_{0}} r^{2} \rho t b d r=\rho t b\left(\frac{r^{3}}{3}\right)_{r_{i}}^{r_{o}}=\frac{\rho t b}{3}\left(r_{o}^{3}-r_{i}^{3}\right)
$$

where $t, \rho$ are the thickness and density respectively.

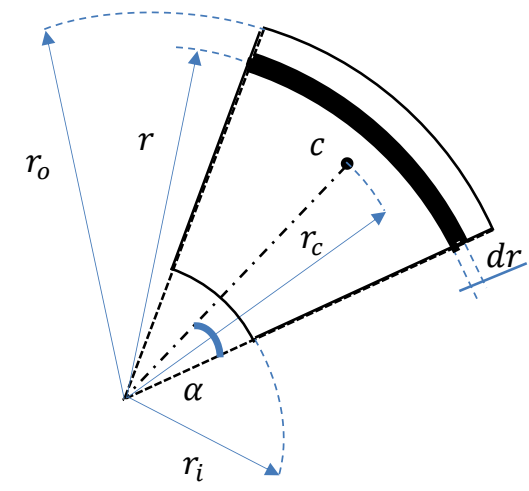

Fig. A.2. Disk division

For the disc segment shown in Fig. A.2.

$$
\Delta I=\int_{r_{i}}^{r_{o}} r^{2} \rho t(2 r \alpha) \mathrm{d} r=2 \rho t \alpha \int_{r_{i}}^{r_{o}} r^{3} \mathrm{~d} r=\frac{\rho t \alpha}{2}\left(r_{o}^{4}-r_{i}^{4}\right)
$$




\section{Centre of mass}

The position of the centre of mass of each segment (denoted by $\mathrm{P}_{k}$ in the main text) is determined by two components (angular and radial). In the case of a blade segment no. $i$ :

$\theta_{c_{i}}=\theta_{c_{1}}+2 \alpha_{\mathrm{b}}\left(n_{i}-1\right) \quad, \quad n_{i}=1,2, \ldots \ldots, N_{\mathrm{b}}$

where $\theta_{c_{i}}$ is the angle of the segment no. $i$ centre measured from the positive $x$-axis, $\theta_{c_{1}}$ is the angle of first blade, $\alpha_{\mathrm{b}}$ is the angular difference between consecutive blades assuming that the first blade is in line with the positive $x$-axis, and $N_{\mathrm{b}}$ is the total number of blades. The radial position of segment centre is

$r_{c_{i}}=\frac{r_{i}+r_{0}}{2}$

Where $r_{i}$ and $r_{o}$ are the inner and outer radii of the segment.

a)

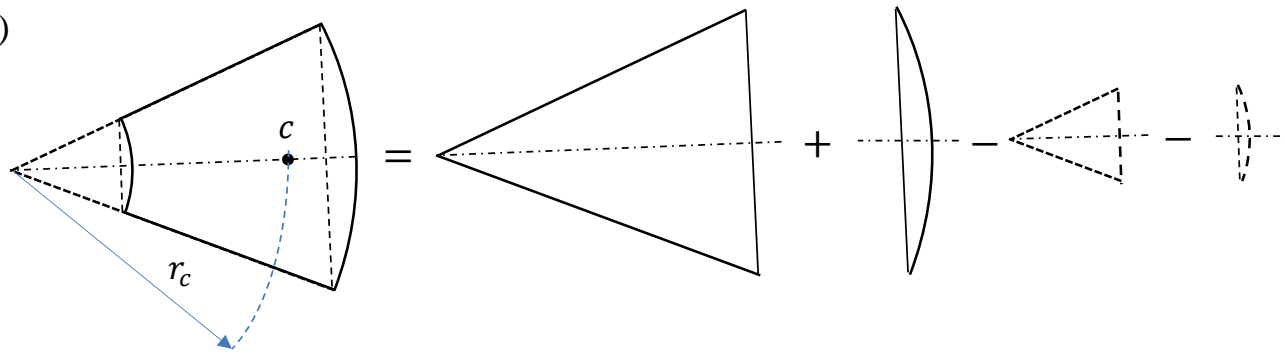

b)

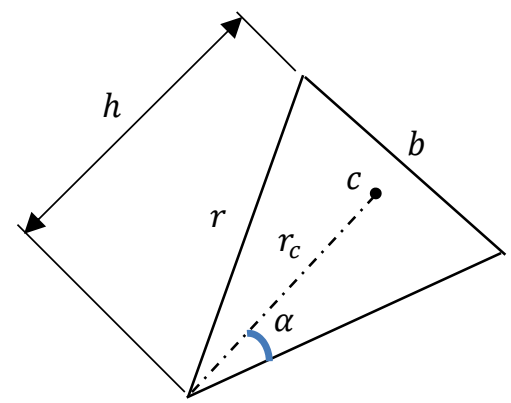

c)

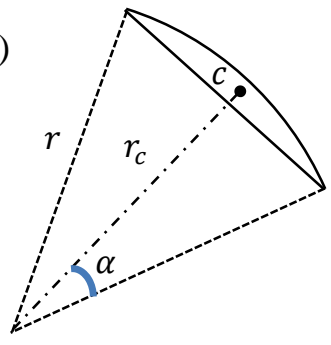

Fig. A.3. Centre of mass of disk divisions 
In the case of a disc segment no. $i$ :

$\theta_{c_{i}}=\theta_{c_{1}}+2 \alpha_{d}\left(n_{i}-1\right) \quad, \quad n_{i}=1,2, \ldots \ldots, N_{a}$

where $\alpha_{d}$ is the angular difference between every two angular segments of the disc, and $N_{a}$ is the total number of angular divisions. In order to obtain $r_{c_{i}}$, the segment is divided into 4 parts, as shown in Fig. A.3(a).

For segments 1 and 3 shown in Fig. A.3(b)

$h=r \cos \alpha, \quad b=2 r \sin \alpha \quad \alpha$ is half the segment angle

$A=b r \cos (\alpha) / 2=r^{2} \sin \alpha \cos \alpha$

$r_{c}=\frac{2}{3} r$

For segments 2 and 4 shown in Fig. A.3(c)

$$
\begin{aligned}
& A=\frac{1}{2} r^{2}(2 \alpha-\sin 2 \alpha) \\
& r_{C}=\frac{4 r(\sin \alpha)^{3}}{3(2 \alpha-\sin (2 \alpha))}
\end{aligned}
$$

Finally, the radius of the segment's centre of mass is calculated as

$$
r_{c_{i}}=\frac{A_{1} r_{c_{1}}+A_{2} r_{c_{2}}-A_{3} r_{c_{3}}+A_{4} r_{c_{4}}}{A_{1}+A_{2}-A_{3}+A_{4}}
$$




\section{APPENDIX B (Table of Nomenclature)}

$\mathrm{A}_{1} \quad$ Coriolis effect matrix

$\mathrm{A}_{2} \quad$ Spin softening matrix

$\mathrm{A}_{3} \quad$ Angular acceleration matrix

C Modal damping matrix

D Diagonal matrix containing the squares of the natural frequencies

EI The flexural rigidity of a beam

EO Engine Order

$F_{\mathrm{Z}_{\mathrm{P}}}(t) \quad$ A harmonic force

$\mathrm{f}(t) \quad$ Vector of external dynamic excitation forces

G Gyroscopic effect matrix

$\mathrm{g}_{k} \quad 2 \times 1$ moment vector containing the gyroscopic effect

$H \quad$ Number of modes used in transformation

$\mathrm{H}_{\mathrm{g}_{k}}, \mathrm{H}_{\mathrm{h}_{k}}$, Zero-speed modal matrices whose $H$ columns are the mass-normalised $\mathrm{H}_{\mathrm{m}_{k}} \quad$ eigenvectors evaluated at the degrees of freedom associated with $\mathrm{g}_{k}, \mathrm{~h}_{k}$, and $\mathrm{m}_{k}$ respectively

$\mathrm{H}_{\mathrm{P}}, \mathrm{H}_{\mathrm{f}} \quad$ Zero-speed modal matrices.

$\mathrm{h}_{k} \quad 2 \times 1$ force vector of the Coriolis and spin-softening effects

$\mathrm{h}_{0_{k}} \quad 2 \times 1$ vibration-independent rotational force vector

$\mathrm{i}, \mathrm{j}, \mathrm{k} \quad$ Unit vectors parallel to axes $X, Y, Z$ (Fig.1)

$\mathrm{i}^{\prime}, \mathrm{j}^{\prime} \quad$ Unit vectors parallel to $U, V$ (Fig. 1)

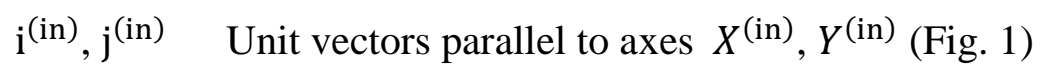

$L \quad$ Beam length

$\mathrm{L}_{k} \quad$ Angular momentum of a segment with mass centre $\mathrm{P}_{k}$

$M_{Z \mathrm{P}_{k}}$, Induced moments in the edge-wise $(U V)$ and flap-wise (UZ) planes due to the $M_{v_{\mathrm{P}_{k}}} \quad$ centrifugal tension $N_{\mathrm{P}_{k}}$

$m \quad$ Mass per unit length

$\mathrm{m}_{k} \quad$ A $3 \times 1$ moment vector of the centrifugal stiffening effect

$\mathrm{N} \quad$ Centrifugal stiffening matrix

$N_{\mathrm{P}_{k}} \quad$ The centrifugal tension on a segment with mass centre $\mathrm{P}_{k}$ of radial extent $\Delta R_{\mathrm{P}_{k}}$

$\mathrm{n}_{\text {def }_{k}}(t) \quad$ The variable part of $\mathrm{n}_{k}$ due to its change in direction 


\begin{tabular}{|c|c|}
\hline $\mathrm{n}_{k}$ & A unit vector originally parallel to $\mathrm{k}$ \\
\hline$Q_{\mathrm{cr}}$ & The critical buckling load of the cantilever \\
\hline $\mathrm{q}(t)$ & The $H \times 1$ vector of modal coordinates \\
\hline$R, R_{\mathrm{P}_{k}}$ & radial coordinate of $\mathrm{P}, \mathrm{P}_{k}$ in rotating frame $X Y($ Fig. 1,2$)$ \\
\hline $\mathrm{r}$ & The position vector of an arbitrary point $\mathrm{P}^{\prime}$ that was at $\mathrm{P}$ prior to deformation \\
\hline s & State vector \\
\hline $\mathrm{T}$ & $2 \times 2$ coordinate transformation matrix (eq. $(6 \mathrm{c}))$ \\
\hline $\mathrm{T}_{\text {aug }}$ & Augmented form of $\mathrm{T}$ for all degrees of freedom in a vector \\
\hline$T_{\mathrm{s}}$ & Duration \\
\hline$u, v$ & Deflections of $\mathrm{P}$ in $U V$ frame (Fig. 1,2) \\
\hline $\mathrm{u}(t)$ & $\begin{array}{l}3 N \times 1 \text { vector containing the instantaneous absolute coordinates of a subset of } \\
\text { FE nodes } Q_{n}(n=1 \ldots N)\end{array}$ \\
\hline $\mathrm{u}^{(\mathrm{rgd})}(t)$ & The absolute blade tip positions due to rotation of the blisk as a rigid body \\
\hline$\Delta I_{\mathrm{P}_{k}}$ & The moment of inertia of the segment about the nominal axis of rotation $\mathrm{OZ}$ \\
\hline$\Delta m_{\mathrm{P}_{k}}$ & The mass of the segment with mass centre at $\mathrm{P}_{k}$ (Fig. 2) \\
\hline$\Delta \mathrm{u}^{(\mathrm{def})}(t)$ & Dynamic response to excitation (eq. (1)) \\
\hline$\Delta \mathrm{u}^{(\mathrm{off})}(t)$ & Prescribed steady shift \\
\hline$X, Y$ & Coordinates in rotating frame (Fig. 1, 2) \\
\hline $\begin{array}{l}X^{(\mathrm{in})} \\
Y^{(\mathrm{in})}\end{array}$ & Coordinates in inertial frame (Fig. 1, 2) \\
\hline$Z$ & Coordinate along axis of rotation \\
\hline$X_{0}, Y_{0}, Z_{0}$ & Coordinates of undeformed position in frame $X Y Z$ (Fig. 1) \\
\hline$x, y, z$ & Local deformations in a rotating frame \\
\hline$\varphi(t)$ & Rigid rotation of blisk \\
\hline$\zeta_{r}$ & Modal damping ratio \\
\hline$\vartheta, \vartheta_{\mathrm{P}_{k}}$ & Angular coordinate of $\mathrm{P}, \mathrm{P}_{k}$ in rotating frame $X Y$ (Fig. 1, 2) \\
\hline$\theta_{x}, \theta_{y}, \theta_{z}$ & $\begin{array}{l}\text { The angular deformations about the respective local Cartesian axes, of a vector } n \\
\text { that is attached, and always perpendicular to, a local plane at the undeformed } \\
\text { position of point } \mathrm{P}\end{array}$ \\
\hline$\lambda$ & Eigenvalue \\
\hline$\Omega$ & Angular velocity \\
\hline$\Omega_{\mathrm{i}}, \Omega_{\mathrm{f}}$ & Initial and final angular velocities \\
\hline
\end{tabular}




$\begin{array}{ll}\omega & \text { Excitation frequency } \\ \varpi_{r} & \text { Undamped natural circular frequencies at } \Omega=0, r=1 \ldots H\end{array}$

\section{REFERENCES}

[1] C.-H. Yang, S.-C. Huang, Coupling Vibrations in Rotating Shaft-Disk-Blades System, Journal of Vibration and Acoustics. 129 (2007) 48-57.

[2] H. Ma, Y. Lu, Z. Wu, X. Tai, H. Li, B. Wen, A new dynamic model of rotor-blade systems, Journal of Sound and Vibration. 357 (2015) 168-194.

[3] I.Y. Shen, C.-P.R. Ku, A Nonclassical Vibration Analysis of a Multiple Rotating Disk and Spindle Assembly, Journal of Applied Mechanics. 64 (1997) 165-174.

[4] V. Omprakash, V. Ramamurti, Natural frequencies of bladed disks by a combined cyclic symmetry and Rayleigh-Ritz method, Journal of Sound and Vibration. 125 (1988) 357-366.

[5] T. Yokoyama, Free vibration characteristics of rotating Timoshenko beams, International Journal of Mechanical Sciences. 30 (1988) 743-755.

[6] S.Y. Lee, S.M. Lin, Bending Vibrations of Rotating Nonuniform Timoshenko Beams With an Elastically Restrained Root, Journal of Applied Mechanics. 61 (1994) 949955.

[7] H. Du, M.K. Lim, K.M. Liew, A Power Series Solution For Vibration Of A Rotating Timoshenko Beam, Journal of Sound and Vibration. 175 (1994) 505-523.

[8] V.T. Nagaraj, Approximate formula for the frequencies of a rotating Timoshenko beam, Journal of Aircraft. 33 (1996) 637-639.

[9] K.B. Subrahmanyam, K.R. V. Kaza, Vibration and Buckling of Rotating, Pretwisted, Preconed Beams Including Coriolis Effects, Journal of Vibration Acoustics Stress and Reliability in Design. 108 (1986) 140-149.

[10] H.H. Yoo, S.H. Shin, Vibration Analysis of Rotating Cantilever beams, Journal of Sound and Vibration. 212 (1998) 807-828.

[11] S.C. LIN, K.M. HSIAO, Vibration Analysis of A Rotating Timoshenko Beam, Journal of Sound and Vibration. 240 (2001) 303-322.

[12] J. a Dopkin, T.E. Shoup, Rotor Resonant Speed Reduction Caused by Flexibility of Disks, Journal of Engineering for Industry. 96 (1974) 1328-1333.

[13] F. Kushner, Disc Vibration - Rotating Blade and Stationary Vane Interaction, Journal of Mechanical Design. 102 (1980) 579-584. 
[14] D.R. Chivens, H.D. Nelson, The Natural Frequencies and Critical Speeds of a Rotating, Flexible Shaft-Disk System, Journal of Engineering for Industry. 97 (1975) 881-886.

[15] G. Jacquet-Richardet, G. Ferraris, P. Rieutord, Frequencies and Modes of rotating flexible Bladed Disk-Shaft Assemblies: A Global Cyclic Symmetry Approach, Journal of Sound and Vibration. 191 (1996) 901-915.

[16] E.F. Crawley, E.H. Ducharme, D.R. Mokadam, Analytical and Experimental Investigation of the Coupled Bladed Disk/Shaft Whirl of a Cantilevered Turbofan, Journal of Engineering for Gas Turbines and Power. 108 (1986) 567.

[17] A.A.S. Shahab, J. Thomas, Coupling effects of disc flexibility on the dynamic behaviour of multi disc-shaft systems, Journal of Sound and Vibration. 114 (1987) 435-452.

[18] S.-B. Chun, C.-W. Lee, Vibration Analysis of Shaft-Bladed Disk System by Using Substructure Synthesis and Assumed Modes Method, Journal of Sound and Vibration. 189 (1996) 587-608.

[19] R. Venkatachalam, S.B. Prabu, Vibration characteristics of orthotropic shaft-disk system with different constrained damping layers: experimental and numerical study, The International Journal of Advanced Manufacturing Technology. 65 (2013) 601610.

[20] Y.-Q. Yin, B. Zhang, Y. Li, W.-Z. Lu, Effect of Dead Load on Dynamic Characteristics of Rotating Timoshenko Beams, Mathematical Problems in Engineering. 2015 (2015) 1-10.

[21] H. Ma, F. Yin, Z. Wu, X. Tai, B. Wen, Nonlinear vibration response analysis of a rotor-blade system with blade-tip rubbing, Nonlinear Dynamics. 84 (2016) 1225-1258.

[22] Y.-J. Chiu, X.-Y. Li, Y.-C. Chen, S.-R. Jian, C.-H. Yang, I.-H. Lin, Three methods for studying coupled vibration in a multi flexible disk rotor system, Journal of Mechanical Science and Technology. 31 (2017) 5219-5229.

[23] R.L. Eshleman, R.A. Eubanks, On the Critical Speeds of a Continuous Shaft-Disk System, Journal of Engineering for Industry. 89 (1967) 645-652.

[24] M. Sakata, K. Kimura, S.K. Park, H. Ohnabe, Vibration of bladed flexible rotor due to gyroscopic moment, Journal of Sound and Vibration. 131 (1989) 417-430.

[25] M. Shahgholi, S.E. Khadem, S. Bab, Nonlinear vibration analysis of a spinning shaft with multi-disks, Meccanica. 50 (2015) 2293-2307.

[26] V. Ruffini, C.W. Schwingshackl, J.S. Green, Prediction Capabilities of Coriolis and Gyroscopic Effects in Current Finite Element Software, in: P. Pennacchi (Ed.), 
Proceedings of the 9th IFToMM International Conference on Rotor Dynamics, Springer, Cham, 2015: pp. 1853-1862.

[27] K. a Ansari, On the Importance of Shear Deformation, Rotatory Inertia, and Coriolis Forces in Turbine Blade Vibrations, Journal of Engineering for Gas Turbines and Power. 108 (1986) 319-324.

[28] R.M. Krupka, A.M. Baumanis, Bending-Bending Mode of a Rotating Tapered-Twisted Turbomachine Blade Including Rotatory Inertia and Shear Deformation, Journal of Engineering for Industry. 91 (1969) 1017-1024.

[29] F. Sisto, A. Chang, M. Sutcu, The Influence of Coriolis Forces on Gyroscopic Motion of Spinning Blades, Journal of Engineering for Power. 105 (1983) 342-347.

[30] N. Khader, R.G. Loewy, Shaft flexibility effects on the forced response of a bladeddisk assembly, Journal of Sound and Vibration. 139 (1990) 469-485.

[31] J. Xin, J. Wang, Investigation of Coriolis Effect on Vibration Characteristics of a Realistic Mistuned Bladed Disk, in: ASME. Turbo Expo: Power for Land, Sea, and Air, Volume 6: Structures and Dynamics, Parts A and B, Vancouver, British Columbia, Canada, 2011: pp. 993-1005.

[32] V. Ruffini, C. Schwingshackl, J. Green, Experimental and Analytical Study of Coriolis Effects in Bladed Disk, in: ASME. International Design Engineering Technical Conferences and Computers and Information in Engineering Conference, Volume 8: 27th Conference on Mechanical Vibration and Noise, Boston, Massachusetts, USA, 2015: p. V008T13A070.

[33] X. Kan, Z. Xu, B. Zhao, J. Zhong, Effect of coriolis force on forced response magnification of intentionally mistuned bladed disk, Journal of Sound and Vibration. 399 (2017) 124-136.

[34] J.S. Przemieniecki, Theory of Matrix Structural Analysis., McGraw-Hill, New York, 1985.

[35] V. Ramamurti, R. Kielb, Natural frequencies of twisted rotating plates, Journal of Sound and Vibration. 97 (1984) 429-449.

[36] J.R. Banerjee, Free Vibration of Centrifugally Stiffened Uniform and Tapered Beams Using the Dynamic Stiffness Method, Journal of Sound and Vibration. 233 (2000) $857-875$.

[37] J.R. Banerjee, Dynamic Stiffness Formulation and Free Vibration Analysis of Centrifugally Stiffened Timoshenko Beams, Journal of Sound and Vibration. 247 (2001) 97-115.

[38] J.R. Banerjee, Dynamic stiffness formulation for structural elements: A general approach, Computers \& Structures. 63 (1997) 101-103. 
[39] Y. Chen, J. Zhang, H. Zhang, X. Li, J. Zhou, Extraction of Natural Frequencies and Mode Shapes of Rotating Beams by Variational Iteration Method, International Journal of Structural Stability and Dynamics. 16 (2016) 1450106.

[40] P. Kohnke, Theory Reference for the Mechanical APDL and Mechanical Applications, 12th ed., ANSYS, Inc., Canonsburg, PA, USA, 2009.

[41] M. Mohamed, P. Bonello, P. Russhard, An Experimentally Validated Modal Model Simulator for the Assessment of Different Blade Tip Timing (BTT) Algorithms, Mechanical Systems and Signal Processing. (Under rev (n.d.).

[42] M. Mohamed, P. Bonello, P. Russhard, The Determination of Steady-State Movements Using Blade Tip Timing Data, in: ASME. Turbo Expo: Power for Land, Sea, and Air, Volume 7C: Structures and Dynamics, ASME, Oslo, Norway, 2018: p. V07CT35A010.

[43] F. Figaschewsky, B. Hanschke, A. Kühhorn, Efficient Generation of Engine Representative Tip Timing Data Based on a Reduced Order Model for Bladed Rotors, Journal of Engineering for Gas Turbines and Power. 141 (2018) 012503.

[44] I.F. Santos, C.M. Saracho, J.T. Smith, J. Eiland, Contribution to experimental validation of linear and non-linear dynamic models for representing rotor-blade parametric coupled vibrations, Journal of Sound and Vibration. 271 (2004) 883-904. doi:10.1016/S0022-460X(03)00758-2.

[45] M. Mohamed, P. Bonello, P. Russhard, A novel method for the determination of the change in blade tip timing probe sensing position due to steady movements, Mechanical Systems and Signal Processing. 126 (2019) 686-710.

[46] Ordinary Differential Equations, MATLAB R2019a. (2019). https://uk.mathworks.com/help/matlab/ordinary-differentialequations.html?s_tid=CRUX_lftnav (accessed July 1, 2019).

[47] F.J. Shaker, Effect of Axial Load on Mode Shapes and Frequencies of Beams, in: NASA Technical Note, NASA TN D-8109, Lewis Research Center, 1975.

[48] W. Zhang, W. Wang, H. Wang, J. Tang, Analytical and experimental investigation of bladed-disk/shaft coupled system, in: Proceedings of the Fourth International Conference on Rotor Dynamics, 1994: pp. 63-68.

[49] R.D. Blevins, Formulas for natural frequency and mode shape, Van Nostrand Reinhold, New York; 1979.

[50] R.C. Hibbeler, Mechanics of materials, 7th ed., Pearson Prentice Hall, Upper Saddle River, N.J, 2008. 
[51] J. Strnad, J. Liska, Diagnostic methods of a bladed disc mode shape evaluation used for shrouded blades in steam turbines, Journal of Physics: Conference Series. 659 (2015) 012051. 